\title{
PRECISE MEASUREMENT OF
}

\author{
$D^{0}, D^{ \pm}$and $D_{s}^{ \pm}$ \\ b.s $\%$ \\ MESON LIFETIMES
}

\author{
A Dissertation \\ Submitted to the Graduate School \\ of the University of Notre Dame \\ in Partial Fulfillment of the Requirements \\ for the Degree of \\ Doctor of Philosophy \\ by
}

Danilo L. Pušeljić

$\frac{\text { Pandal P. Rucht }}{\text { Randal C. Ruchti, Director }}$

Department of Physics

Notre Dame, Indiana

April, 1993

AAC4767 



\section{PRECISE MEASUREMENT OF \\ $D^{0}, D^{ \pm}$and $D_{s}^{ \pm}$ \\ MESON LIFETIMES}

Abstract

by

\section{Danilo Lj. Pušeljić}

The measurements of the $D^{0}, D^{ \pm}$and $D_{s}^{ \pm}$meson lifetimes are described. The $D^{0}$ meson has been observed through its decay into $K^{\mp} \pi^{ \pm}$and $K^{\mp} \pi^{ \pm} \pi^{\mp} \pi^{ \pm}$, the $D^{ \pm}$meson through its decay into $K^{\mp} \pi^{ \pm} \pi^{ \pm}$, and the $D_{s}^{ \pm}$meson through its decay into $\phi^{0} \pi^{ \pm}$. The data sample was obtained in a high energy photoproduction experiment E687 at the Fermilab Wide Band Laboratory during the 1990-91 fixed target running period with a mean tagged photon energy of $220 \mathrm{GeV}$. The final lifetime samples consisted of $\approx 17500 D^{0}, \approx 11500 D^{ \pm}$, and $\approx 900 D_{s}^{ \pm}$fully reconstructed decays. The lifetimes were obtained by performing a binned maximum likelihood fit to the observed proper time distributions. The measured lifetimes of the $D^{0}, D^{ \pm}$and $D_{s}^{ \pm}$are $0.4200 \pm 0.0045 \pm 0.0030,1.042 \pm 0.015 \pm 0.011$, and $0.475 \pm 0.020 \pm 0.007$ picoseconds respectively. (The first error is statistical error while the second is systematic error.) 

To my father

Ljubisav M. Pušeljić 



\section{TABLE OF CONTENTS}

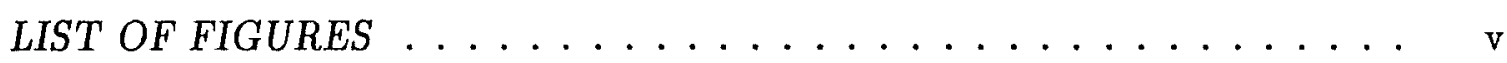

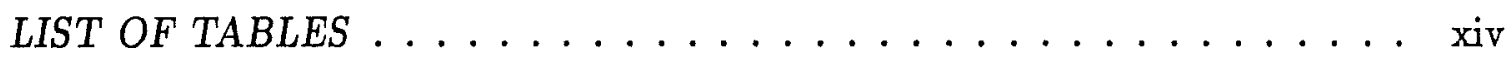

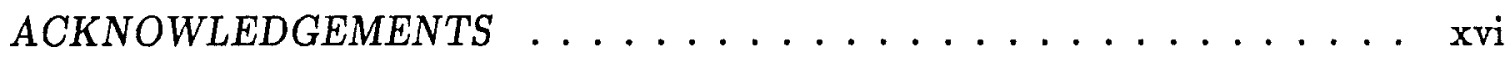

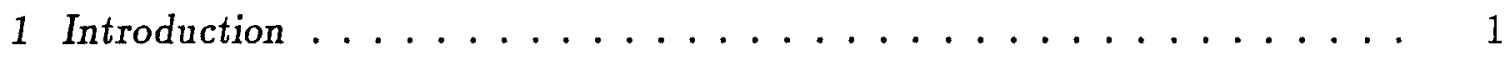

1.1 Weak Decays of Heavy Quarks . . . . . . . . . . . . 1

1.1.1 Weak Decays of Charm Mesons . . . . . . . . . . 6

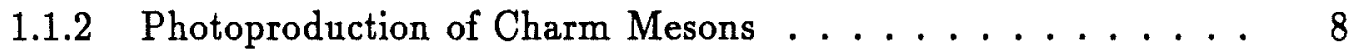

1.2 Scope of the Thesis $\ldots \ldots \ldots \ldots \ldots \ldots \ldots \ldots$

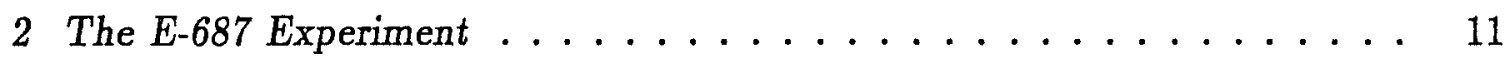

2.1 Photon Beam . . . . . . . . . . . . . . 11

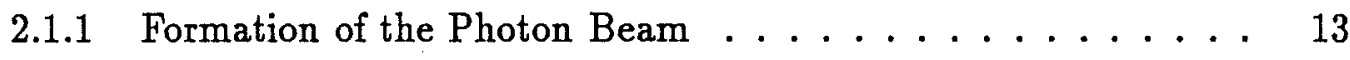

2.1.2 Energy determination of the Photon Beam . . . . . . . 16

2.2 Spectrometer $\ldots \ldots \ldots \ldots \ldots \ldots \ldots \ldots \ldots \ldots$

2.2 .1 Experimental Target $\ldots \ldots \ldots \ldots \ldots \ldots \ldots$

2.2.2 Charged Particle Tracking . . . . . . . . . . . 23

2.2 .3 Čerenkov system $\ldots \ldots \ldots \ldots \ldots \ldots \ldots$

2.2.4 Electromagnetic Calorimetry . . . . . . . . . . . 29

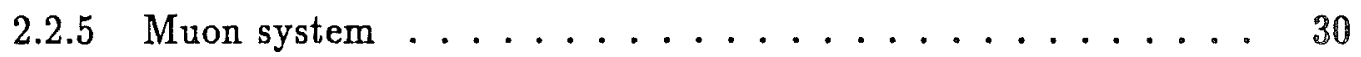

2.2 .6 Hadronic Calorimetry . . . . . . . . . . . . . . . 30

2.2 .7 Trigger $\ldots \ldots \ldots \ldots \ldots \ldots \ldots \ldots \ldots \ldots$

2.2 .8 Data Acquisition $\ldots \ldots \ldots \ldots \ldots \ldots \ldots \ldots$

3 Data Analysis ........................ 34

3.1 Data Reconstruction . . . . . . . . . . . . 34

3.1.1 Charged Particle Tracking . . . . . . . . . . . 35

3.1.2 Momentum Determination . . . . . . . . . . . . 40 
3.1.3 Particle Identification by the Čerenkov system . . . . . . 42

3.2 Data Reduction . . . . . . . . . . . . . . . . 48

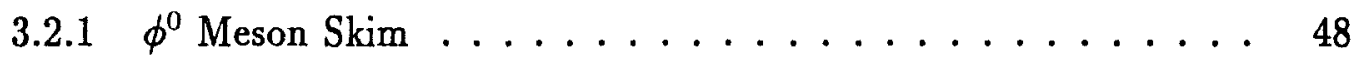

$3.2 .2 \quad D$ Meson Skim $\ldots \ldots \ldots \ldots \ldots \ldots \ldots \ldots$

3.3 The Final Event Selection $\ldots \ldots \ldots \ldots \ldots \ldots \ldots \ldots$

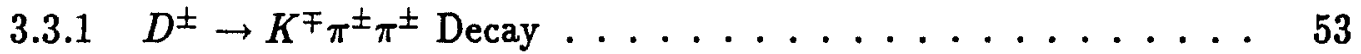

3.3.2 $\quad D^{0} \rightarrow K \pi$ and $K \pi \pi \pi$ Decays $\ldots \ldots \ldots \ldots \ldots \ldots$

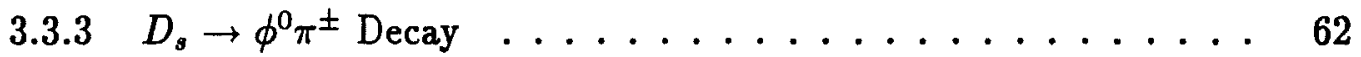

4 Charm Meson Lifetime Analysis . . . . . . . . . . . . . 66

4.1 Lifetime Measurement Technique . . . . . . . . . . 66

4.1.1 The Likelihood Function . . . . . . . . . . . 67

4.2 Sources of Systematic Uncertainty . . . . . . . . . . . . 73

4.2.1 The $f\left(t^{\prime}\right)$ Correction Function . . . . . . . . . . 74

4.2.2 Uncertainty in the Photon Beam Profile $\ldots \ldots \ldots \ldots$

4.2.3 Uncertainty in the $D$ Momentum Spectrum $\ldots \ldots \ldots \ldots$

4.2.4 Uncertainty in the Absorption Correction . . . . . . . . 78

4.2.5 Uncertainty in the Background Modelling . . . . . . 81

$4.3 \quad D^{ \pm}$Lifetime . . . . . . . . . . . . . . . . . . . 82

$4.4 D^{0}$ Lifetime $\ldots \ldots \ldots \ldots \ldots \ldots \ldots \ldots$

$4.5 D_{s}^{ \pm}$Lifetime $\ldots \ldots \ldots \ldots \ldots \ldots \ldots \ldots \ldots \ldots \ldots \ldots$

5 Summary and Conclusions . . . . . . . . . . . . . . 112

5.1 Lifetime Measurements . . . . . . . . . . . . . . . . . 112

5.2 Final Comments $\ldots \ldots \ldots \ldots \ldots \ldots \ldots \ldots \ldots$

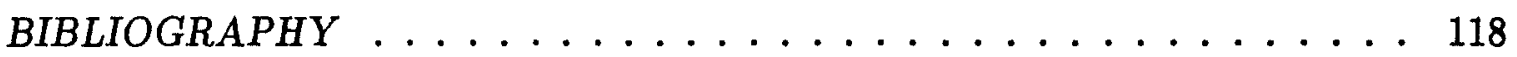




\section{LIST OF FIGURES}

1.1 Heavy Quark Decay Diagram $\ldots \ldots \ldots \ldots \ldots \ldots$

1.2 Qualitative picture of the weak decay of a heavy meson into five hadrons. Thick line represents the heavy quark, wavy lines the gluons, black dot the weak interaction, and dashed circle the boundary of the confine-

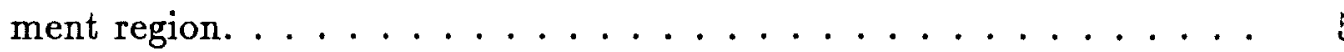

1.3 Lowest Order Diagrams for Charm Decays: a) external W-emission; b) internal $\mathrm{W}$-emission; c) $\mathrm{W}$-exchange; d) $\mathrm{W}$-annihilation . . . . . 7

1.4 a) Photon-gluon fusion process; b) Bethe-Heitler process. . . . . . . 9

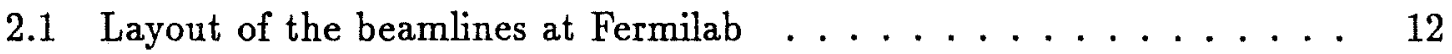

2.2 The $\mathrm{E}-687$ beam line layout $\ldots \ldots \ldots \ldots \ldots \ldots \ldots$

2.3 The Recoil Electron Shower Detector (RESH) layout $\ldots \ldots \ldots \ldots$

2.4 Tagged Photon Spectrum $\ldots \ldots \ldots \ldots \ldots \ldots$

2.5 The Layout of the E-687 detector system $\ldots \ldots \ldots \ldots \ldots$

2.6 The layout of the silicon microstrip detector $\ldots \ldots \ldots \ldots$

2.7 Plan view of the three Cerenkov counters: a) C1; b) C2; c) C3. . . . 28

3.1 Linking efficiency of kaons as a function of momentum for: a) 3-station tracks; b) 5 -station tracks. . . . . . . . . . . . . . 41

3.2 Momentum ranges where certain identification classes are possible: a) 3 -station tracks; b) 5 -station tracks. . . . . . . . . . . 45 
3.3 Kaon ID efficiency as a function of momentum for: a) 3-station tracks;

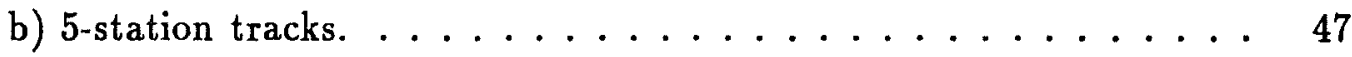

$3.4 K^{+} K^{-}$invariant mass distribution $\ldots \ldots \ldots \ldots \ldots \ldots$

3.5 $K \pi \pi$ invariant mass distribution for various $\ell / \sigma_{\ell}$ requirements: a) $\ell / \sigma_{\ell}>$ 5 ; b) $\ell / \sigma_{\ell}>8$; c) $\ell / \sigma_{\ell}>11$; d) $\ell / \sigma_{\ell}>14$; e) $\ell / \sigma_{\ell}>17$; f) $\ell / \sigma_{\ell}>19$.

3.6 The invariant mass distribution of the $K \pi$ TAG sample for various $\ell / \sigma_{\ell}$ requirements: a) $\ell / \sigma_{\ell}>0$; b) $\ell / \sigma_{\ell}>3$; c) $\ell / \sigma_{\ell}>6$; d) $\ell / \sigma_{\ell}>9$; e) $\left.\ell / \sigma_{\ell}>12 ; f\right) \ell / \sigma_{\ell}>15 \ldots \ldots \ldots \ldots \ldots \ldots$

3.7 The invariant mass distribution of the $K \pi \pi \pi$ TAG sample for various $\ell / \sigma_{\ell}$ requirements: a) $\ell / \sigma_{\ell}>2$; b) $\ell / \sigma_{\ell}>5$; c) $\ell / \sigma_{\ell}>8$; d) $\ell / \sigma_{\ell}>11$; e) $\ell / \sigma_{\ell}>14 ;$ f) $\ell / \sigma_{\ell}>17 \ldots \ldots \ldots \ldots \ldots$

3.8 The invariant mass distribution of the $K \pi \pi \pi$ NOTAG sample for various $\ell / \sigma_{\ell}$ requirements: a) $\ell / \sigma_{\ell}>7$; b) $\ell / \sigma_{\ell}>9$; c) $\ell / \sigma_{\ell}>11$; d) $\ell / \sigma_{\ell}>13$; e) $\ell / \sigma_{\ell}>15$; f) $\ell / \sigma_{\ell}>17 \ldots \ldots \ldots \ldots$

3.9 The $\cos \theta$ distributions for the $\phi^{0} \pi^{ \pm}$decay: a) Monte Carlo; b) $D_{s}^{ \pm}$ signal region; c) mass regions outside the signal region. . . . . . . .

3.10 The invariant mass distribution of the $\phi^{0} \pi^{ \pm}$sample for various $\ell / \sigma_{\ell}$ requirements: a) $\ell / \sigma_{\ell}>3$; b) $\ell / \sigma_{\ell}>8$; c) $\ell / \sigma_{\ell}>13$. . . . . .

4.1 Error in proper time as a function of proper time $\ldots \ldots \ldots \ldots$

4.2 Distribution of the differences between input and reconstructed proper times for Monte Carlo. Overlayed on top is a result of a fit with a sum of a Gaussian and an exponential function. . . . . . . . . . . 
4.3 Uncertainty of the fitted lifetime caused by finite Monte Carlo statistic for $f\left(t^{\prime}\right)$ generated with: a) $400 \mathrm{~K} D^{0} \rightarrow K \pi$ TAG M.C. events; b) $400 \mathrm{~K}$ $D^{0} \rightarrow K \pi \pi \pi$ TAG M.C. events; c) $400 \mathrm{~K} D^{0} \rightarrow K \pi \pi \pi$ NOTAG M.C. events; d) $1.75 \mathrm{M} D^{ \pm} \rightarrow K^{\mp} \pi^{ \pm} \pi^{ \pm}$M.C. events; e) $500 \mathrm{~K} D_{s}^{ \pm} \rightarrow \phi^{0} \pi^{ \pm}$ M.C. events. . . . . . . . . . . . . . . . .

4.4 Transverse beam profile in the $\mathrm{x}$ direction in data and Monte Carlo for: a) $D^{0} \mathrm{X}$-profile; b) $D^{0} \mathrm{Y}$-profile; c) $D^{ \pm} \mathrm{X}$-profile; d) $D^{ \pm} \mathrm{Y}$-profile; solid and dashed lines represent data and Monte Carlo distributions respectively. . . . . . . . . . . . . . . .

4.5 Comparison of $D$ momentum distributions in data and Monte Carlo for; a) $D^{0}$ sample; b) $D^{ \pm}$sample; solid and dashed histograms represent data and Monte Carlo distributions respectively. . . . . . . . . .

4.6 Effect of the hadronic absorption of charm particles and their secondaries as a function of proper time for; a) $D^{0} \rightarrow K \pi$; b) $D^{0} \rightarrow K \pi \pi \pi$; c) $D^{ \pm} \rightarrow K^{\mp} \pi^{ \pm} \pi^{ \pm}$; d) $D_{s}^{ \pm} \rightarrow \phi^{0} \pi^{ \pm}$. Dotted, dashed and solid lines represent effects of charm particle, daughter particle, and both absorp-

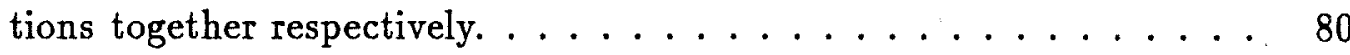

4.7 The fitted lifetime as a function of an $\ell / \sigma_{\ell}$ cut used for: a) Monte Carlo with $f\left(t^{\prime}\right)=1$; b) data with $f\left(t^{\prime}\right)=1$; c) Monte Carlo with the standard $f\left(t^{\prime}\right)$; d) data with the standard $f\left(t^{\prime}\right)$. Dash-dotted line represents the current world average value of the $D^{ \pm}$lifetime (1.066 \pm $0.023 \mathrm{ps})$. The dotted lines represent the current error on the average value. 
4.8 The background subtracted and Monte Carlo corrected lifetime evolution of the $D^{ \pm}$sample for $\ell / \sigma_{\ell}>15$ shown as solid line histogram. The crosses and dots represent the utilized reduced proper time distributions for the signal and sideband regions respectively. An exponential function with a fitted lifetime is overlayed. . . . . . . . . .

4.9 The correction factor $f\left(t^{\prime}\right)$ to the reduced proper time distribution of the $D^{ \pm}$decays as a function of reduced proper time. . . . . . .

4.10 The result of simulated "mini-experiments": a) Distribution of fitted lifetimes; b) Distribution of fitted lifetimes minus the input lifetime divided by its error. . . . . . . . . . . . .

4.11 The $D^{ \pm}$fitted lifetime as a function of the $\ell / \sigma_{\ell}$ cut used for Monte Carlo utilizing the standard $f\left(t^{\prime}\right)$ for different momentum regions: a) momentum $P<80 \mathrm{GeV} / \mathrm{c}$; b) momentum $80<P<120 \mathrm{GeV} / \mathrm{c}$; c) momentum $P>120 \mathrm{GeV} / \mathrm{c}$. Dashed-dotted line represents the Monte Carlo input value for the $D^{ \pm}$lifetime. . . . . . . . .

4.12 The $D^{ \pm}$fitted lifetime as a function of an $\ell / \sigma_{\ell}$ cut for Monte Carlo utilizing the standard $f\left(t^{\prime}\right)$ for different radial regions: a) inner Radius $R<0.5 \mathrm{~cm}$; b) middle Radius $0.5<R<1.0 \mathrm{~cm}$; ) outer Radius

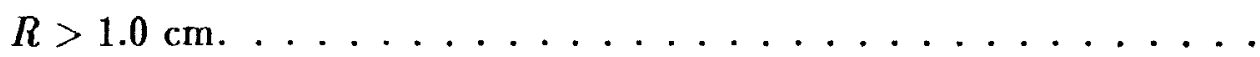

4.13 The $D^{ \pm}$fitted lifetime as a function of an $\ell / \sigma_{\ell}$ cut used for Monte Carlo utilizing the standard $f\left(t^{\prime}\right)$ for different fractions of the sideband regions: a) low side band $100 \%$ (solid line top most); b) low side band $75 \%$ (dashed line); c) low side band 50\% (dotted line); d) low side band $25 \%$ (dashed-dotted line); e) low side band $0 \%$ (solid line bottom most). 90 
4.14 A comparison of measured $D^{ \pm}$lifetimes from different consistency checks and studies of systematic effects. The values are the results obtained: a) in this analysis; b) using $0 \%$ of low sideband; c) using $100 \%$ of low sideband; d) using upstream primary vertices only; e) using downstream primary vertices only; f) using $D^{+}$(particle) events only; g) using $D^{-}$(anti-particle) events only; h) using the 1990 data only; i) using the 1991 data only; j) using only decays that have occurred in the vacuum; $k$ ) PCL $>10 \%$, and DCL $>10 \%$ sample. . . .

4.15 The fitted lifetime as a function of $\ell / \sigma_{\ell}$ for $:$ a) $K \pi$ TAG sample with $f\left(t^{\prime}\right)=1$; b) $K \pi$ TAG sample with standard $f\left(t^{\prime}\right) \ldots \ldots \ldots \ldots$

4.16 The fitted lifetime as a function of $\ell / \sigma_{\ell}$ for: a) $K \pi \pi \pi$ TAG sample with $f\left(t^{\prime}\right)=1$; b) $K \pi \pi \pi$ TAG sample with standard $f\left(t^{\prime}\right)$; c) $K \pi \pi \pi$ NOTAG sample with $f\left(t^{\prime}\right)=1$; d) $K \pi \pi \pi$ NOTAG sample with standard $f\left(t^{\prime}\right)$;

4.17 The $K \pi$ TAG sample lifetime as a function of $\ell / \sigma_{\ell}$ cut for the different fractions of sideband regions: a) low side band $100 \%$; b) low side band $75 \%$; c) low side band $50 \%$; d) low side band $25 \%$; e) low side band $0 \% .96$

4.18 The $K \pi \pi \pi$ TAG sample lifetime as a function of $\ell / \sigma_{\ell}$ cut for the different fractions of sideband regions: a) low side band 100\%; b) low side band $75 \%$; c) low side band $50 \%$; d) low side band $25 \%$; e) low side

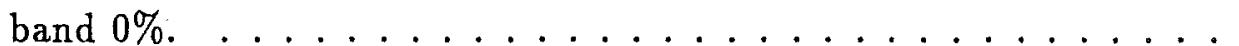

4.19 The $K \pi \pi \pi$ NOTAG sample lifetime as a function of $\ell / \sigma_{\ell}$ cut for the different fractions of sideband regions: a) low side band $100 \%$; b) low side band $75 \%$; c) low side band $50 \%$; d) low side band $25 \%$; e) low side band $0 \% \ldots \ldots \ldots \ldots \ldots \ldots \ldots \ldots \ldots \ldots$ 
4.20 a) The background subtracted and $f\left(t^{\prime}\right)$ corrected reduced proper time distribution of the $K \pi$ TAG sample (shown as a solid histogram) for $\ell / \sigma_{\ell}>3$; crosses and dotted lines represent the signal and sideband reduced proper time distributions respectively. Superimposed is an exponential function with the corresponding fitted lifetime. b) $f\left(t^{\prime}\right)$

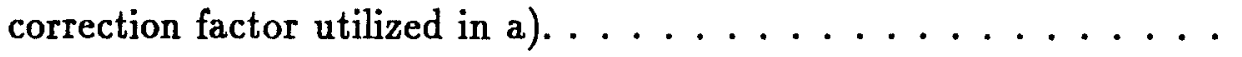

4.21 a) The background subtracted and $f\left(t^{\prime}\right)$ corrected reduced proper time distribution of the $K \pi \pi \pi$ TAG sample (shown as a solid histogram) for $\ell / \sigma_{\ell}>2$; crosses and dotted lines represent the signal and sideband reduced proper time distributions respectively. Superimposed is an exponential function with the corresponding fitted lifetime. b) $f\left(t^{\prime}\right)$ correction factor utilized in a). ................ 10

4.22 a) The background subtracted and $f\left(t^{\prime}\right)$ corrected reduced proper time distribution of the $K \pi \pi \pi$ NOTAG sample (shown as a solid histogram) for $\ell / \sigma_{\ell}>7$; crosses and dotted lines represent the signal and sideband reduced proper time distributions respectively. Superimposed is an exponential function with the corresponding fitted lifetime. b) $f\left(t^{\prime}\right)$ correction factor utilized in a). . . . . . . . . . . . . 101

4.23 The result of simulated "mini-experiments" for the $K \pi$ TAG sample: a) distribution of the fitted lifetime for the $K \pi$ TAG sample; b) deviation of the fitted lifetime from the input value divided by its error for $K \pi$ TAG sample. . . . . . . . . . . . . . . 102 
4.24 The result of simulated "mini-experiments" for $K \pi \pi \pi$ TAG and NOTAG samples: a) distribution of fitted lifetimes for the $K \pi \pi \pi$ TAG sample; b) deviation of the fitted lifetime from the input value divided by its error for $K \pi \pi \pi$ TAG sample; c) distribution of fitted lifetimes for the $K \pi \pi \pi$ NOTAG sample; d) deviation of the fitted lifetime the input value divided by its error for $K \pi \pi \pi$ NOTAG sample. . . . . . 103

4.25 The lifetime of the $D_{s}^{ \pm}$as a function of $\ell / \sigma_{\ell}$ cut for: a) Monte Carlo sample with $f\left(t^{\prime}\right)=1$; b) data sample with $f\left(t^{\prime}\right)=1$; c) Monte Carlo sample with standard $f\left(t^{\prime}\right) ;$ d) data sample with standard $f\left(t^{\prime}\right) . \ldots$

4.26 The background subtracted and Monte Carlo corrected lifetime evolution of the $D_{s}^{ \pm}$sample for $\ell / \sigma_{\ell}>3$ shown as the solid-lined histogram. The crosses show the reduced proper time distribution of the events in the signal region and the dotted-lined histogram shows the side band proper time distribution. The straight line is an exponential with fitted

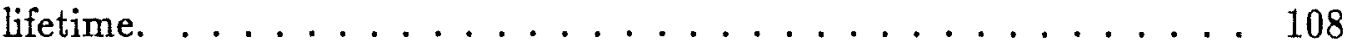

4.27 The correction factor $f\left(t^{\prime}\right)$ to the $D_{s}^{ \pm}$reduced proper time distribution as a function of reduced proper time for $\ell / \sigma_{\ell}>3 \ldots \ldots \ldots 108$

4.28 The results of fits to simulated "mini-experiments" for $D_{s}^{ \pm} \rightarrow \phi^{0} \pi^{ \pm}$ sample: a) distribution of fitted lifetimes; b) deviation of the fitted lifetime from input value divided by its error. . . . . . . . . . 109 
4.29 A comparison of measured $D_{s}$ lifetimes from different consistency checks and studies of systematic effects. The values are the results obtained: a) in this analysis; b) using side-band regions $1.788-1.808 \mathrm{GeV} / \mathrm{c}^{2}$ and $2.008-2.028 \mathrm{GeV} / \mathrm{c}^{2} ; \mathrm{c}$ ) using only side-band region $2.008-2.048$ $\left.\mathrm{GeV} / \mathrm{c}^{2} ; \mathrm{d}\right)$ eliminating events in the $D^{+}$reflection region; e) eliminating events in the $\Lambda_{c}$ reflection region; f) using only events with $D_{s}$ momentum $>95 \mathrm{GeV} / \mathrm{c} ; \mathrm{g})$ using only events with $D_{s}$ momentum $<95$ $\mathrm{GeV} / \mathrm{c} ; \mathrm{h}$ ) using $D_{s}^{+}$(particle) events only; i) using $D_{s}^{-}$(anti-particle) events only; j) using upstream primary vertices only, and $k$ ) using downstream primary vertices only. . . . . . . . . . 110

5.1 Comparison of the measured $D^{ \pm}$lifetime to other measurements used by the [Pdg 92] to calculate the previous worl average value shown as the horizontal dashed-dotted line. Vertical solid and dashed lines represent statistical and systematic errors respectively for a given measurement. Horizontal dotted line represents the error on the world average value. . . . . . . . . . . . . . . . . 114

5.2 Comparison of the measured $D^{0}$ lifetime to other measurements used by the [Pdg 92] to calculate the previous worl average value shown as the horizontal dashed-dotted line. Vertical solid and dashed lines represent statistical and systematic errors respectively for a given measurement. Horizontal dotted line represents the error on the world

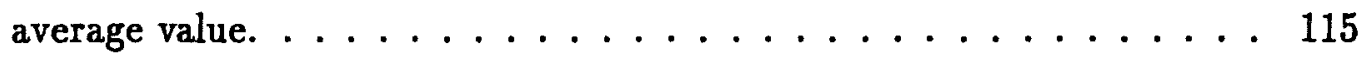


5.3 Comparison of the measured $D_{s}^{ \pm}$lifetime to other measurements used by the $[\mathrm{Pdg} 92]$ to calculate the previous worl average value shown as the horizontal dashed-dotted line. Vertical solid and dashed lines represent statistical and systematic errors respectively for a given measurement. Horizontal dotted line represents the error on the world

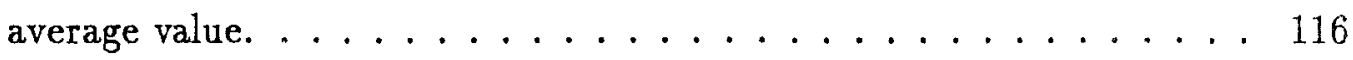




\section{LIST OF TABLES}

1.1 Masses and lifetimes of pseudoscalar charm mesons . . . . . . . 6

2.1 Wide Band beam properties ................. 14

2.2 Table with individual subsystem dimensions and their location . . . 21

2.3 Table with individual subsystem dimensions and their location . . . 22

2.4 Properties of the silicon microstrip detector ........... . 25

2.5 Analysis magnet specifications . . . . . . . . . . . . 26

2.6 Table with Čerenkov system properties . . . . . . . . . . . 27

3.1 Values assigned to Cerenkov ISTATP variable . . . . . . . . . 44

3.2 List of skimming strategies . . . . . . . . . . . . . . 49

$3.3 \quad D$ meson skim selection criteria $\ldots \ldots \ldots \ldots$

3.4 Cuts used to obtain the final $D^{ \pm} \rightarrow K^{\mp} \pi^{ \pm} \pi^{ \pm}$sample . . . . . . . 55

3.5 Cuts used to obtain the final $K \pi$ TAG sample . . . . . . . . . 56

3.6 Cuts used to obtain the final $D^{0} \rightarrow K \pi \pi \pi$ samples . . . . . . 58

3.7 Cuts used to obtain the final $D_{s}^{ \pm}$sample $\ldots \ldots \ldots 63$

4.1 The $D^{ \pm}$fit parameters $\ldots \ldots \ldots \ldots$. . . . . . . . . 87

4.2 Systematic uncertainties of the $D^{ \pm}$lifetime . . . . . . . . . 92

4.3 Mass regions of the $D^{0}$ samples $\ldots \ldots \ldots$. . . . . . . . . 93

4.4 Systematic uncertainties of the $D^{0}$ lifetime $\left(\times 10^{-12} \mathrm{sec}.\right) \ldots \ldots 2$

4.5 The lifetimes of the $D^{0}$ samples $\ldots \ldots \ldots$. . . . . . . . . . 102 
4.6 The $D_{s}^{ \pm}$fit parameters . . . . . . . . . . . 106

4.7 Systematic uncertainties of the $D_{s}^{ \pm}$lifetime . . . . . . . . 111

5.1 Summary of measured lifetimes ... . . . . . . . . 113

5.2 Comparison of measured lifetimes . . . . . . . . . 117 


\section{ACKNOWLEDGEMENTS}

The success of this experiment shows itself in the number of thesis and papers produced from it with many more to follow. This was made possible by relentless contributions of many members of the E-687 Collaboration for over a decade. I am very proud for being a member of such a gifted group of people much of whose work has made this thesis possible, a collective thanks to everyone in the collaboration.

I would like to especially thank my research director Randy Ruchti for his help and encouragement during all phases of my graduate studies and for being there as a friend. I'd also like to thank Neal Cason, Bill Shephard, John Losecco and Jim Bishop for asking the right questions and baring with me during long discussions.

Joel Butler, Jim Wiss, and John Cumalat provided a model of dedication and expertize, I thank them for their guidence, encouragement and numerous discussions. I would also like to thank Jerry Busenitz, Ray Culbertson, Harry Cheung, Daniele Pedrini, Pual Lebrun, Art Kreymer, Clara Castoldi and Carlo Dallapiccola from much of whose work and discussions I have benefitted from.

It has been a long haul, and the greatest gift from this time had been numerous friendships. To my office mates and most of all friends John Cunningham and Robert Gardner I thank for their perpetual encouragement, advice and friendship. The Kilbury family will always be close to my heart for their support and friendship over the years. Cash and Mary will always be our allies. My life has been enriched by friendships with Gino and Sanja Serpa, Zeid Ayer, Ray Mountain, and Tzufen (Tony) Lin. 
Finally, and most importantly many years as a graduate student would have been considerably harder without the care and support of my family. I am especially grateful to my wife Liana whose invaluable help and understanding was often taken for granted. Most of all I am indebted to my parents Olga and Ljubisav for their love, emotional and financial support over the years which laid the foundation of my education. I'd also like to thank my brothers Veselin and Miloš Čuljković who were always with me in the time of need. 


\section{CHAPTER 1}

\section{Introduction}

Weak decays have always been a rich source of information about the form and symmetry of the basic interactions as well as about the structure of the constituents of matter. They are essential for testing and determining the parameters of the standard $\mathrm{SU}(2)^{*} \mathrm{U}(1)$ model of electromagnetic and weak interactions. Many of its parameters are unmeasured directly or are only poorly known, like the mass of the top quark or the charged current couplings of the heavy quarks. It is, however, not straightforward to obtain any new information about the standard model from weak decays of heavy quarks since quarks are necessarily confined inside hadronic bound states by a strong force. The confinement mechanism is still not understood completely so the weak decays of heavy quarks thus also offer a chance of achieving a deeper insight into strong interaction dynamics.

\subsection{Weak Decays of Heavy Quarks}

In the standard model heavy quark weak decay proceeds through emission of a charged intermediate vector boson (Fig. 1.1). In this picture the Lagrangian for flavor changing interactions is given by [Ren 90$]$

$$
\mathcal{L}=\frac{g}{2 \sqrt{ } 2}\left\{\mathcal{J}^{\mu} \mathcal{W}_{\mu}^{+}+\text {h.c. }\right\}
$$


process of the hadron (it is just a spectator), all three charm mesons are expected to have the same lifetime. Table 1.1 shows that the lifetime of the $D^{+}$is about 2.5 times greater than the lifetime of the $D^{0}$. Originally it was hypothesized that the presence of annihilation/exchange diagrams in the $D^{0}$ and $D_{s}^{+}$decays was responsible for their shorter lifetimes, but it contradicted other arguments, such as the helicity suppression [Ros 80] of annihilation decays.

The currently favored theory [Bil 84] [Gub 79] bases the explanation on the fact that external and internal $\mathrm{W}$-emission, in the case of the $D^{ \pm}$produce identical final states which interfere destructively, hence lengthening its lifetime. The $D^{0}$ and $D_{s}^{+}$ decays on the other hand have different quarks in the final state and no interference occurs.

The next question that arises is whether $D_{s}^{+}$and $D^{0}$ lifetimes are equal or not, and if not what is the mechanism that produces the difference? At the level of current world statistics it is not clear whether the $D_{s}^{ \pm}$lifetime is somewhat longer or equal to the $D^{0}$ lifetime. A precise measurement of these lifetimes will assist in determining this ambiguity.

\subsubsection{Photoproduction of Charm Mesons}

The E-687 experiment utilizes a high energy photon beam to produce charm states in photon-nucleus interactions. A description of this process is given by the photongluon fusion model [Jon 78]. In this model the photon "fuses" with a gluon from the target to produce a quark-antiquark pair (a process very analogous to the BetheHeitler pair production). Fig.1.4 shows the lowest order QCD diagram responsible for this process and the analogous Bethe-Heitler pair production diagram.

Besides photoproduction, heavy quarks could be produced in $e^{+} e^{-}$collisions as well as in hadron-hadron or hadron-target collisions. The main advantage of a fixedtarget experiment over an $e^{+} e^{-}$collider experiment to measure the lifetimes of charm 


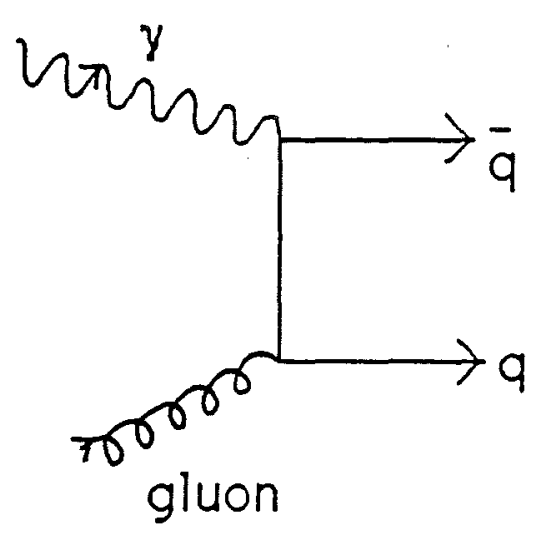

a)

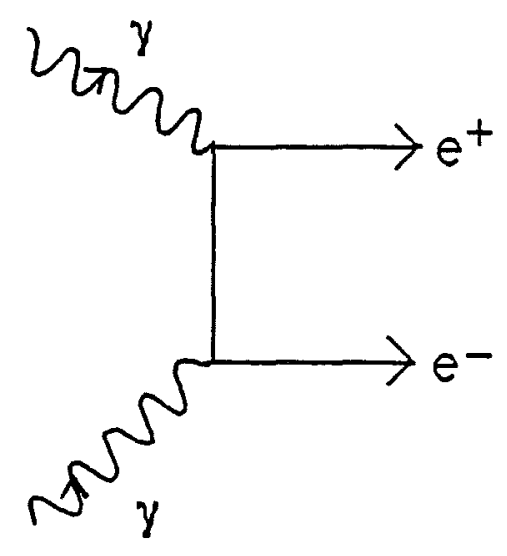

b)

Figure 1.4. a) Photon-gluon fusion process; b) Bethe-Heitler process.

states derives from the longer laboratory particle decay lengths produced by the Lorentz boost. In comparison to hadroproduction, photon-nucleus interactions produce charm particles more frequently (by a factor of 10). Hence the backgrounds are lower and signal extraction easier.

\subsection{Scope of the Thesis}

This thesis describes a high statistics measurement of the $D^{ \pm}, D^{0}$, and $D_{s}^{ \pm}$lifetimes. The data sample has been obtained by a fixed target, high energy photoproduction experiment E-687 at Fermilab ${ }^{1}$ The accumulated data sample utilized in lifetime measurements consists of $11500 D^{ \pm} \rightarrow K^{\mp} \pi^{ \pm} \pi^{ \pm}, 17300 D^{0} \rightarrow K^{-} \pi^{+}$and $D^{0} \rightarrow$ $K^{-} \pi^{+} \pi^{-} \pi^{+}$, and $900 D_{s}^{ \pm} \rightarrow \phi \pi^{ \pm}$decays. This sample triples the world's sample of $D^{ \pm}$and $D^{0}$ decays, and more than doubles the world $D_{s}^{ \pm}$meson sample. The details of the experiment are discussed in Chapter 2. Chapter 3 describes the data

\footnotetext{
${ }^{1}$ Fermi National Accelerator Laboratory, Batavia, Mlinois, U.S.A.
} 
Table 1.1. Masses and lifetimes of pseudoscalar charm mesons

\begin{tabular}{|c|c|c|}
\hline Meson & Mass $\left(\mathrm{MeV} / c^{2}\right)$ & Lifetime $\left(\times 10^{-13}\right.$ sec. $)$ \\
\hline$D^{+}$ & $1869.3 \pm 0.5$ & $10.66 \pm 0.23$ \\
$D^{0}$ & $1864.5 \pm 0.5$ & $4.20 \pm 0.08$ \\
$D_{s}^{+}$ & $1968.8 \pm 0.7$ & $4.50_{-0.26}^{+0.30}$ \\
\hline
\end{tabular}

\subsubsection{Weak Decays of Charm Mesons}

The charm mesons that decay weakly are the pseudoscalar isospin-doublet

$$
\left\{D^{+}=(c \bar{d}), \quad D^{0}=(c \bar{u})\right\}
$$

and the isospin-singlet

$$
D_{s}^{+}=(c \bar{s})
$$

All three states (and their charge conjugates) are established experimentally with a number of their properties measured to a resonable precision [Pdg 92]. The current world-average masses and lifetimes are displayed in Table 1.1. In the limit $\theta_{23}=\theta_{13}=$ 0 the third generation decouples and the CKM matrix given by Eq.1.4 reduces to the usual Cabibbo mixing of the first two generations with $\theta_{12}$ identified as the Cabibbo angle $\theta_{c}[\mathrm{Cab} 63]$. The value of $\theta_{c}$ has been measured by examining the weak decays of kaons and pions giving a value, $\theta_{c} \approx 13^{\circ}\left(\sin \theta_{c} \approx 0.22\right.$ and $\left.\cos \theta_{c} \approx 0.97\right)$. In this picture the coupling of the $W$ boson to the quarks is proportional either to $\cos \theta_{c}$ or $\sin \theta_{c}$ separating transitions into Cabibbo allowed and suppressed respectively. Fig.1.3 shows the lowest order diagrams present in charm decays. In the "spectator" approximation, where the non-charm quark is assumed not to influence the decay 
a)

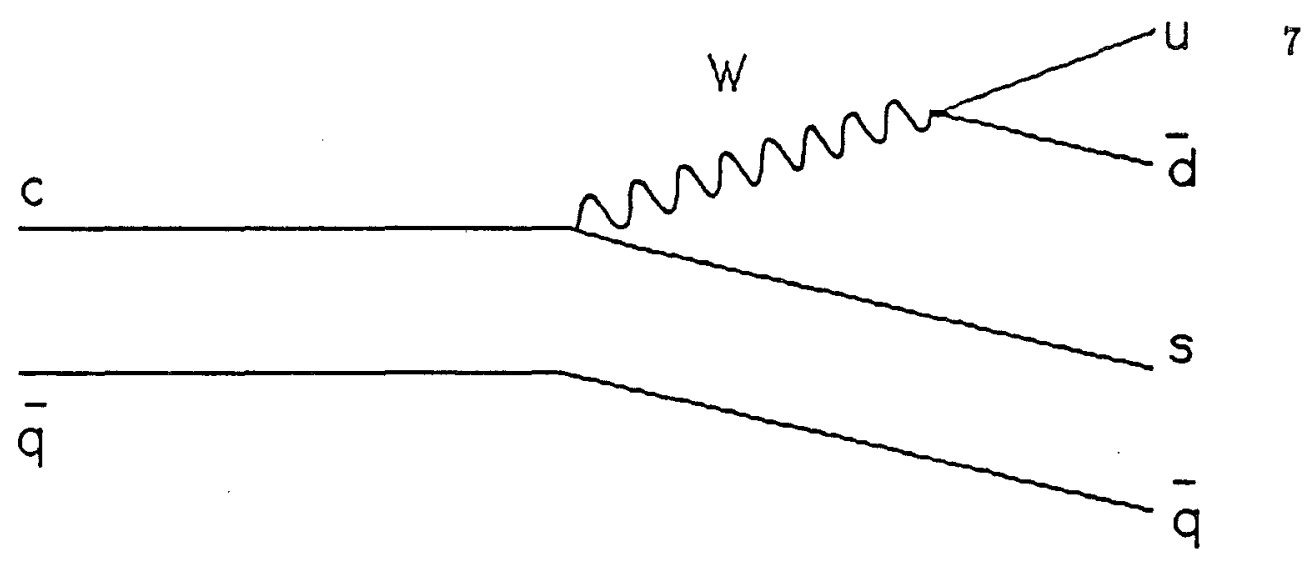

b)
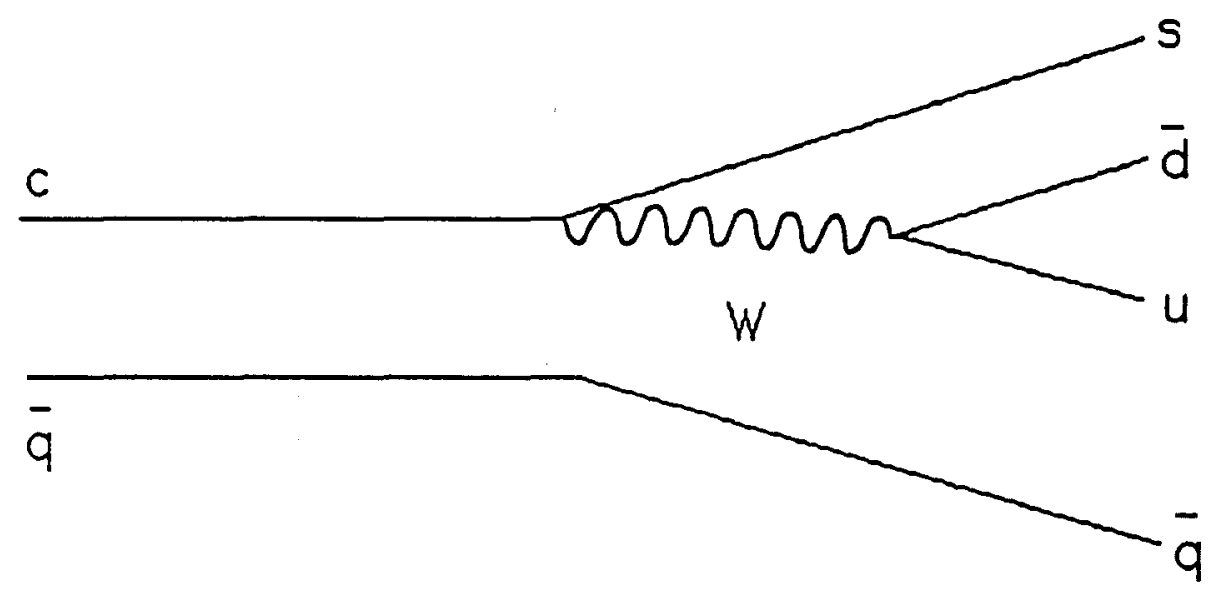

c)

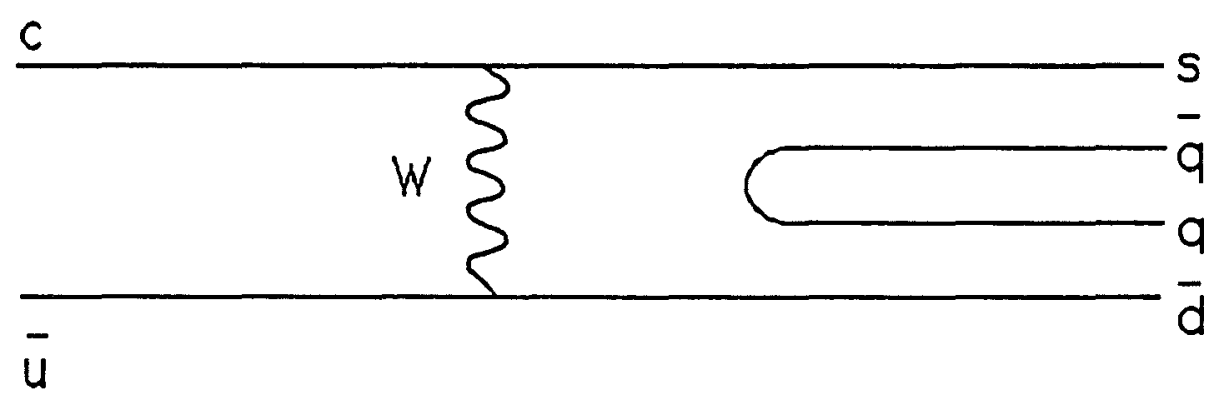

d)

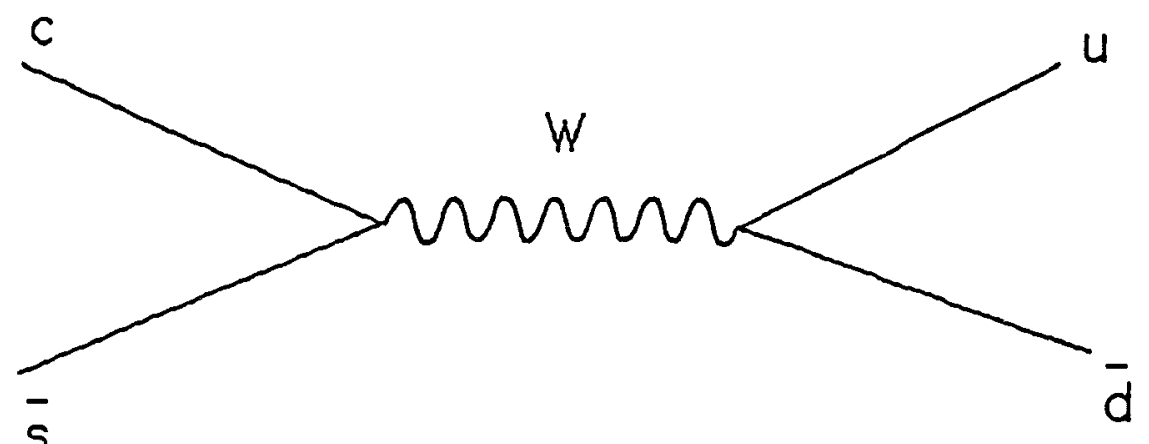

Figure 1.3. Lowest Order Diagrams for Charm Decays: a) external W-emission; b) internal W-emission; c) W-exchange; d) W-annihilation 
quarks are confined within hadrons so that weak decays of hadrons necessarily involve strong interactions. In order to get a qualitative feeling for the dynamics of this decay let's consider the following picture:

1. In general hadrons are complicated superpositions of an infinite number of shortlived quark and gluon configurations. The simplest of these configurations is the valence quark state $(Q \bar{q})$.

2. Due to the large mass of the $\mathrm{W}$ boson, the weak interaction time $\left(t_{w} \sim 1 / M_{w}\right)$ is considerably shorter than the typical lifetime $\left(t \sim 1 / \Lambda_{q c d}\right)$ of these fluctuations. So in one of the configurations, the heavy quark can transform into a light quark via charged current interaction (emission of a W).

3. The newly created quark and the other constituents ("brown muck") move freely within confinement radius, but as the separation increases, the confinement forces cause the stored energy to materialize producing the final state hadrons.

This qualitative picture is illustrated in Fig. 1.2.

We notice that in this picture the free quark decay gets modified at two distance scales "short" and "long". The former comprising the hard gluon interactions among the four quarks (heavy quark plus 3 newly produced) and latter being soft gluon radiation. Bound state wavefunctions and final state interactions are characterized by ordinary hadronic scales $\left(t_{s} \sim 1 / \mathcal{M}_{\text {charm }}\right)$. In order to be able to get quantitative answers one usually makes the assumption that these two types of interactions could be separated in spite of the complexity of their interplay. Thus "long" distance effects get absorbed in the wave functions of the initial and final state hadrons while asymptotic freedom of QCD allows us to treat short distance effects as a perturbation to the free quark decay IIamiltonian given by equation 1.7. The weak decay amplitude 


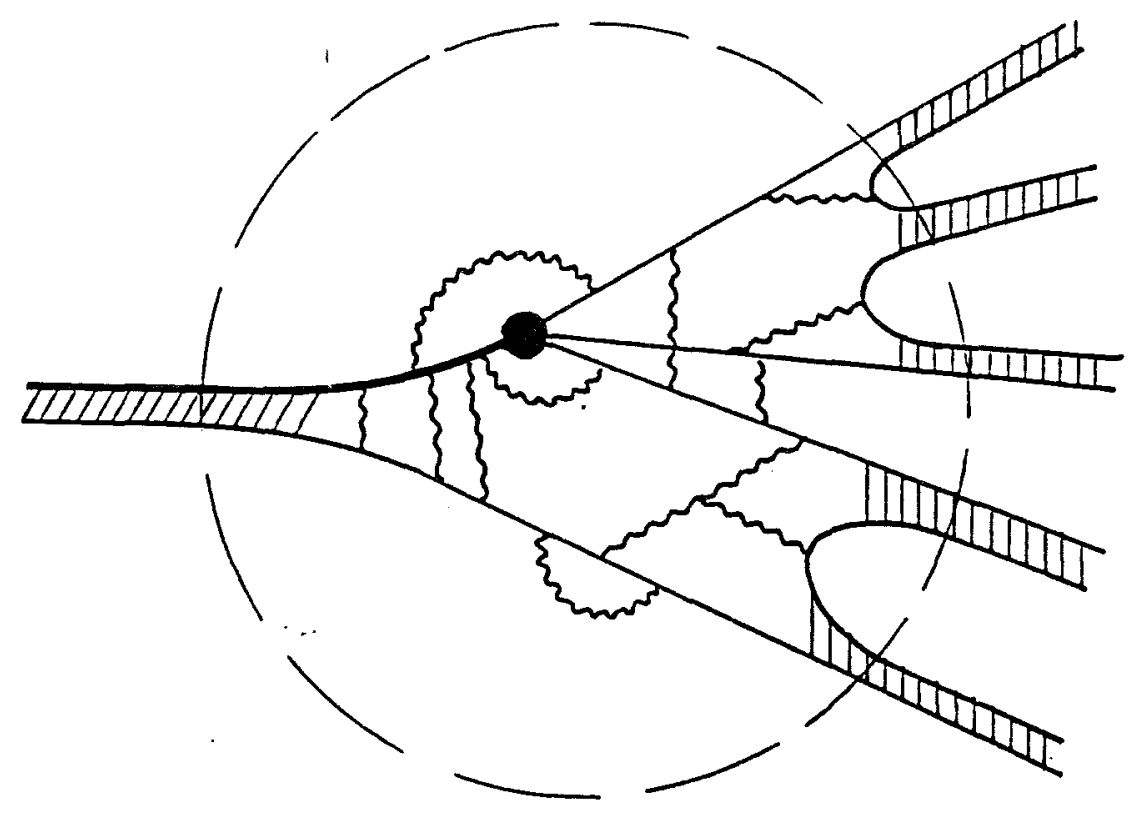

Figure 1.2. Qualitative picture of the weak decay of a heavy meson into five hadrons. Thick line represents the heavy quark, wavy lines the gluons, black dot the weak interaction, and dashed circle the boundary of the confinement region.

is then given by

$$
\mathcal{A}(a \rightarrow b+c+d+e . .)=<b c d . .\left|\mathcal{H}_{e f f}\right| a>
$$

Thus the calculation of the weak decay amplitude is broken into two steps: first the derivation of the effective weak Hamiltonian, and second the calculation of the corresponding matrix element. The second is much more difficult since long distance aspects of QCD cannot yet be calculated from first principles. With this in mind two options are open; phenomenological models or lattice gauge calculations from first principles. 


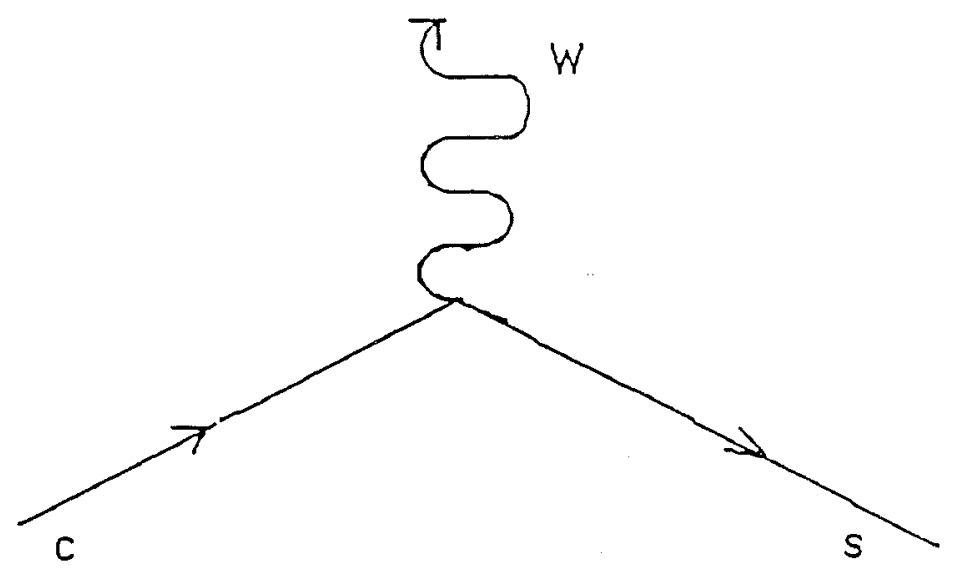

Figure 1.1. Heavy Quark Decay Diagram

where $\mathcal{J}^{\mu}$ represents a sum of quark and lepton currents, $\mathcal{W}_{\mu}$ are the intermediate vector boson fields and $g$ is the coupling strength of vector fields to the current $\mathcal{J}^{\mu}$. Quark currents in terms of mass eigenstates are given by

$$
j^{\mu}=(\bar{u}, \bar{c}, \bar{t}) \gamma^{\mu}\left(1-\gamma^{5}\right) V\left(\begin{array}{l}
d \\
s \\
b
\end{array}\right)
$$

$V$ is the famous $3 \times 3$ Kobayashi-Maskawa(CKM) matrix relating mass eigenstates to the weak eigenstates [Kob 73].

$$
\left(\begin{array}{lll}
V_{u d} & V_{u s} & V_{u b} \\
V_{c d} & V_{c s} & V_{c b} \\
V_{t d} & V_{t s} & V_{t b}
\end{array}\right)
$$

The elements of this matrix act as coupling constants for the relevant pair of quarks. In the case of a model with 3 generations of quarks it is possible to parametrize this matrix in terms of three rotation angles $\theta_{i}(i=1,2,3)$ and one non-trivial phase $\delta$ by 
redefining the phases of the quark fields. One of the conventional parametrizations for $\mathrm{V}$ [Cha 84] is given by

$$
\left(\begin{array}{ccc}
c_{12} c_{13} & s_{12} c_{13} & s_{13} e^{-i \delta_{13}} \\
-s_{12} c_{23}-c_{12} s_{23} s_{13} e^{i \delta_{13}} & c_{12} c_{23}-s_{12} s_{23} s_{13} e^{i \delta_{13}} & s_{23} c_{13} \\
s_{12} s_{23}-c_{12} c_{23} s_{13} e^{i \delta_{13}} & -c_{12} s_{23} s_{13} e^{i \delta_{13}} & c_{23} c_{13}
\end{array}\right)
$$

where $c_{i j}=\cos \theta_{i j}$ and $s_{i j}=\sin \theta_{i j}$, with $i$ and $j$ being "generation" labels, $\{i, j=$ $1,2,3\}$. Note that in the standard model the elements of the matrix $V_{i j}$ are fundamental parameters but are not predicted by the model; hence their values must be determined experimentally.

The weak transition amplitude from an intial to a final state $i \rightarrow f$ (in the second order of the weak coupling) is given by [Ruck 83]

$$
\mathcal{M}_{i f}=<f\left|\mathcal{H}_{W}\right| i>
$$

with

$$
\mathcal{H}_{W}=\frac{g_{W}^{2}}{8 i} \int d^{4} x \mathcal{D}_{\mu \nu}\left(x, m_{W}^{2}\right) T\left[\mathcal{J}_{+}^{\mu}(x) \mathcal{J}_{-}^{\nu}(0)+\text { h.c }\right]
$$

where $\mathcal{D}_{\mu \nu}$ is the $\mathcal{W}$ propagator and $T$ denotes time ordering. For established heavy quarks like charm and beauty, the momentum transfer involved in weak decays is considerably smaller then the $\mathcal{W}$ mass. Thus it is appropriate to take the limit $\mathcal{M}_{W} \rightarrow \infty$ in eq. 1.6 which yields the effective low energy Hamiltonian :

$$
\mathcal{H}_{\text {eff }}=\frac{G_{F}}{\sqrt{ } 2}\left(\mathcal{J}_{+}^{\mu} \mathcal{J}_{-\mu}+h . c\right)
$$

The Hamiltonian written above is appropriate for the free quark decays. In reality, 
processing and analysis techniques employed to extract the the D meson signals. The lifetime measurement technique together with the measurements perfomed are discussed in Chapter 5. The results are summarized, and concluding remarks are given in Chapter 6 . 


\section{CHAPTER 2}

\section{The E-687 Experiment}

The E-687 experiment was commissioned in the summer of 1987 and was in operation periodically through January of 1992 in the Wide Band Photon Laboratory

located at Fermi National Accelerator Laboratory (FNAL) in Batavia, Illinois, USA.

This experiment was designed to study the interactions of high energy photons with matter, in particular the production and decay of states containing heavy quarks (i.e. charm and beauty). The equipment consisted of two parts; Beam Line and the Detector. The former was designed to create and transport the high energy photon beam to the experimental target located at the upstream end of the experimental hall housing the Detector designed to detect the products of photon-nucleon interactions occuring in the target. The detection is accomplished by a large-acceptance, high-rate multi-particle spectrometer located just downstream of the target.

\subsection{Photon Beam}

The beam line was commissioned during the summer of 1985 and upgraded during 1989. The photon beam is a tertiary beam of the bremsstrahlung type derived from $800 \mathrm{GeV}$ protons.

The protons are derived from a hydrogen gas which is ionized and then accelerated to $800 \mathrm{GeV}$, in five stages of the Fermilab accelerator complex. The last stage is the Tevatron, a $1 \mathrm{~km}$ radius ring of superconducting magnets. The Tevatron produced 


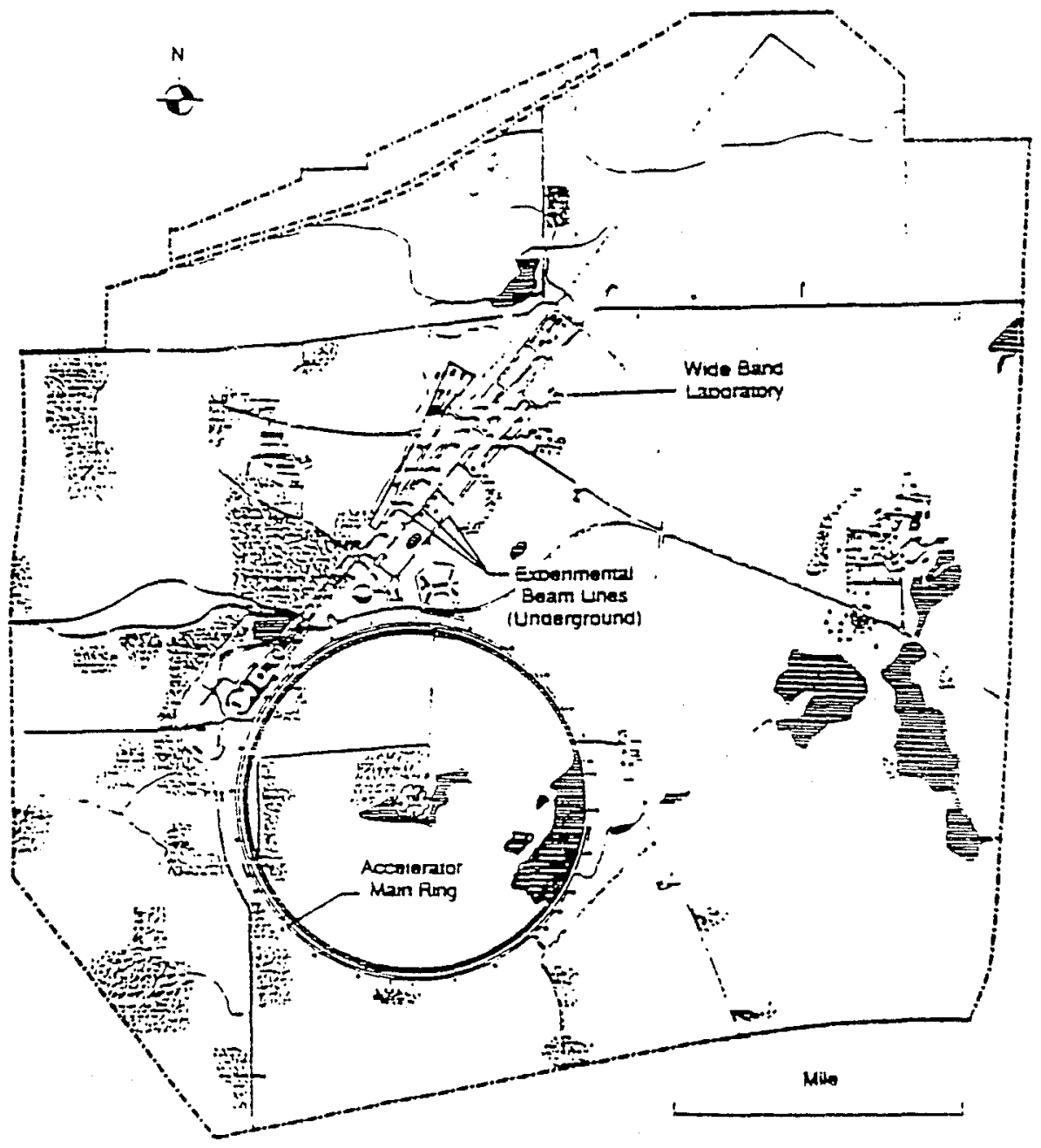

Figure 2.1. Layout of the beamlines at Fermilab

intensity of $\sim 2 \times 10^{13}$ protons over a 20 second spill. Having reached $800 \mathrm{GeV}$ protons are extracted from the Tevatron ring into the Switchyard where the available beam is distributed to the three experimental beamlines Meson, Neutrino and Proton. The Wide Band beam line was a subsidiary of the Proton line. A schematic of the fixed target complex at Fermilab is shown in Fig. 2.1 The photon beam formation and its energy determination (tagging) are described in the following two subsections. 


\subsubsection{Formation of the Photon Beam}

The Wide Band beamline (see Fig. 2.2 usually received $2-5 \times 10^{12}$ protons per spill. These protons were used to produce photons in several steps involving three interactions and a decay process. This rather involved procedure is employed in order to produce a high energy and high purity photon beam free of contamination of neutral hadrons.

Technical description of the beamline is given elsewhere [Fra 93]. Only an outline of the whole procedure will be presented here. Protons delivered to the Wide Band beamline were first made to interact in a beryllium/deuterium $\operatorname{target}^{1}$ (representing one interaction length of material) producing many types of reaction products including charged $\left(\pi^{+}, \pi^{-}, K^{+}, K^{-}, p^{+}\right.$, and $\left.p^{-}\right)$and neutral hadrons $\left(\pi^{0}, \gamma, n, K^{0}\right)$. All charged particles emerging from the above interaction are removed from the beam line by a set of sweeping magnets while the neutral particles are allowed to continue straight down the beam pipe undeflected, passing through an aperture ("collimator") that defines the angular acceptance of the beam. After the collimator the composition of the beam is $\sim 50 \%$ neutrons $\sim 50 \%$ photons (mostly from electromagnetic decays of $\pi^{0}$ 's) and a small but significant component of $K_{L}^{0}$ (around $3 \%$ ). The next step involves the purification of the photon beam. This is done by passing the neutral beam through a lead foil (one radiation length thick) called the "converter" that creates principally electron-positron pairs from incident photons. These $e^{+} e^{-}$pairs are steered away from the beam centerline by a conventional beam transport consisting of dipole and quadrupole magnets around a "neutral dump" while neutral hadrons (neutrons and $K_{L}^{0}$ ) together with unconverted photons get absorbed in the dump. The electrons emerging from the lead converter have a wide range of momenta and angles hence the transport system has to have a large acceptance if high fluxes are

'The beryllium target was replaced by the Deuterium target for the latter's much higher photon yield. 
Table 2.1. Wide Band beam properties

\begin{tabular}{|l|l|}
\hline Quantity & Value \\
\hline Horizontal spot size at production target & $\delta x= \pm 1 \mathrm{~mm}$ \\
Vertical spot size at production target & $\delta y= \pm 1 \mathrm{~mm}$ \\
Geometric horizontal angle accepted & $\delta \theta_{x}= \pm 1 \mathrm{mrad}$ \\
Geometric vertical angle accepted & $\delta \theta_{y}= \pm 0.75 \mathrm{mrad}$ \\
Geometric solid angle accepted & $\Delta \Omega=6.0 \mu$ sterad \\
Maximum momentum bite & $\frac{\Delta P}{P} \approx \pm 15 \%$ \\
Horizontal spot size at expermental target & $\delta x= \pm 1.25 \mathrm{~cm}$ \\
Vertical spot size at expermental target & $\delta y= \pm 0.75 \mathrm{~cm}$ \\
Horizontal divergence at expermental target & $\delta \theta_{x}= \pm 0.6 \mathrm{mrad}$ \\
Vertical divergence at expermental target & $\delta \theta_{y}= \pm 0.5 \mathrm{mrad}$ \\
\hline
\end{tabular}

to be obtained. The mean momentum accepted by this system was $350 \mathrm{GeV}$ with a $\pm 15 \%$ spread making the "Wide Band" name a synonym for the large momentum acceptance. Lastly electrons/positrons of selected momenta were made to radiate photons (bremsstrahlung process) by passing them through a lead foil $20 \%$ of a radiation length in thickness. The recoil electrons were deflected into an electromagnetic calorimeter by a string of dipole sweeping magnets allowing the photons to continue forward onto the experimental target. During standard running conditions this beam line delivered $\sim 2 \times 10^{-5}$ electrons per incident proton. The hadronic contamination in the beam is produced by a sequence of processes each of which has relatively low probability. The resultant background from neutral hadrons has been measured to be $10^{-5}$ neutrons per photon. Hence less than $1 \%$ of hadronic interactions in the experimental target are produced by neutral hadrons. The properties of the Wide Band beam are summarized in Table 2.1. After the initial run in 1987-88 the E-687 Collaboration upgraded the beamline [Kas 88] by instrumenting the positron side of the charged particle transport system with the goal of doubleing the photon yield. The positron arm was used only for test purposes due to its much higher background levels. 


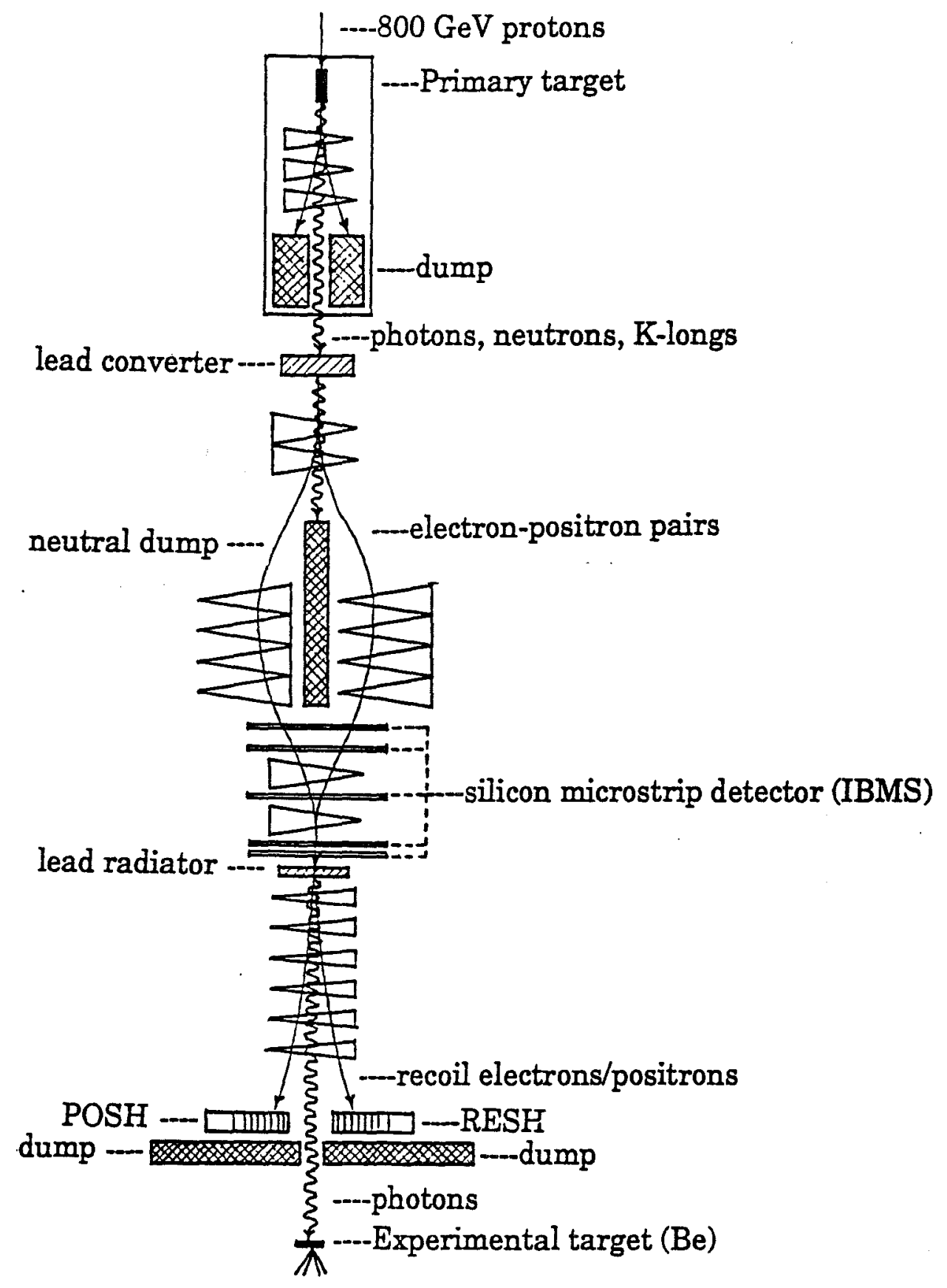

Figure 2.2. The E-687 beam line layout 


\subsubsection{Energy determination of the Photon Beam}

The energy of the interacting photon is determined on an event-by-event basis by measuring the electron momentum before and after the radiator, as well as by measuring the energy of any non-interacting photons created at the same time through multiple bremsstrahlung. This is accomplished by three independent detectors : Incident Beam Magnetic Spectrometer (IBMS), Radiated Electron Shower Hodoscope (RESH), and Beam Gamma Monitor (BGM). Hence the energy of an interacting photon is given by

$$
E_{\gamma}^{\text {int }}=E_{\mathrm{IBMS}}-E_{\mathrm{RESH}}-E_{\mathrm{BGM}}
$$

The uncertainty in each of these terms limits the resolution in the estimate of the photon energy. Originall ${ }^{2} \mathrm{y}$ the largest contributor to the resolution was the $\pm 50 \mathrm{GeV}$ energy spread of the incident electron beam. To improve the photon energy resolution the IBMS detector was constructed during the 1989 shutdown. This spectrometer consisted of 5 large area silicon microstrip planes which measure the bend angle of the electron/positrons as they pass through the transport system towards the radiator. Silicon microstrip planes are $300 \mu \mathrm{m}$ thick with a pitch of $300 \mu \mathrm{m}$, providing a $2.2 \%$ electron momentum resolution, which is somewhat better than the energy resolution of the RESH. Silicon detectors were chosen for their ability to sustain a high intensity beam (in excess of $10^{7}$ particles per second), intrinsically fast time response, and robustness compared to drift chambers. The total system had 1280 readout channels (256 channels/plane).

All charged particles emerging from the radiator are swept away from the beam

\footnotetext{
${ }^{2}$ During the initial run of E-687 in $1987 / 88$.
} 


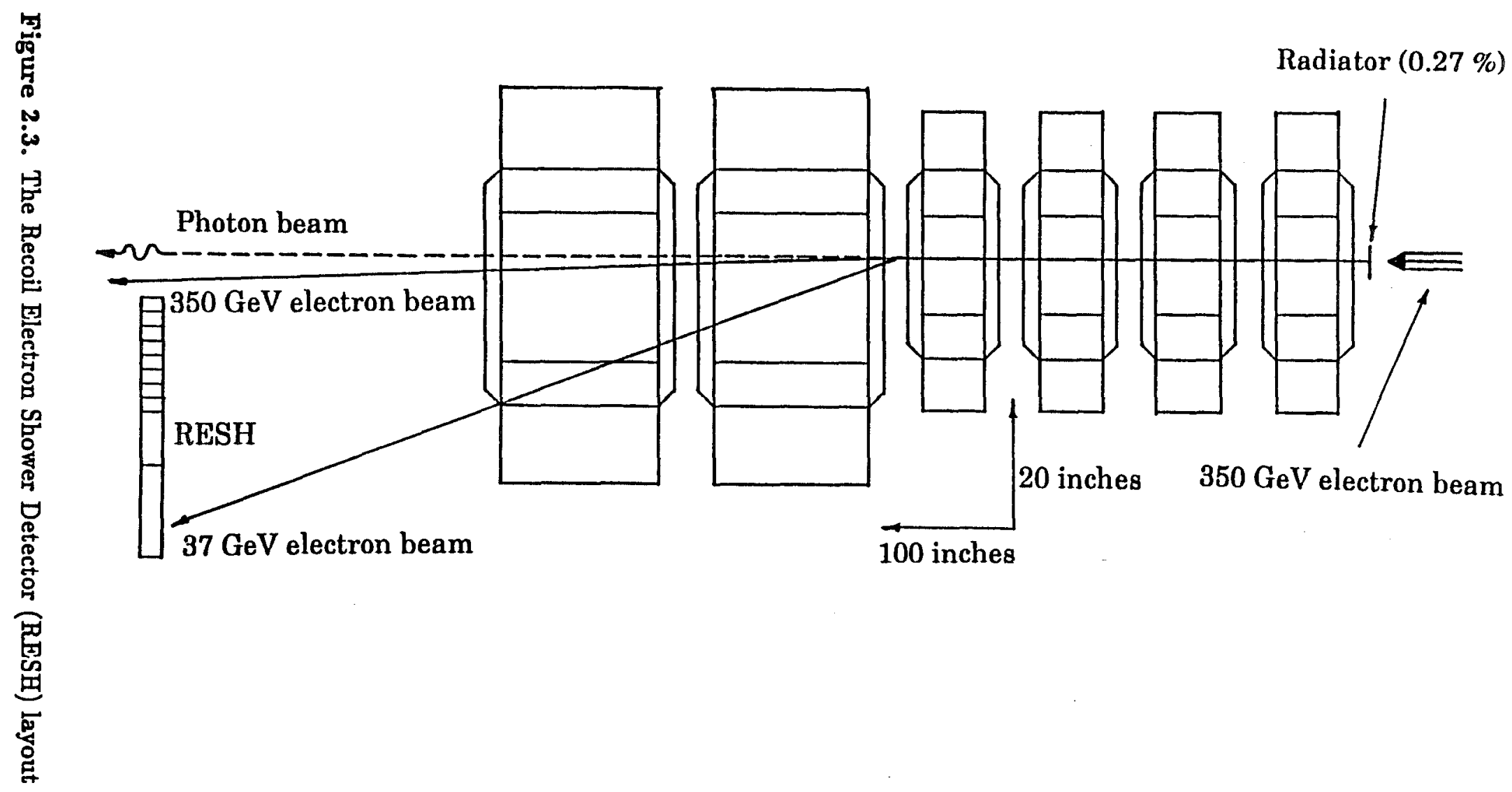




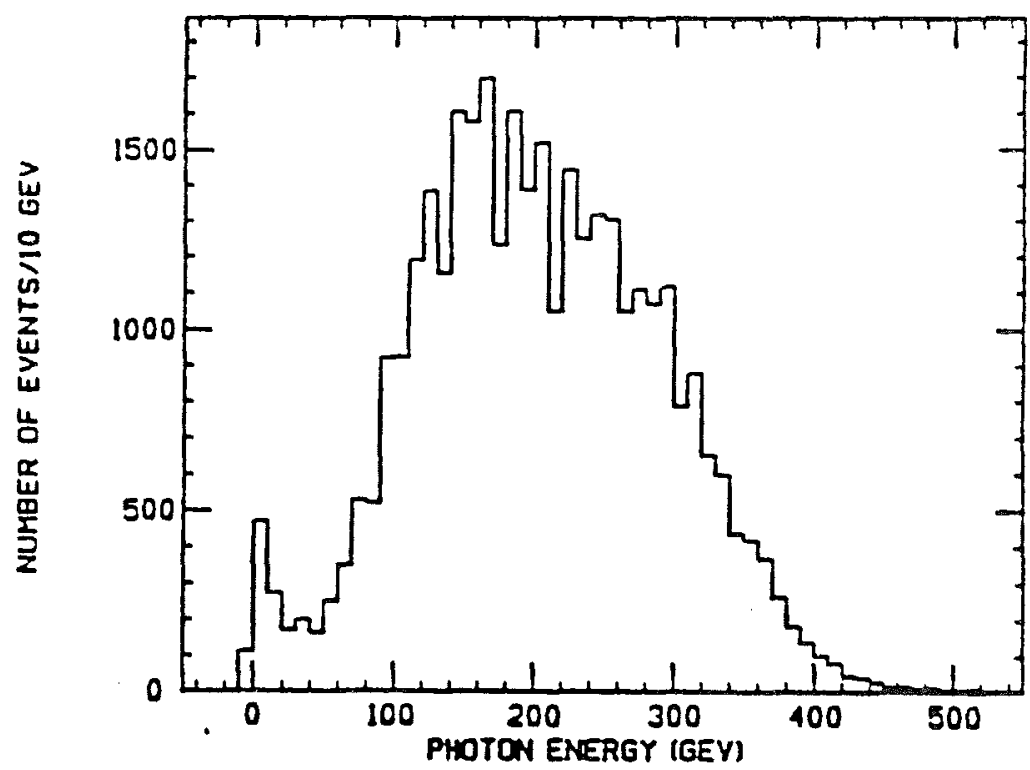

Figure 2.4. Tagged Photon Spectrum

centerline by a set of sweeping magnet ${ }^{3}$ s into a beam dump. Electrons which have not, interacted in the radiator will be deflected less than those which did interact, due to their higher momenta. In order to detect only electrons that have lost a substantial amount of their energy to bremsstrahlung, the RESH detector was placed $10.4 \mathrm{~cm}$ transverse to the beam pipe downstream of the sweeping magnets in front of the electron dump. The transverse offset of this detector established a minimum tagged electron energy loss to be roughly $110 \mathrm{GeV}$.

The RESH detector itself (see Fig. 2.3 consisted of 11 cells oriented transversely to the beam direction. Each cell was a scintillator shower counter made up of lead-lucite layers 24 radiation lengths deep outfitted with a single RCA 8575 photomultiplier tube. The energy of each recoil electron was determined from the PMT pulse height as well as by the momentum determination from the kick of the sweeping magnets and the transverse position of the hit cell.

$3 \int B \times d l=89.5$ KGauss-meter deflecting $350 \mathrm{GeV}$ electrons $10.24 \mathrm{~cm}$ from the photon beamline. 
The RESH detector was included in the second level trigger in order to effectively harden the $1 / k$ photon energy spectrum by getting rid of low energy photons.

Electrons that interact in the radiator often produce more than one photon but typically only one interacts in the experimental target. The energy of non-interacting photons was measured in a zero-degree shower counter (BGM) located at the downstream end of the spectrometer. The BGM detector is a lead-lucite shower counter 24 radiation lengths deep read out by a single Amperex 58AVP photomultiplier tube.

\subsection{Spectrometer}

The purpose of E-687 detector [Fra 92] is to detect the products of high energy photonnucleon interactions occurring in the experimental target. The layout of the experiment is shown in Fig. 2.5. To achieve good geometrical acceptance it was divided into two detector regions defined by the apertures of two analysis magnets: inner with $\mathrm{x}-\mathrm{y}$ acceptance $\theta_{\mathrm{x}}^{\max } \pm 30 \mathrm{mrad}$ and $\theta_{\mathrm{y}}^{\max } \pm 50 \mathrm{mrad}$, and outer extending the acceptance to $\theta_{x}^{\max } \pm 100 \mathrm{mrad}$ and $\theta_{\mathbf{y}}^{\max } \pm 150 \mathrm{mrad}$.

The spectrometer was capable of charged particle tracking and identification, as well as detection of electromagnetic and hadronic showers. The tracking system consisted of a high resolution silicon microstrip system just downstream of the target and a set of multi-wire proportional chambers with two analysis magnets following it. Charged particle identification was performed by a set of multicell Čerenkov counters, two muon detectors and two electromagnetic calorimeters. The latter two devices were also used for photon and $\pi^{0}$ detection. The hadron calorimeter was primarily used for triggering purposes as well as neutral hadron detection. The exact location and size of each detector is given in Tables 2.2 and 2.3.

A subsystem of detectors (TRIGGER) was designed to select Good events. These detectors enabled the experiment to ignore photon electromagnetic interactions which comprise $99 \%$ of all photon interactions in the target as well as spurious interactions 


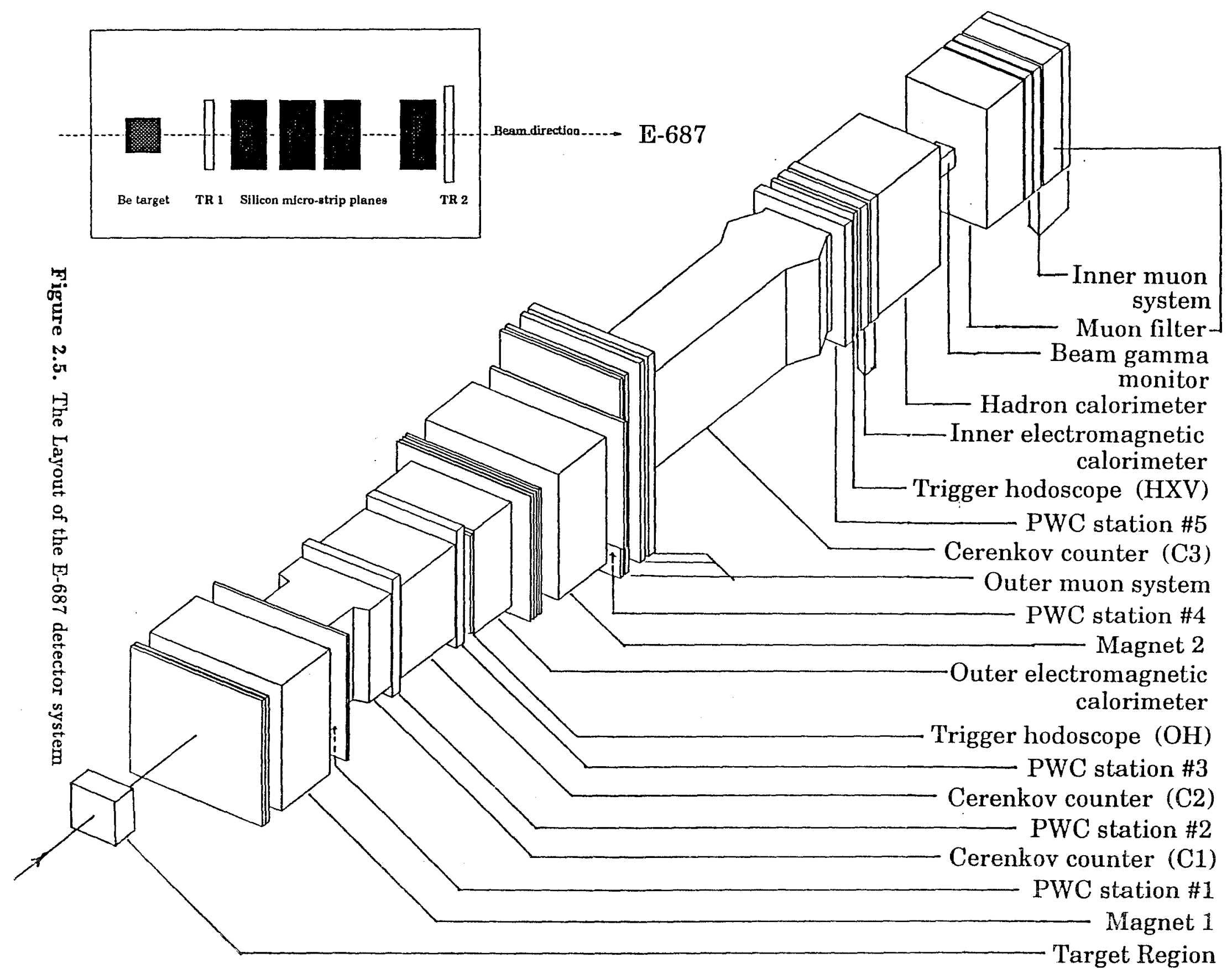


Table 2.2. Table with individual subsystem dimensions and their location

\begin{tabular}{|l|c|c|c|}
\hline Device & Z location $^{a}(\mathrm{~cm})$ & Z length $(\mathrm{cm})$ & Area $\left(\mathrm{cm}^{2}\right)$ \\
\hline Be Target & -3.11 & 4.8 & $2.54 \times 2.54$ \\
SSD-1 & 4.63 & 1.07 & $2.48 \times 3.50$ \\
SSD-2 & 10.69 & 1.07 & $4.96 \times 4.96$ \\
SSD-3 & 16.72 & 1.07 & $4.96 \times 4.96$ \\
SSD-4 & 28.66 & 1.07 & $4.96 \times 4.96$ \\
M1 Up. Shield hole & 77.44 & 17.78 & $25.4 \times 101.6$ \\
M1 Aperture & 220.95 & 167.64 & $76.2 \times 127.0$ \\
M1 Dwn. Shield hole & 370.17 & 8.90 & $76.2 \times 127.0$ \\
P0 & 405.08 & 17.78 & $76.2 \times 127.0$ \\
C1 & 519.75 & 187.96 & $132.1 \times 182.9$ \\
P1 & 644.26 & 17.78 & $152.4 \times 228.6$ \\
C2 & 757.00 & 187.96 & $152.4 \times 228.6$ \\
P2 & 878.47 & 17.78 & $152.4 \times 228.6$ \\
OH & 896.00 & 2.00 & $270.0 \times 250.0$ \\
OH Aperture & 896.00 & 2.00 & $48.6 \times 83.2$ \\
OE & 962.99 & 132.4 & $270.0 \times 250.0$ \\
OE Aperture & 962.99 & 132.4 & $48.6 \times 83.2$ \\
M2 Up. Shield hole & 1091.43 & 8.90 & $76.2 \times 127.0$ \\
M2 Aperture & 1238.11 & 167.64 & $76.2 \times 127.0$ \\
M2 Dwn. Shield hole & 1383.52 & 8.90 & $76.2 \times 127.0$ \\
Outer $\mu$ Prop. X & 1399.24 & 10.0 & $304.8 \times 508.0$ \\
Outer $\mu$ Prop. X hole & 1399.24 & 10.0 & $101.6 \times 162.6$ \\
Outer $\mu$ Prop. Y & 1416.94 & 10.0 & $304.8 \times 508.0$ \\
Outer $\mu$ Prop. Y hole & 1416.94 & 10.0 & $101.6 \times 162.6$ \\
P3 & 1442.6 & 17.78 & $76.2 \times 127.0$ \\
\hline
\end{tabular}

${ }^{a} \mathrm{Z}$ position measured at the center of the device with respect to the Granite Block 
Table 2.3. Table with individual subsystem dimensions and their location

\begin{tabular}{|l|c|c|c|}
\hline Device & Z location $(\mathrm{cm})$ & Z length $(\mathrm{cm})$ & Area $\left(\mathrm{cm}^{2}\right)$ \\
\hline Outer $\mu$ Scint. Y & 1474.56 & 15.0 & $304.8 \times 487.7$ \\
Outer $\mu$ Scint. Y hole & 1474.56 & 15.0 & $121.9 \times 152.4$ \\
Outer $\mu$ Scint. X & 1505.06 & 22.8 & $304.8 \times 487.7$ \\
Outer $\mu$ Scint. X hole & 1505.06 & 22.8 & $121.9 \times 152.4$ \\
C3 & 1884.42 & 703.58 & $190.5 \times 228.6$ \\
P4 & 2288.89 & 17.78 & $101.6 \times 152.4$ \\
H $\times \mathrm{V}$ & 2328.19 & 9.22 & $141.6 \times 246.0$ \\
H $\times$ V gap & 2328.19 & 9.22 & $7.1 \times 365.8$ \\
IE & 2399.7 & 76.84 & $123.1 \times 123.1$ \\
IE hole & 2399.7 & 76.84 & $10.2 \times 10.2$ \\
Hadron Calorimeter & 2569.8 & 219.3 & $203.2 \times 304.8$ \\
Hadron Calorimeter hole & 2569.8 & 219.3 & $30.0($ dia. $)$ \\
Beam Gamma Monitor & 2704.0 & 40.6 & $25.4 \times 22.9$ \\
Central Hadr. Calorimeter & 2778.0 & 101.6 & $45.7 \times 45.7$ \\
Muon filter 1 & 2895.7 & 128.6 & $231.1 \times 330.2$ \\
Inner $\mu$ Prop. X & 2973.5 & 10.0 & $203.2 \times 304.8$ \\
Inner $\mu$ Prop. Y & 2993.2 & 10.0 & $203.2 \times 304.8$ \\
Inner $\mu$ Scint. X & 3012.5 & 15.3 & $213.4 \times 304.8$ \\
Inner $\mu$ Scint. Y & 3036.1 & 9.0 & $203.2 \times 304.8$ \\
Muon filter 2 & 3079.7 & 63.0 & $231.1 \times 330.2$ \\
Inner $\mu$ Prop. X & 3139.0 & 10.0 & $203.2 \times 304.8$ \\
Inner $\mu$ Prop. Y & 3158.1 & 10.0 & $203.2 \times 304.8$ \\
Inner $\mu$ Scint. Y & 3178.3 & 9.0 & $203.2 \times 304.8$ \\
\hline
\end{tabular}


occurring in the detector. It associated a definite time stamp to each interesting photon interaction.

\subsubsection{Experimental Target}

Over the course of several runs a number of different experimental targets were used and tested. Most of the running was done with beryllium targets of different shapes and sizes. Besides beryllium the experiment used/tested two active targets: a silicon active target and a scintillating fiber target. The silicon active target [Bel 92] consisted of active silicon layers interleaved with beryllium. The scintillating fiber target [Mou 92] was a glass target formed from over $10^{6} 25 \mu \mathrm{m}$ scintillating fibers whose interaction images were recorded by a sophisticated imaging system.

The beryllium target was made up of beryllium segments typically $2.54 \mathrm{~cm}$ across stacked to form a 4-5 cm long target having $10 \%$ of an interaction length and about $11 \%$ of a radiation length. Beryllium targets of larger area were periodically used for beam profile monitoring. The beryllium target was a primary choice due to its favorable ratio of interaction to radiation length, as well as for its well known behavior and simple handling procedure.

\subsubsection{Charged Particle Tracking}

The silicon microstrip detector system (SSD) was located immediately after the experimental target to provide charged particle tracking between the target and the first analysis magnet. Silicon technology was chosen in order to achieve very high position accuracy needed in this region to separate the production and decay vertices of particles containing heavy flavors as well as its ability to sustain high rates. It consisted of 12 microstrip planes grouped in 4 stations with 3 detectors each measuring $i, j$ and $k$ coordinates respectively: $i$ at $-135^{\circ}, j$ at $-45^{\circ}$ and $k$ at $-90^{\circ}$ with respect 


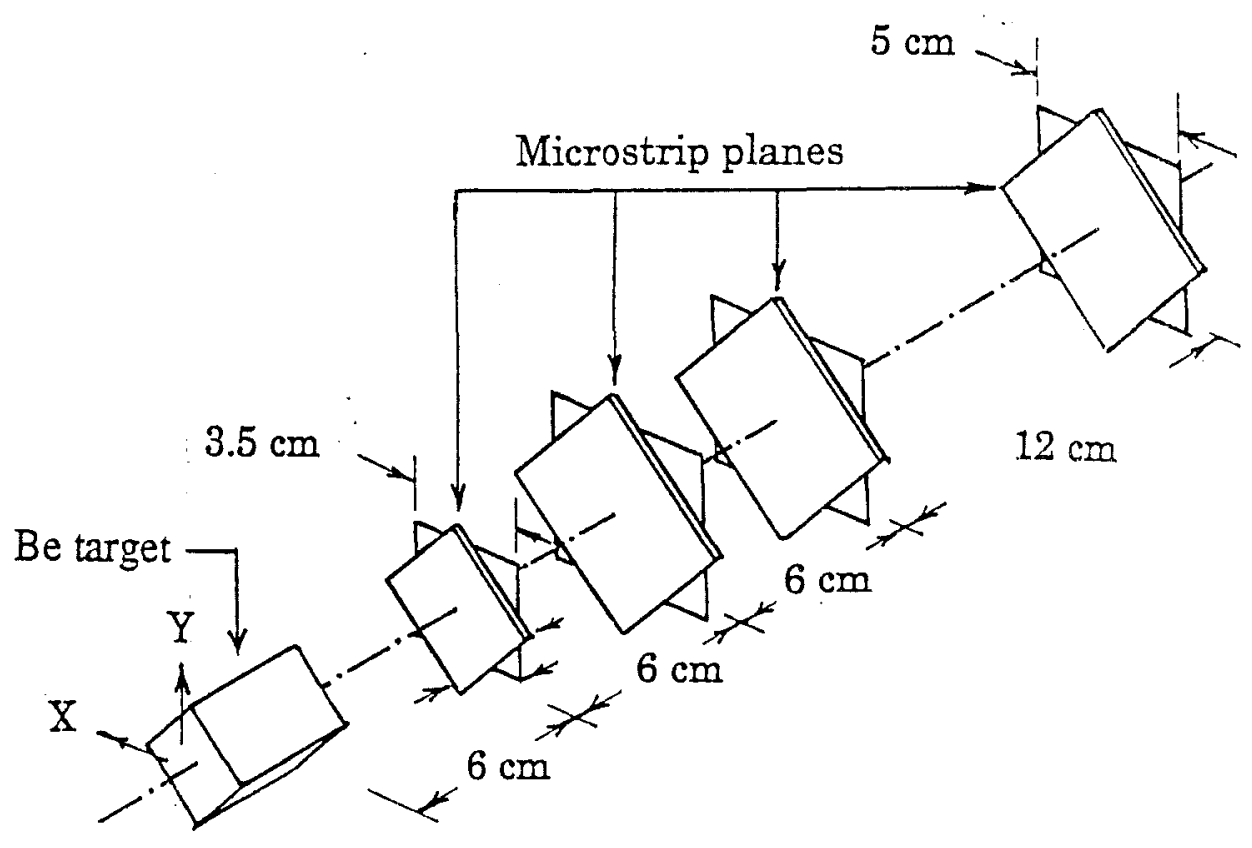

Figure 2.6. The layout of the silicon microstrip detector

to the horizontal. The schematic of the SSD system is shown in Fig. 2.6. Each plane consisted of 688 silicon strips with nominal pitch of $50 \mu \mathrm{m}$ (25 $\mu \mathrm{m}$ in the first station). The resolution of inner region in each plane was two times the resolution of the outer region. The geometry of the SSD system is described in Table 2.4.

The pulse height from each strip is read out via front-end preamplifier, remote end amplifier, and a custom-built charge integrating FLASH ADC [Bel 86] [Inz 86]. The amount of charge released per strip allowed a simple estimate of the number of tracks involved. The overall detection efficiency of every plane was measured to be better than $99 \%$. Position resolution expressed as extrapolated transverse error to the mean interaction point in the experimental target $(7 \mathrm{~cm}$ upstream of the first microstrip plane) was measured to be $\sim 9 \mu \mathrm{m}$ for infinite momentum tracks crossing the high resolution regions of the twelve planes.

The tracking downstream of the first magnet was performed by a set of conventional multi-wire proportional chambers. This subsystem consisted of 20 signal planes 
Table 2.4. Properties of the silicon microstrip detector

\begin{tabular}{|l|c|c|c|c|}
\hline Z-position & I Station & II Station & II Station & IV Station \\
\hline $1^{\text {st }}$ detector & $-0.5 \mathrm{~cm}$ & $5.5 \mathrm{~cm}$ & $11.5 \mathrm{~cm}$ & 23.5 \\
$2^{\text {nd }}$ detector & $0.0 \mathrm{~cm}$ & $6.0 \mathrm{~cm}$ & $12.0 \mathrm{~cm}$ & 24.0 \\
$3^{\text {rd detector }}$ & $0.5 \mathrm{~cm}$ & $6.5 \mathrm{~cm}$ & $12.5 \mathrm{~cm}$ & 24.5 \\
active area & $2.5 \times 3.5 \mathrm{~cm}^{2}$ & $5 \times 5 \mathrm{~cm}^{2}$ & $5 \times 5 \mathrm{~cm}^{2}$ & $5 \times 5 \mathrm{~cm}^{2}$ \\
High res.area & $1 \times 3.5 \mathrm{~cm}^{2}$ & $2 \times 5 \mathrm{~cm}^{2}$ & $2 \times 5 \mathrm{~cm}^{2}$ & $2 \times 5 \mathrm{~cm}^{2}$ \\
Strip Pitch & $25.5 \mu \mathrm{m}$ & $50.1 \mu \mathrm{m}$ & $50.1 \mu \mathrm{m}$ & $50.1 \mu \mathrm{m}$ \\
No. of channels & $688 \times 3$ & $688 \times 3$ & $688 \times 3$ & $688 \times 3$ \\
\hline
\end{tabular}

${ }^{a}$ Position measured relative to supporting granite block.

grouped into 5 stations. Each station had 4 views $X, Y, U, V$ each with a different wire orientation. The $\mathrm{X}$-view wires run horizontally (non-bend view), Y-view wires vertically (bend-view), while $U$ and $V$ view wires were oriented at $\pm 11.3^{\circ}$ with respect to $Y$ wires. Anode wire spacing was $2-3 \mathrm{~mm}$ depending on the particular station. Each station had either 2296 or 2944 instrumented wires so the total PWC system had over 13000 channels. The ionization medium used was $65 \%$ Argon $/ 35 \%$ Ethane gas mixture which flowed continuously through the system at atmospheric pressure. These planes run typically at voltages to ground between 2.8 and $3.5 \mathrm{kV}$. Signals from the sense wires were amplified and discriminated by pre-amplifiers mounted directly on the stations. These logic signals were then sent to the counting room to be read by the LeCroy 4290 TDC system.

Charged particle momentum was measured by two identical large aperture dipole magnets located 225 and $1240 \mathrm{~cm}$ respectively downstream of the experimental target. The magnets deflected particles in the vertical plane in opposite directions. The transverse momentum kick magnitude was chosen to be $0.4 \mathrm{GeV} / \mathrm{p}$ and $-0.85 \mathrm{GeV} / \mathrm{p}$ respectively so that charged tracks would be refocused to the beam line at the front face of the inner electromagnetic calorimeter. Besides momentum measurement the 
Table 2.5. Analysis magnet specifications

\begin{tabular}{|l|l|}
\hline Quantity & Value \\
\hline Central field & $14.0 \mathrm{KGauss}$ \\
Maximum $P_{t}$ kick & $0.97 \mathrm{GeV} / \mathrm{c}$ \\
Field Uniformity & $\pm 2.5 \%$ \\
Steel yoke length & $167.64 \mathrm{~cm}$ \\
Effective length & $256.54 \mathrm{~cm}$ \\
Effective length/pole length & 1.55 \\
Total length & $264.26 \mathrm{~cm}$ \\
Maximum current & $2500 \mathrm{Amps}$ \\
Voltage at max. current & $160 \mathrm{Volts}$ \\
Total power at max. current & $400 \mathrm{~kW}$ \\
Total flow (50\% glycol) & $118 \mathrm{gpm}$ \\
Pressure drop accross coils & $150 \mathrm{psi}$ \\
Conductor diameter & $4.71 \mathrm{~cm} \times 3.49 \mathrm{~cm}$ \\
Water passage diameter & $1.55 \mathrm{~cm}$ \\
Total weight & $272 \mathrm{Tons}$ \\
\hline
\end{tabular}

magnets dispersed particles easing the effects of clustering in coarse granularity detectors. The steel yoke of the second magnet was used as an absorber for the outer muon detector located at the back of it. Table 2.5 lists the characteristics of these magnets.

\subsection{3 Č Cerenkov system}

The čerenkov system consists of three multicell counters labeled $\mathrm{C} 1, \mathrm{C} 2$ and $\mathrm{C} 3$. These are gas counters operated at atmospheric pressure in the threshold mode. These counters are used to distinguish more massive hadronic secondaries $\left(K^{ \pm}, p, \vec{p}\right)$ from pions $\left(\pi^{ \pm}\right)$in charm decays in order to suppress the combinatorial backgrounds which are endemic to the reconstruction process. In order to achieve this goal, the gases were chosen so that there is a wide range of momentum values over which $\pi^{ \pm}, K^{ \pm}$, and $p^{ \pm}$ may be identified. Table 2.6 lists the main characteristics of the three counters. From 
Table 2.6. Table with Čerenkov system properties

\begin{tabular}{|c|c|c|c|c|c|}
\hline Counter & \multicolumn{3}{|c|}{ Threshold (GeV/c) } & \multirow{2}{*}{ No. of Cells } & Gas \\
\cline { 2 - 4 } & Pion & Kaon & Proton & & \\
\hline C2 & 4.5 & 15.9 & 30.3 & 110 & $\mathrm{HeN}_{2}$ \\
C1 & 8.4 & 29.7 & 56.5 & 90 & $\mathrm{~N}_{2} \mathrm{O}$ \\
C3 & 17.4 & 61.5 & 117 & 100 & $\mathrm{He}$ \\
\hline
\end{tabular}

this table, it is observed that the pions are separable from protons or kaons from 4.5 to $61 \mathrm{GeV} / \mathrm{c}$ while kaons can be separated from protons between $16 \mathrm{GeV} / \mathrm{c}$ and 116 $\mathrm{GeV} / \mathrm{c}$.

The three counters are divided into a high and a low resolution section in order to match the expected density of charged tracks and so minimize the problem of track "confusion" associated with overlapping light distributions. Counters are instrumented with 2, 3 and 5 inch PMTs at which Cerenkov light is projected by either a planar or a focusing mirror. The plan view of three counters is shown in Fig. 2.7.

The Čerenkov counter $\mathrm{C} 1$ is the most upstream counter of the three located just beyond the first analysis magnet between the first and second sets of PWCs. It had 90 instrumented cells and a pion threshold of $8.5 \mathrm{GeV} / \mathrm{c}$ during $1990-91$ run. The active medium was helium-nitrogen gas mixture. The active transverse area of the counter measured $50 \times 80$ inches, while the total length of the gas volume along the beam was 71 inches. Both planar and focusing mirrors were used for light reflection. Typical detected signals for each cell varied from 2.5 to 3.6 photoelectrons.

The second Cerenkov counter $\mathrm{C} 2$ was located behind $\mathrm{C} 1$, and positioned between the second and third stations of PWCs. It had 110 instrumented cells and the lowest overall pion threshold of $4.5 \mathrm{GeV} / \mathrm{c}$. It had an aperture of $90.5 \times 100$ inches and a length of 74 inches in the beam direction. Cerenkov light was reflected by two planar mirrors placed at $\pm 45^{\circ}$ to the beam direction. The inner high resolution region was instrumented by 54 two-inch Thorn EMI 9939A photomultiplier tubes while the outer 


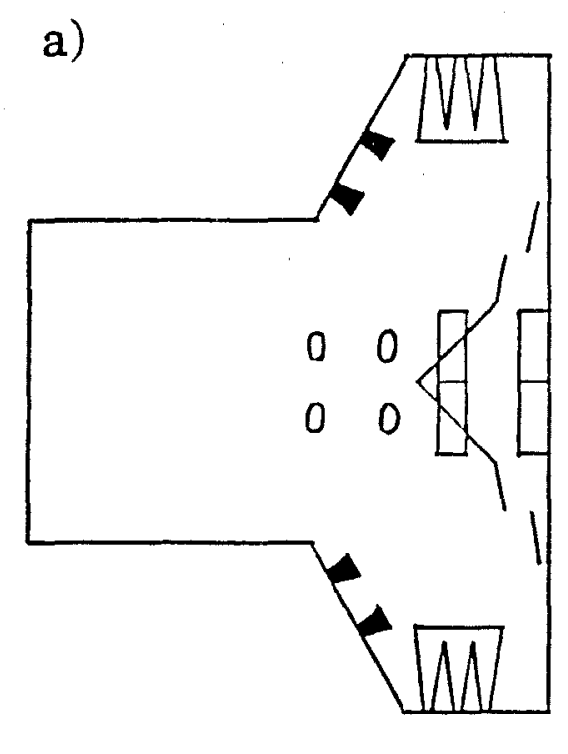

b)
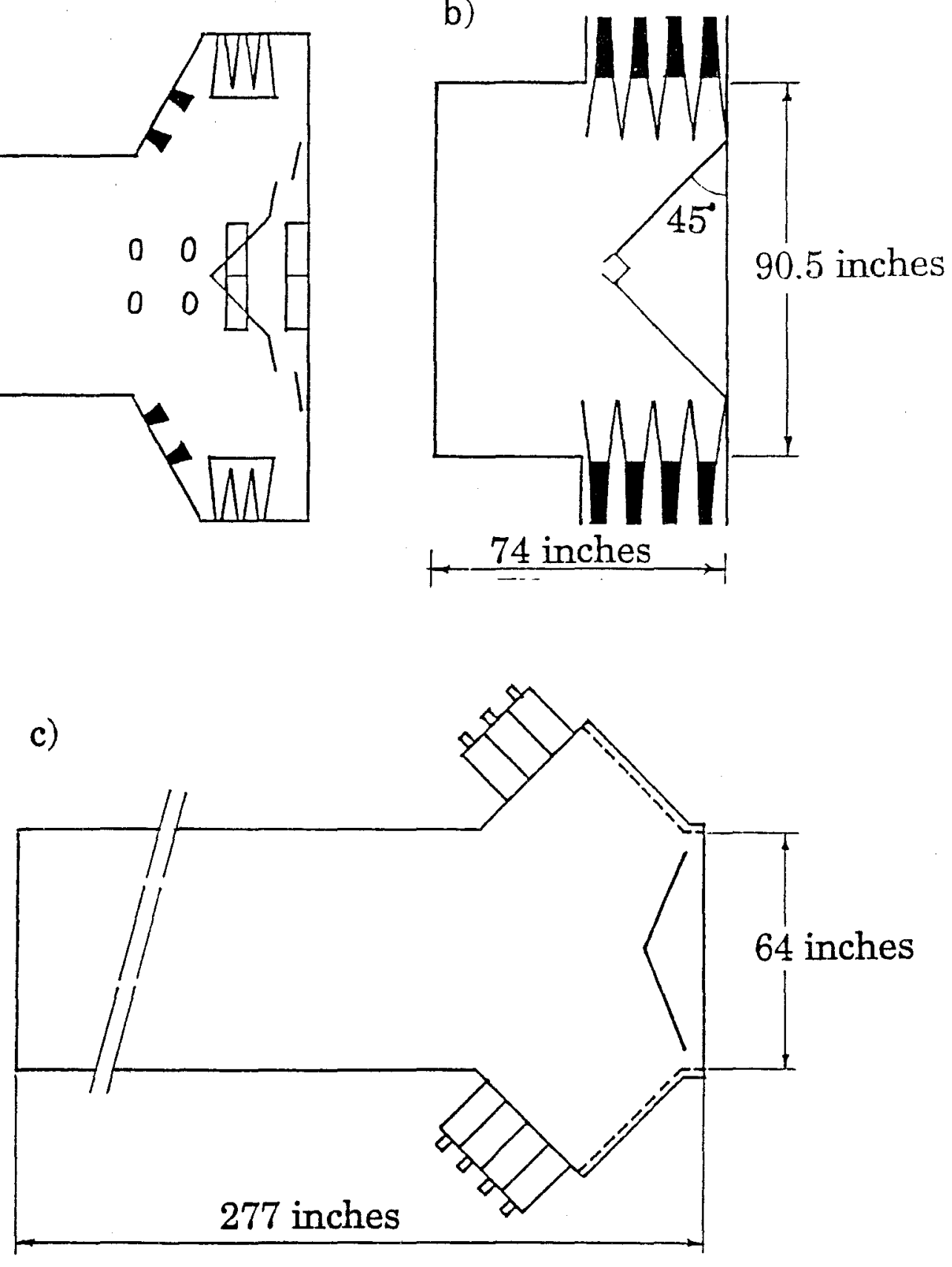

Figure 2.7. Plan view of the three Ceerenkov counters: a) C1; b) C2; c) C3. 
low resolution region was instrumented by 56 five-inch RCA 8854 PMTs. All PMT faces were coated with p-terphenyl waveshifter to improve light detection in the near ultraviolet. The active medium was nitrous oxide gas. The detected signal levels for this counter varied between 5 and 15 photoelectrons per cell.

C3, the last of the three counters was, located downstream of the second magnet, and positioned between the third and fourth PWC stations. It was instrumented with 100 cells and had the highest overall pion threshold of $17 \mathrm{GeV} / \mathrm{c}$. It had aperture of $60 \times 93.25$ inches and a length of 277 inches along the beam line. The counter had 100 spherical glass focusing mirrors with each mirror matching a particular cell. The central and outer sections were instrumented by two-inch and five-inch photomultiplier tubes respectively. Individual response from cells varied from 3 to 17 photoelectrons.

The Čerenkov system had a total of 300 channels instrumented by photomultiplier tubes and custom designed "bases". Each individual pulse was read into a Lecroy Fastbus ADC to be digitized and written to tape. The calibration of this system involved precise determination of cell boundaries as well as the average response in photoelectron equivalents for each cell. The calibration task was performed with muon and electron beams. As a consistency check, the geometry as determined from muon and electron calibrations was compared to the geometry obtained from a photosurvey of these detectors showing very good agreement.

\subsubsection{Electromagnetic Calorimetry}

The electromagnetic calorimetry provides electron identification and photon detection as well as energy determination. Two separate calorimeters covered the inner and outer regions of the detector. The inner electromagnetic calorimeter (IE) was located downstream of the last PWC station while the outer electromagnetic calorimeter (OE) was located in front of the second analysis magnet. Both are lead-scintillator calorimeters, 25 and 21.6 radiation lengths in depth respectively. These two calorime- 
ters are comprised of one-inch and two-inch wide counters/strips ${ }^{4}$ transverse to the beam. Individual pulse height information from a total of 1650 photomultiplier tubes was read out into the Lecroy Fastbus ADCs, where it was digitized and written out to tape.

\subsubsection{Muon system}

The muon system was also divided into inner and outer region detectors. These two subsystems were assembled from scintillator hodoscopes and proportional tubes. The outer muon (OM) detector was mounted right at the back of the second analysis magnet. This location allowed the use of the magnet yoke together with the $\mathrm{OE}$ detector material in front of it as an absorber. The inner muon (IM) detector is the most downstream component of the spectrometer elements located at the very end of the apparatus behind IE, HC and several layers of steel absorber.

\subsubsection{Hadronic Calorimetry}

The hadron calorimetry, designed to detect and measure hadronic energy covered only the inner region of the detector and was located just downstream of the inner electromagnetic calorimeter. It consisted of two separate devices: the main hadron calorimeter $(\mathrm{HC})$ covering the region from $5 \mathrm{mrad}$ to $30 \mathrm{mrad}$ and the central hadron calorimeter $(\mathrm{CHC})$ covering $\pm 5 \mathrm{mrad}$ around the beam line direction. The main hadron calorimeter (HC) is an iron-gas sampling device with tower read out geometry consisting of 28 alternating layers of iron absorber ( 8 interaction lengths) and gas/wire read out layers operated in a 50/50 mixture of Argon-Ethane gas, and instrumented with anode pads to detect ionization from shower particles. The CHC detector is a uranium-scintillator calorimeter (6.4 interaction lengths) readout by two

\footnotetext{
"The fire in 1987 forced us to rebuild the IE, which was then made of fibers gathered in strips.
} 
photomultiplier tubes. The main purpose of hadron calorimetry was to reject electromagnetic events while being a part of the second level trigger.

\subsubsection{Trigger}

The major background in photoproduction experiments comes from the high rate of $e^{+} e^{-}$pair production relative to the number of hadronic interactions ( $~ 500: 1$ in E687). In order to effectively suppress electromagnetic events, the trigger is organized in two levels. The first level trigger (MASTER GATE) is a simple interaction trigger requiring that an interaction had occurred in the experimental target caused by a neutral particle and that at least two charged particles were accepted by the detector. The second level trigger operates on signals latched during the first level trigger duration and is designed to reject electromagnetic interactions and insure that the incoming photon had a high enough energy.

\section{First Level Trigger}

The MASTER GATE uses scintillation trigger counters to signify the presence of charged particles. These counters cover the whole region of the geometric acceptance of the detector and are divided in three logical categories: the VETO counters, experimental target counters, and geometrical acceptance counters. The VETO ensured that charged particles do not enter the experimental target nor the detector from the direction of the photon beam during the event. It consisted of two parts, AO a scintillator paddle in the beam line in front of the experimental target and sets of large counters labeled TM1, TM2, AM1 and AM2 covering the rest of the beam line area to screen for albedo associated with the beam transport system. Experimental target area counters TR1 and TR2, positioned before and after the microstrip detector, insured that charged particles emerging from the target were accepted by the microstrip system. Presence of a charged particle within the outer region of the 
detector, was indicated by the outer hodoscope $(\mathrm{OH})$ placed in front of the outer electromagnetic calorimeter. Acceptance of the charged particles within the inner region of the detector was signaled by a set of horizontal and vertical hodoscopes $(\mathrm{H} \times \mathrm{V})$ located at the back of the last PWC station.

The MASTER GATE was formed from signals of all these counters requiring that no charged particle comes from upstream of the target, a charged particle passes through the microstrip detector, and at least two charged particles are accepted by the rest of the detector with either both being in the inner region of the detector or one being in the outer and another in the inner region of the detector. During the run the typical MASTER GATE rate was $50 \mathrm{kHz}$.

\section{Second Level Trigger}

The second level trigger was constructed from programmable trigger modules [Ste 87]. Each of the trigger modules was designed according to a particlular physics goal. The physics requrements triggered on included charged particle multiplicity, presence of a muon, unbiased data, and a certain amount of observed hadronic energy. The logical .OR. of all trigger modules constituted the second level trigger. The primary data taking trigger module required:

- More than one hit in the first PWC station outside the $e^{+} e^{-}$region;

- $E_{\text {total }}>40 \mathrm{GeV}$ for the energy detected by the Hadron calorimeter;

- A valid RESH response corresponding to $E_{\gamma}>110 \mathrm{GeV}$.

The data acquisition is disabled for a $1.2 \mu$ s interval needed by the second level electronics to make a level-two trigger decision. If the trigger is not satisfied, a clear cycle is initiated which takes about $1 \mu \mathrm{s}$. So the dead time generated for each MASER GATE which fails to produce a second level trigger is $2.2 \mu \mathrm{s}$. If the second level trigger is satisfied, a full read out and automatic clear are initiated. 


\subsubsection{Data Acquisition}

The data acquisition system was custom designed for the experiment by the Fermilab computer group [Pet 90]. It was designed to efficiently store events (typical size 6 Kbytes) onto permanent media and be able to keep up with the readout rate of the digitizing electronics. This system was able to record 10000 events per minute. In order to accomplish this task, all $\approx 30000$ electronic channels were divided into five data streams, each ending with a LeCroy 1892 buffer memory located in the FASTBUS crate. From these memories data was transferred into $\mathrm{ACP}^{5}$ nodes located in a VME crate. From these nodes the data were transfered to four exabyte $8 \mathrm{~mm}$ drives connected in parallel.

The acquisition of data begins as soon as the second level trigger has been satisfied. First a $1.4 \mathrm{~ms}$ dead time is initiated to prevent the pile up of events while the data from an accepted event is being processed. During this time the data are digitized and read into five memory buffers (having a total of $18 \mathrm{Mbytes}$ of available memory). This intermediate step in data recording was introduced in order to take advantage of the accelerator $\mathrm{cycl}^{6} \mathrm{e}$ while writing to slow media ( $8 \mathrm{~mm}$ tape drives are capable of processing only $250 \mathrm{Kbytes} / \mathrm{sec}$ ).

During the runs in 1990 and 1991 we accumulated $5 \times 10^{8}$ standard triggers that were stored on one thousand $8 \mathrm{~mm}$ tapes.

\footnotetext{
${ }^{5}$ ACP stands for Advanced Computer Project and represents parallel network of model 68020 processors developed by the Fermilab computing department.

${ }^{6}$ The beam was available to the users for $20 \mathrm{sec}$ during every minute.
} 


\section{CHAPTER 3}

\section{Data Analysis}

This chapter describes the process of data analysis starting with the interpretation of the information recorded on tape and concluding with methods used to extract particular charm candidate samples. The whole data set consisted of $\sim 5 \times 10^{8}$ recorded events corresponding to $\sim 3 \times 10^{12}$ bytes recorded on $8 \mathrm{~mm}$ tape media. The information recorded on tape consisted of hits/pulseheights observed by various elements in the detector. In order to efficiently process this large data set by reconstructing the kinematic as well as dynamical properties of the events and to extract particular charm candidates, the analysis was divided into three logical stages: data reconstruction (PASS1), data reduction (SKIM), and particular signal extraction. It is not difficult to conclude that first two tasks were group efforts requiring many hours/days of computer time as well as careful monitoring of the whole process. The first two stages were accomplished at Fermilab using a cluster of RISC workstations dedicated to this project from Fall 1991 to Fall 1992.

\subsection{Data Reconstruction}

The reconstruction stage was implemented in the form of a package of routines called PASS1 designed to reconstruct the charged particle trajectories, their momenta, their identity, neutral vees, primary and secondary vertices, neutrals and their corresponding energy from the hit information stored on tape and a database of parameters 
characterizing each detector element.

In order. to extract charm signals for the lifetime analysis only information pertinent to charged particles was utilized. This included their trajectories, momenta and their identity as given by the Cerenkov system. The algorithms that provided this information will be described in the sections to follow.

\subsubsection{Charged Particle Tracking}

Charged particle trajectories were detected by two independent detector subsystems described in the previous chapter as microstrip (SSD) and multi-wire proportional chamber (PWC) systems. The responses of these two subsystems to charged particles were first analyzed independentl ${ }^{1} y$, and at a latter stage the fitted track segments were linked where possible to yield the complete trajectory of the charged particle throughout the apparatus.

\section{Microstrip Reconstruction}

The microstrip track reconstruction package [Mor 88] (MICRORICO) was functionally divided into three parts: hit analysis, projection pattern recognition, and three dimensional space track fitting.

An analysis of valid hits was performed on the basis of observed charge in order to assign optimal coordinate positions to the track intersection points with the struck planes and to resolve single and multiple track crossings in the case of adjacent hits. In the case of isolated hits the centroid of the hit strip was used as an intersection point. For two adjacent hits with total observed charge consistent with a single minimum ionizing particle (MIP) crossing, the centerline between the strips was used as the coordinate. For three hit clusters with accumulated charge consistent with two MIP

\footnotetext{
${ }^{1}$ One notable exception exists and will be discussed in conjunction with PWC reconstruction.
} 
crossings, either the two centerlines between the strips were used, or the center line between the two and a centroid of the third strip were used.

The next step involved the formation of track projections along the three axes from the previously determined spatial coordinates. Each projection was required to have at least three out of four possible hits and to pass a loose $\chi^{2}$ test $^{2}$ on a least squares fit to the hits. The weight associated with each hit in the fit procedure is always $\sqrt{12}$ /pitch. Sharing of hits among different tracks was permitted for all combinations except for hits in the third and fourth station that already belonged to a projection which had four hits.

Three dimensional space tracks are formed by matching all newly found projections in space. The matching was done by grouping two projections and then searching for the third one to complete a space point triplet. This was accomplished by computing the intersection points at the first and last planes between the two chosen projections and imposing a maximum separation cut on the coordinates of the third projection. The twelve coordinate positions associated with four grouped triplets were subject to a least squares fit. The $\chi^{2}$ value per degree of freedom for final candidates was required to be lower than eight. Tracks sharing one or two projections are then arbitrated on the basis of their $\chi^{2}$ value, and clusters of closely spaced tracks were combined into a single track if possible. Hits not associated with any reconstructed space track are used to search for wide angle tracks or single segments of highly multiple coulomb scattered tracks.

The efficiency of this algorithm to find tracks has been evaluated by a full Monte Carlo simulation of the microstrip system, including hot strips, dead strips and multiple Coulomb scattering effects to be $96 \%$. The number of fake tracks (drawn from accidental association of hits) was found to be less than $3 \%$.

\footnotetext{
${ }^{2} \chi^{2} \leq 30$ for 4 point tracks and $\chi^{2} \leq 20$ for 3 point tracks.
} 
This routine operated on the raw hits without the benefit of the downstream tracking information implying that no knowledge of the track momentum was utilized during the fitting procedure thus ignoring the effects of multiple coulomb scattering (MCS). The MCS effect is an important factor due to the amount of material introduced by the silicon planes themselves, and could cause loss in efficiency if relatively loose cuts were not imposed. However, during the later stages of the analysis, (for example when computing vertex positions), accurate knowledge of track covariance matrices becomes important. The resolution of the track is expressed as the error in the $x-y$ plane extrapolated to a particular $z$ location. For tracks consisting only of hits from the high resolution region of the microstrips, the resolution extrapolated to the center of the target is calculated to be

$$
\begin{aligned}
& \sigma_{\mathrm{x}}=11 \mu \mathrm{m} \sqrt{1+\left(\frac{17.5 \mathrm{GeV}}{p}\right)^{2}} \\
& \sigma_{\mathrm{y}}=7.7 \mu \mathrm{m} \sqrt{1+\left(\frac{25.0 \mathrm{GeV}}{p}\right)^{2}}
\end{aligned}
$$

The second term under the square root represents the multiple coulomb scattering contribution to the resolution. These formulae were checked against data by comparing track miss distances to the vertex of origin with the anticipated errors. The distribution of miss distances divided by the anticipated error could be fit with a Gaussian function having nearly unit width, indicating correct error estimation.

\section{PWC Reconstruction}

The algorithm designed to form tracks in the PWC system utilized hit information from twenty planes divided among four views. It was designed to reconstruct tracks 
with hits in 3, 4 or 5 PWC stations. In each case a track is required to have hits in the first PWC station. This algorithm is similar in design to the microstrip tracking algorithm described in the previous section in that it first forms projections from hits in all four views. The projections in the $\mathrm{Y}, \mathrm{U}$ and $\mathrm{V}$ views are formed from $\mathrm{PWC}$ information alone while the non-bend $\mathrm{X}$-view projections are made by searching for PWC hits which match the microstrip track extensions. Tracks are formed by matching all four projections. After all the tracks having microstrip-extended projections are found, additional $\mathrm{X}$ projections (if any) are constructed from the remaining PWC hits in the $\mathrm{X}$-view and the matching procedure with other $\mathrm{Y}, \mathrm{U}$ and $\mathrm{V}$ projections is repeated.

A least squares fit is performed on all candidate tracks. The fit parameters are the intercepts and slopes of the track in both $\mathrm{X}$ and $\mathrm{Y}$ views at the second magnet (M2) bend plane, and in the case of 4 or 5 station tracks, the change in slope in the $\mathrm{Y}$ view (bend angle) between the track segments upstream and downstream of M2. A $\chi^{2}$ per degree of freedom cut is applied to each track in order to pass the fit. Tracks were not permitted to have more than four missing hits among all planes nor more than two missing hits in any one station. As before, arbitration between tracks with shared projections had to be performed. In addition there were various magnetic corrections due to the finite lengths of the magnets, existence of so called off-field ${ }^{3}$ components and the existence of fringe fields outside the magnets. All these effects were treated as higher order corrections to the linear least squares fit described above.

In addition to the main algorithm, several additional tracking routines were designed to recover track topologies that were missed. These included tracks with hits only in the first two PWC stations P0 and P1, and three-station extensions in P3 and P4.

\footnotetext{
${ }^{3} \mathrm{By}$ off-field we mean magnetic field components along directions other than the direction of the main field $\left(B_{x}\right)$.
} 
The overall efficiency for track reconstruction was determined to be greater than $98 \%$ for tracks exceeding $5 \mathrm{GeV} / \mathrm{c}$ by using Monte Carlo generated tracks ${ }^{4}$. Spurious tracks were found less than $1 \%$ of the time. In order to reduce CPU time consumption, the total number of reconstructed tracks per event was limited to 30. Events with high apparent multiplicity usually resulted from electrical oscillations in the PWC system.

\section{Linking of SSD and PWC Tracks}

It is important to match track segments between the two tracking systems since the microstrip tracks have a high spatial resolution but no momentum or identification information and conversely PWC tracks have a lower spatial resolution but have momentum and identification information available. A knowledge of the track momentum is essential for correct calculation of the vertex resolution, which has a direct influence on the ability to resolve spatially separated vertices (main signature of charm decays). On the other hand tracks that are not linked are possibly indications of neutral particle decays downstream of the microstrip system.

The linking was performed by comparing all possible combinations of tracks from the two systems and projecting them to the bend plane of the first magnet. At this point the difference between the slopes and intercepts (for $x$ and $y$ views) of the two tracks were computed. Combinations falling within a specified window were subjected to a global least squares fit using all the hits from both systems. Five station PWC tracks were required to have consistent bend angles in two magnets. The algorithm allowed one microstrip track to link to at most two PWC tracks; conversely a PWC track could link to only one microstrip track. This allowed for proper linkage of Bethe-Heitler pairs created in the target or microstrip detectors.

\footnotetext{
${ }^{4}$ Individual plane efficiencies were simulated
} 
The linking efficiency as a function of the track momentum has been measured by projecting the PWC tracks towards the SSD vertex, and then checking to see if they are linked. The efficiency at high momentum $(\mathrm{P}>50 \mathrm{GeV} / \mathrm{c})$ is found to be $91.4 \%$ for 3 -station and $97.0 \%$ for 5 -station tracks. The efficiency is reduced at lower momentum due to the multiple coulomb scattering within the microstrip system which causes tracks not to be found by the SSD algorithm.

To improve the linking efficiency, a special recovery program was written to look for missing microstrip tracks by extending unlinked PWC tracks back into the microstrip system in search for possible matches with unused hits. This increased the linking efficiency of 3 and 5 -station tracks by $2.5 \%$ and $1.5 \%$ respectively.

An alternative approach to measure the linking efficiency as a function of track momentum was performed by reconstructing $\phi$ meson decays into $K^{+} K^{-}$. The narrow width of the $\phi$ resonance and the requirement that the two tracks of opposite charge be identified as kaons by the Čerenkov system was sufficient for clear reconstruction of the $\phi$. The significance of signal was further improved by requiring that one of the two tracks be linked. Utilizing the prominent $\phi$ resonance obtained in this manner, the linking efficiency was calculated as the ratio of detected $\phi$ mesons with the linking requirement imposed on both tracks to the yield obtained by requiring only one of the tracks to be linked. Fig. 3.1 shows linking efficiency measured by this method in cases of 3 and 5-station tracks. To obtain the yield, the data was fit with a Pwave Breit-Wigner function [Jdj 64] and a first order polynomial. This technique will be utilized again in the following section in order to measure the kaon identification efficiency as a function of momentum.

\subsubsection{Momentum Determination}

The momenta of charged particles are determined by the amount of deflection in the known magnetic fields of first and second analysis magnets. Depending on the track 

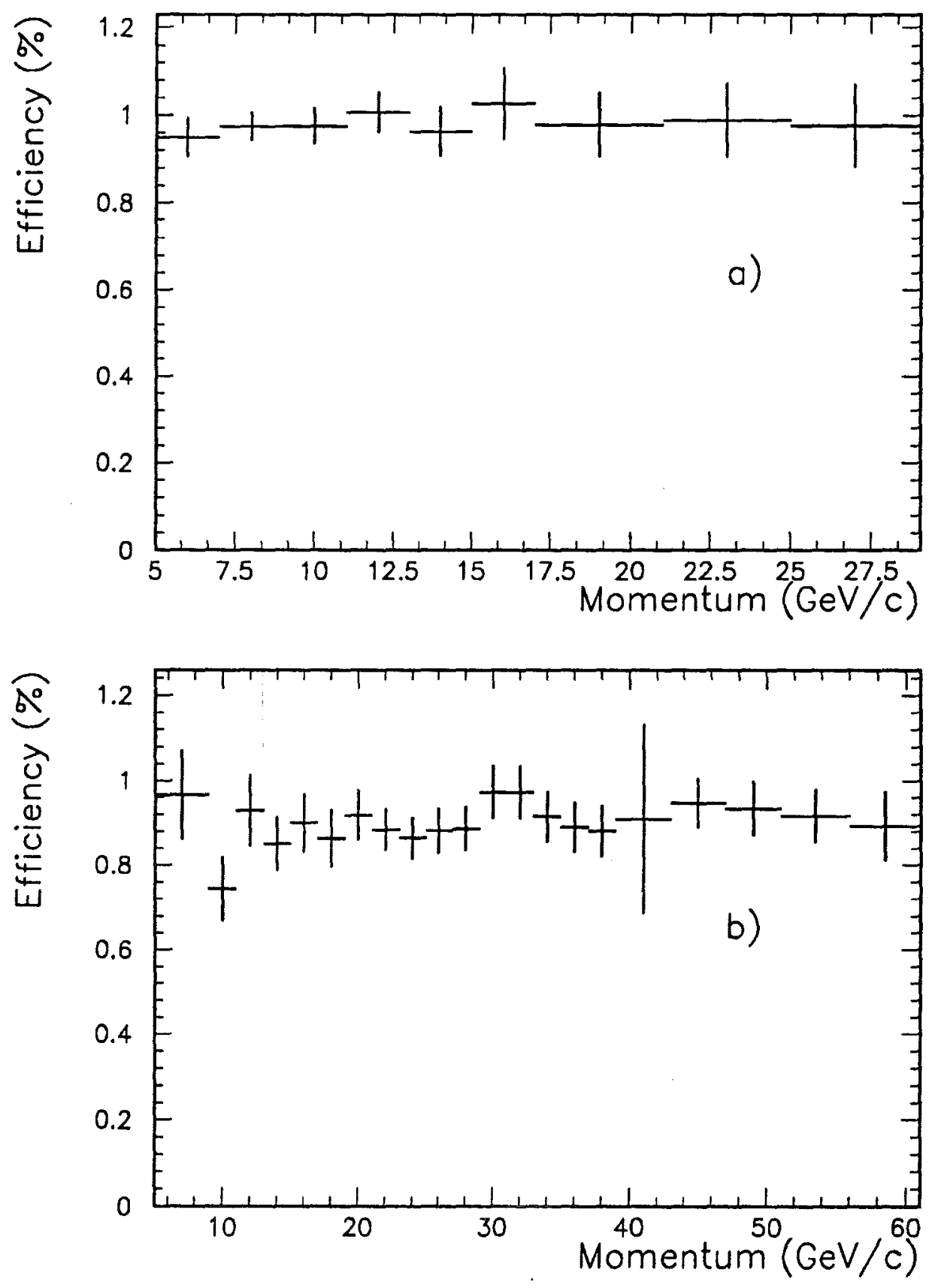

Figure 3.1. Linking efficiency of kaons as a function of momentum for: a) 3-station tracks; b) 5-station tracks. 
topology (type) its momentum is determined either by M1 or M2 or by both. In case of unlinked 3 -station tracks it is necessary to assume that the track came from the best vertex ${ }^{5}$ formed by microstrip tracks. The anticipated [Jim 88] momentum resolution is computed from the spectrometer layout with MCS effects included to be

$$
\frac{\sigma}{P}=3.4 \%\left(\frac{P}{100 \mathrm{GeV} / \mathrm{c}}\right) \sqrt{1+\left(\frac{17 G e V}{P}\right)^{2}} \text { for } \mathrm{M} 1
$$

and

$$
\frac{\sigma}{P}=1.4 \%\left(\frac{P}{100 G e V / c}\right) \sqrt{1+\left(\frac{23 G e V}{P}\right)^{2}} \text { for M2. }
$$

These resolutions were verified by comparing track momenta measured by the two magnets independently. A fit to the error normalized difference of the two measurements with a Gaussian function shows that the resolution is known to within $10 \%$.

\subsubsection{Particle Identification by the Ćerenkov system}

The algorithm designed to provide particle identification from the three threshold Cerenkov counters utilized the pulse height information from 300 cells recorded on tape and a table of parameters specifying counter thresholds, geometry and mean photoelectron yield for each cell.

Reconstructed charged tracks traversing the counters are categorized as either electrons, pions, kaons or protons characterized by a particular threshold momentum (see Table 2.6) above which Čerenkov light is emitted (muons are to close enough in mass to be categorized as pions). Using the known momentum of a given track and the properties of the gas in each counter, a prediction could be made concerning

\footnotetext{
${ }^{5}$ In lieu of an SSD vertex a PWC vertex is used, barring that the center of the target is chosen.
} 
the light deposition in the counters for each of the four assumed identities. On the other hand the actual response of the counters (on/off) to a given track can support or contradict any given hypothesis and thus include or exclude certain categories as possible identities. Depending on the particle momentum and on the extent that the three counters agree on the allowed categories, the track may be given a definite identity, or suffer from some ambiguity.

The analysis begins by comparing the pulse height from each cell with a threshold in order to determine its ON/OFF status. The value of the threshold typically amounted to several $\mathrm{pC}$ above the pedestal in order to reduce the frequency of accidentals. In the next step a given track is projected through each of the three counters to determine the relevant cells. Relevant cells included the cell struck by the track and all adjacent cells. The amount of light expected in a given cell depends on the particle hypotheses, track momentum, and the overlap of the Čerenkov cone with the cell area. An anticipated light yield based on mean photoelectron yield obtained from counter calibration is calculated for each of the relevant cells for pion and electron hypotheses depending on the track momentum. The analysis is then iterated to choose the better assumption. If the observed pulse height was larger than the threshold for the cell predicted to detect light from a given track, then the counter is called "ON" for that track. In cases where more than one track could possibly contribute to the relevant cell or cells the counter is called "CONFUSED" for that track. The counter is called "OFF" when a reasonable amount of light is expected in the relevant cells but the cells were off.

The determined status (ON,OFF or CONFUSED) of each counter is compared to the track momentum and the counter's threshold for a given particle category. The track momentum is assigned to one of seven momentum regions of the given counter. Four momentum regions come from pion, kaon and proton thresholds while the other three are the transition regions. Transition regions exist due to the fact that the 
Table 3.1. Values assigned to Cerenkov ISTATP variable

\begin{tabular}{|c|c|}
\hline ISTATP & Identification \\
\hline \hline 0 & Inconsistent information \\
\hline 1 & $e^{ \pm}$ \\
\hline 2 & $\pi^{ \pm}$ \\
\hline 3 & $e^{ \pm}$or $\pi^{ \pm}$ \\
\hline 4 & $K^{ \pm}$ \\
\hline 7 & $e^{ \pm}, \pi^{ \pm}$or $K^{ \pm}$ \\
\hline 8 & $p^{ \pm}$ \\
\hline 12 & $K^{ \pm}$or $p^{ \pm}$ \\
\hline 14 & $\pi^{ \pm}, K^{ \pm}$or $p^{ \pm}$ \\
\hline 15 & $e^{ \pm}, \pi^{ \pm}, K^{ \pm}$or $p^{ \pm}$ \\
\hline
\end{tabular}

counter response is not a step function but rather a gradual increase in light yield above the threshold. Monte Carlo studies show that these regions are narrow and extend from a given threshold to 1.08 times the given threshold.

Using the above information a four bit status word is set for each counter. Each bit location is associated with one of the particle types starting from the least significant bit:

- Bit 1 : Electron status

- Bit 2 : Pion status

- Bit 3 : Kaon status

- Bit 4 : Proton status

A bit is set on if the hypothesis is satisfied and off if it is contradicted. These 4-bit status words for each of the three counters are logically AND-ed to form the Cerenkov identification variable, ISTATP. Table 3.1 lists the possible values and interpretations of the ISTATP variable. Fig. 3.2 shows the momentum ranges where certain identification classes are possible for 3 -station and 5-station tracks. 

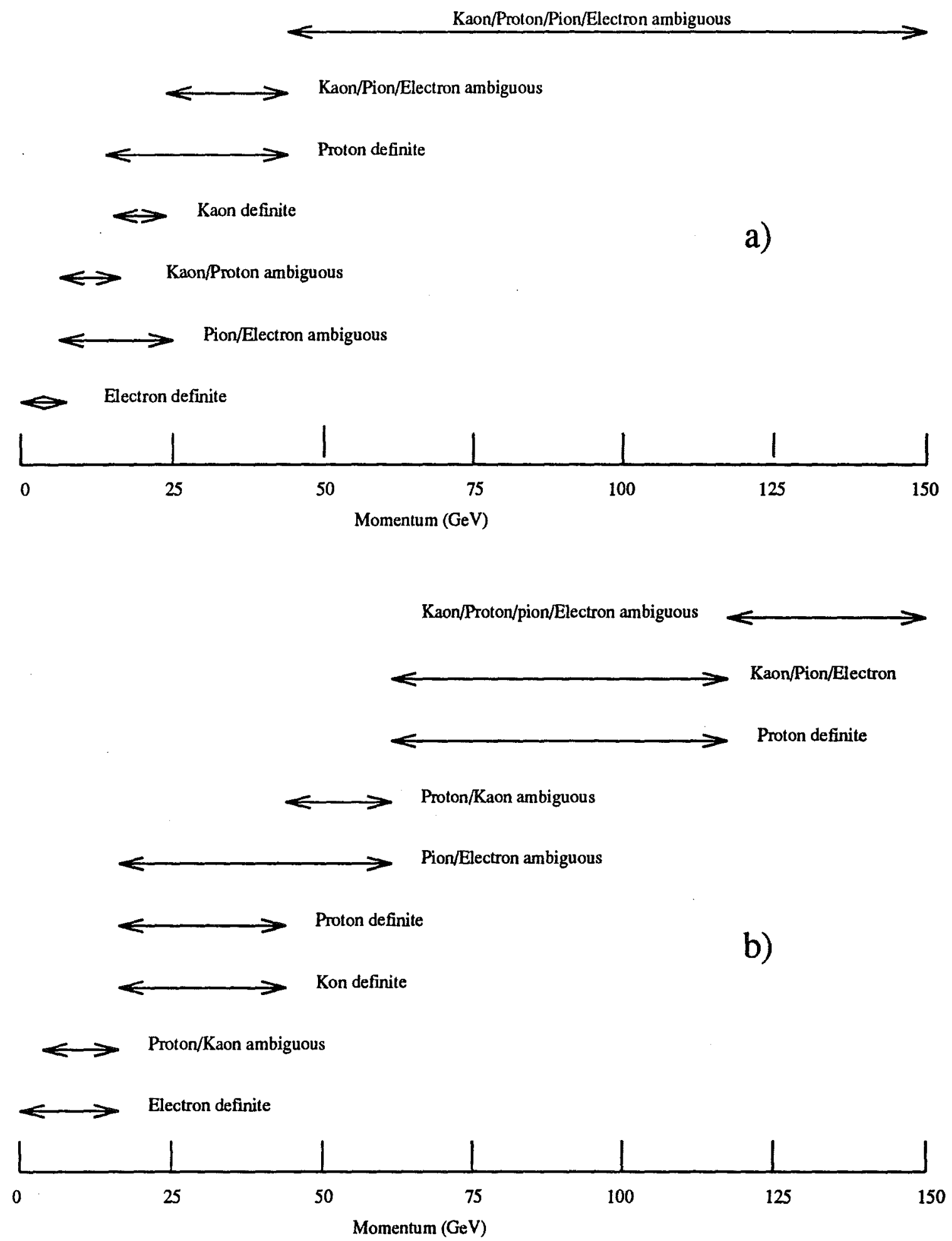

Figure 3.2. Momentum ranges where certain identification classes are possible: a) 3-station tracks; b) 5-station tracks. 
The performance of the Cerenkov algorithm and counter system was studied using decays whose observation did not solely depend on the particle identification. These decays included:

$$
\begin{aligned}
& \text { - } \phi^{\prime \prime} \rightarrow K^{+}+K^{-} \\
& \text {- } K^{* 0} \rightarrow K^{+}+\pi^{-} \\
& \text {- } K_{s}^{0} \rightarrow \pi^{+}+\pi^{-} \\
& \text {- } \Lambda^{0} \rightarrow p+\pi^{-}
\end{aligned}
$$

Another advantage of using these decays was their copious yields allowing for very high statistics studies. The efficiency studies were accomplished by comparing the yields of a given signal before and after the Čerenkov requirement. These studies were also performed with much lower statistics D samples obtained by topological analysis of events only. The problem then arises that global identification efficiencies so obtained may not be the same for $\phi^{0}$ and $D$ mesons due to the different momentum distributions of kaons coming from these decays. In order to measure the kaon identification efficiency as a function of track momentum a special routine was designed to select candidate $\phi^{0}$ decays to $K^{+} K^{-}$with a Čerenkov requirement imposed on only one of the kaons. The data was binned in several momentum bins and efficiency was determined as the ratio of yields before and after imposing the Čerenkov requirement on the second track forming the $\phi^{0}$ candidate. The measured kaon identification efficiency is shown in Fig. 3.3 for kaons defined as tracks with Čerenkov variable ISTATP $=4,12$. Analogous study has been performed with $K_{s}^{0}$ to measure the pion identification efficiency. These measured efficiencies were compared to predicted Monte Carlo efficiencies showing a good agreement. 

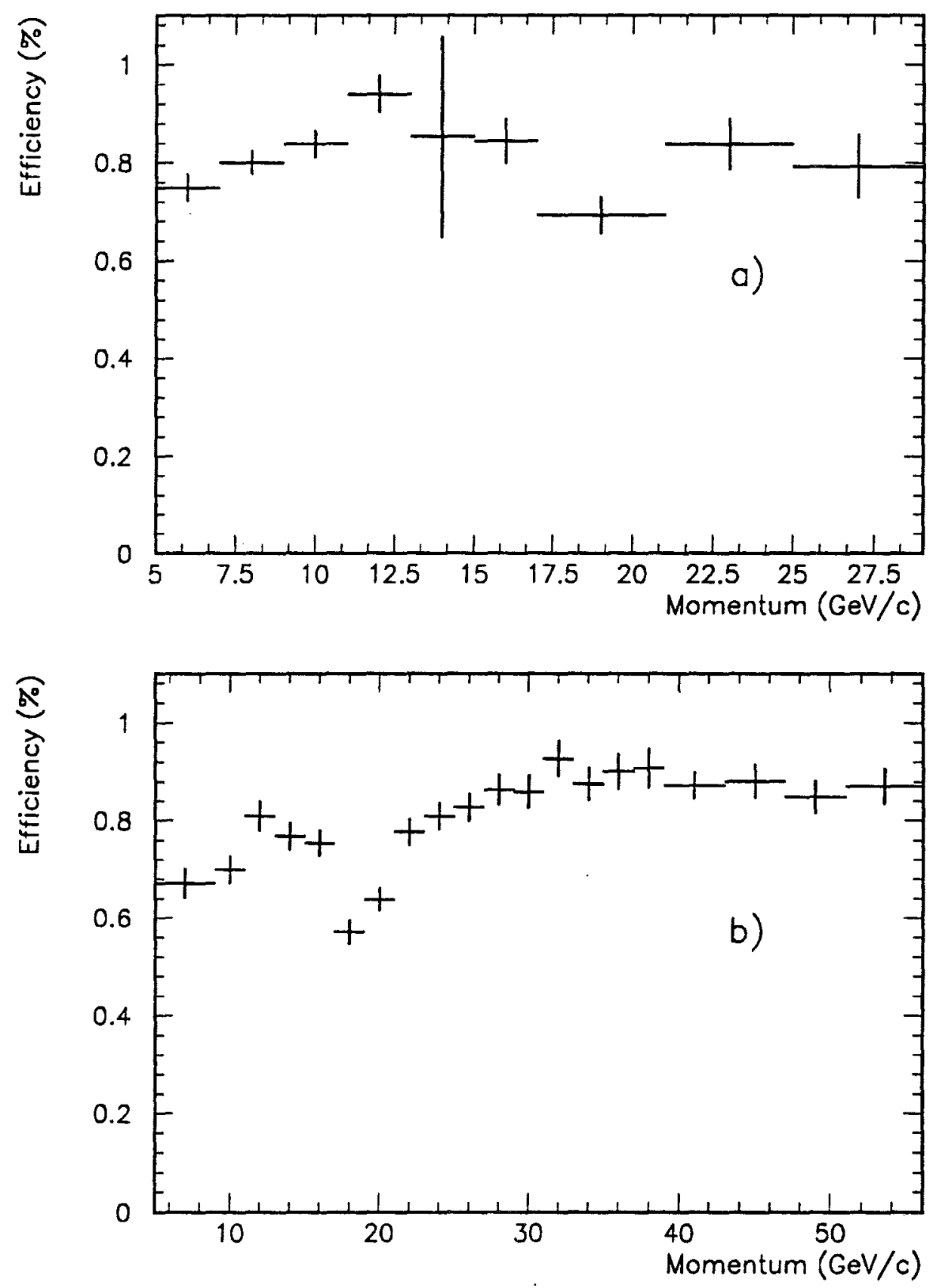

Figure 3.3. Kaon ID efficiency as a function of momentum for: a) 3-station tracks; b) 5-station tracks. 


\subsection{Data Reduction}

The first stage of data analysis (PASS1) produced close to two thousand $8 \mathrm{~mm}$ tapes amounting to about $4 \times 10^{12}$ bytes. In order to reduce this large data set to a more manageable size a separate package of routines called SKIM was designed and run over the output of the PASS1. The SKIM package consisted of several independent subroutines carefully designed to select particular final states. Table 3.2 displays the lists of implemented event selection strategies.

The work presented in this thesis is based on the data selected by the $D$ meson and $\phi^{0}$ meson skims (skim bits 9 and 28 respectively).

\subsection{1 $\phi^{0}$ Meson Skim}

This algorithm was designed to select $\phi^{0}$ meson decays to $K^{+} K^{-}$. The algorithm first checked that an event had more than one track identified by the Cerenkov system as being kaon consistent (ISTATP $=4,12$ and 7 if the momentum of the candidate is greater than $61.5 \mathrm{GeV} / \mathrm{c}$ ), and that all kaon candidates were linked between the microstrip and PWC systems. Each pair of opposite charge tracks from this set was combined to form a vertex and the corresponding invariant mass was calculated. Events that had at least one combination with invariant mass $M_{K^{+} K^{-}}<1.1 \mathrm{GeV} / c^{2}$ and whose confidance level on the hypothesis that the two tracks came from a common vertex exceeded $1 \%$ were selected.

Although limited in scope this algorithm had an advantage of not having a requirement on the existence of two separate vertices thus allowing for studies involving neutral and missing particles where the secondary vertex is not well measured. It was also an efficient skimming algorithm selecting only $1 \%$ of all recorded events. The resulting mass plot is shown in Fig. 3.4 displaying an impressive $\phi^{0}$ resonance peak. A fit with a $\mathrm{P}$-wave Breit-Wigner function [Jdj 64] yielded over $10^{6}$ reconstructed $\phi^{0}$ meson 
Table 3.2. List of skimming strategies

\begin{tabular}{|c|l|}
\hline Skim Bit & Skim Goal \\
\hline 1 & Semileptonic skim \\
2 & $1 \%$ of all events \\
3 & Prescaled Unbiased Data \\
4 & $e^{+} e^{-}$pair trigger \\
6 & Two vertex skim \\
7 & Cascade skim \\
8 & $K_{s}$ skim \\
9 & D meson skim \\
10 & D meson skim ( $D^{*}$ flag set) \\
11 & D meson skim (Golden Mode flag) \\
12 & \\
13 & $\Lambda^{0}$ skim \\
14 & Kink skim \\
15 & Charm Baryon skim \\
16 & $\Lambda_{c} \rightarrow P K \pi$ skim \\
17 & Leptonic skim \\
18 & $\mu$ skim for $J / \Psi$ \\
19 & Diffractive skim \\
20 & \\
21 & Topological skim 1 \\
22 & Topological skim 2 \\
23 & \\
24 & \\
25 & Kaon lepton skim \\
26 & Kaon id skim \\
27 & Kaon linking skim \\
28 & $\phi^{0}$ meson skim \\
29 & prescaled bit 26 \\
30 & prescaled bit 27 \\
\hline
\end{tabular}




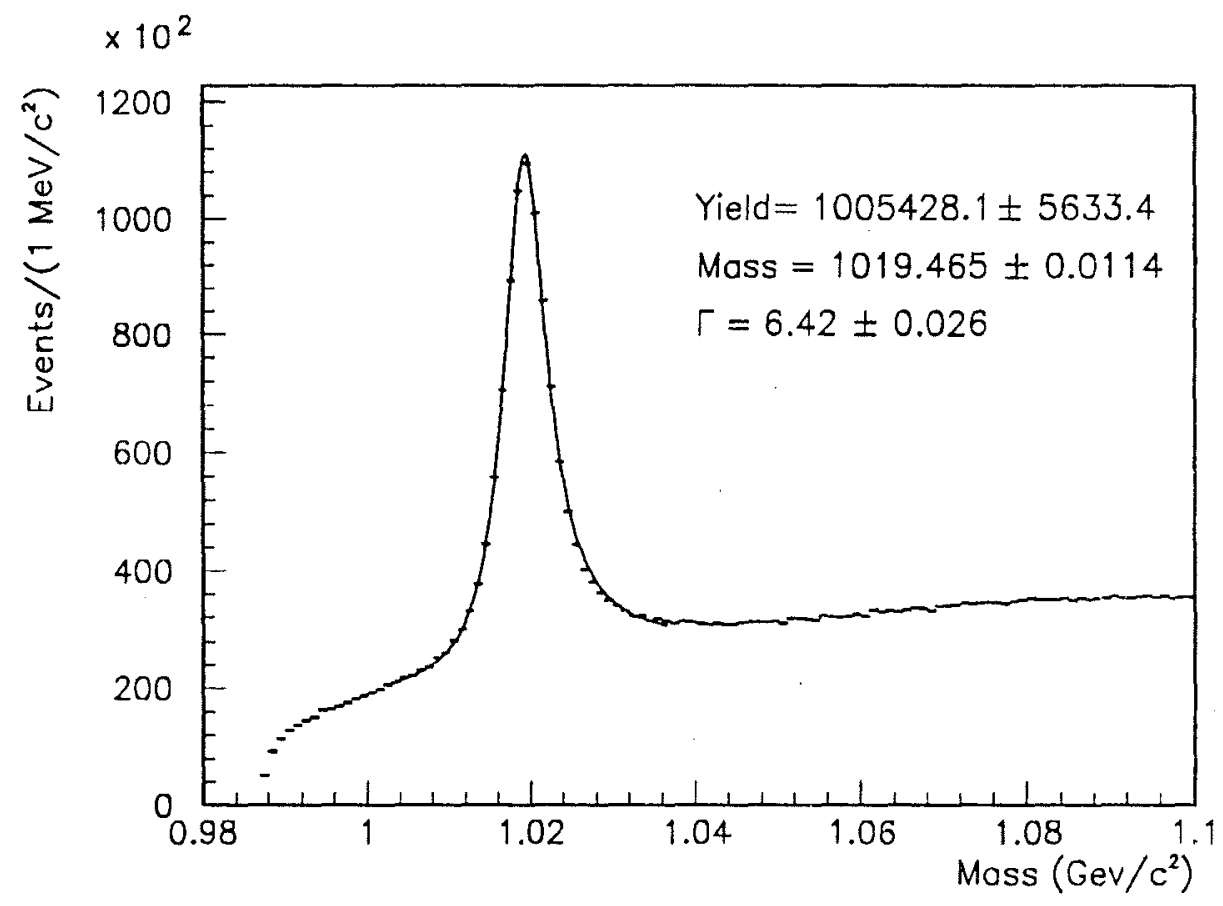

Figure 3.4. $K^{+} K^{-}$invariant mass distribution

decays with a mean mass $M_{\phi}=1019.47 \pm 0.01 \mathrm{MeV} / c^{2}$ and width ${ }^{6} \Gamma_{\phi}=6.42 \pm 0.03$ $\mathrm{MeV} / \mathrm{c}^{2}$. This plot demonstrates the power of the constraint on the $\phi^{0}$ mass which will be exploited later to isolate a very clean $D_{s}^{ \pm}$signal.

\subsubsection{Meson Skim}

The $D$ meson skim [Jar 90] was the most comprehensive of the algorithms designed to search for well over 100 charged decay modes of the $D^{0}, D^{ \pm}, D_{s}^{ \pm}$and $D^{* \pm}$ mesons. The decay modes included $\pi^{ \pm}, K^{ \pm}$and $K_{s}$ mesons in combinations up to 7 bodies.

In order to suppress the large attendant combinatoric backgrounds the algorithm relied on Cerenkov particle identification and the ability of the microstrip system to separate primary and secondary vertices. Tracks were first classified as pions or kaons

\footnotetext{
${ }^{6}$ The width $\Gamma_{\phi}$ is a convolution of the natural $\phi$ width and the invariant mass resolution $\sigma_{m} \approx 2$ Mev $/ c^{2}$ of the $K^{+} K^{-}$system in this spectrometer.
} 
based on Cerenkov particle identification. These tracks were then combined to form appropriate charm decay candidates. Each of these combinations is then passed to the "candidate driven" vertexing algorithm [Cul 91a] that attempts to form a charm vertex from the candidate tracks. If successful it then attempts to form a primary vertex around the charm vector. By using the charm vector as a seed, this algorithm is able to find single track primary vertices. The quality of the formed vertex is measured by the confidence level of the hypothesis that considered tracks emerged from a common vertex. To qualify as valid vertices, primary and secondary vertices were required to have confidence levels greater than $0.1 \%$. The low confidence levels were chosen in order to minimally bias the skimmed data sample. Upon successful formation of the primary and secondary vertices the algorithm calculates the significance of separation $\ell / \sigma_{\ell}$ of the two vertices defined as the distance $\ell$ between the two divided by the error on this distance $\sigma_{\ell}$. The distinctive topology of charm events (i.e., the existence of two spatially separated vertices) provides the single most powerful tool to isolate charm signals from non-charm background.

The $D^{*}$ candidates were formed from existing $D^{0}$ candidates by calculating an invariant mass with a cascade pion candidate selected from the set of unused tracks. The candidates were then selected based on the value of the $D^{*}-D^{0}$ mass difference. The events were selected if at least one of the candidate combinations satisfied a set of criteria specified for a given decay. The cuts are optimized with a goal to keep the efficiency for charm detection high while reducing the whole data set to a manageable size. The $D$ skim kept $15 \%$ of raw events recorded on tape.

The samples for the decays of $D^{0} \rightarrow K^{-} \pi^{+}, D^{0} \rightarrow K^{-} \pi^{+} \pi^{-} \pi^{+}$, and $D^{+} \rightarrow$ $K^{-} \pi^{+} \pi^{+}$and their charge conjugates were obtained from the pool of events selected by this algorithm. The $D^{0}$ candidates are searched for as a direct product of the photon-Be interactions and as one of the two decay products of the $D^{* \pm} 7$. Table 3.3

\footnotetext{
${ }^{7}$ These two sets overlapped but were not identical since the requirement on the mass difference in
} 
Table 3.3. $D$ meson skim selection criteria

\begin{tabular}{|l|c|c|c|c|c|c|}
\hline Decay Mode & $\begin{array}{c}\text { ID } \\
K, \pi\end{array}$ & $\begin{array}{c}\text { Primary } \\
\text { Vtx. CL }\end{array}$ & $\begin{array}{c}\text { Charm } \\
\text { Vtx. CL }\end{array}$ & $\begin{array}{c}\ell / \sigma_{\ell} \\
\left(\mathrm{GeV} / c^{2}\right)\end{array}$ & $\begin{array}{c}m_{D^{*}-m_{D}} \\
\left(\mathrm{GeV} / c^{2}\right)\end{array}$ \\
\hline \hline$D^{0} \rightarrow K^{-} \pi^{+}$ & $\mathrm{KP} 7,-$ & 0.001 & 0.001 & 2 & $1.665-2.05$ & - \\
\hline $\begin{array}{l}D^{*+} \rightarrow \pi^{+} D^{0}, \\
D^{0} \rightarrow K^{-} \pi^{+}\end{array}$ & $\mathrm{KP} 7,-$ & 0.001 & 0.001 & - & $1.665-2.05$ & $<0.16$ \\
\hline$D^{0} \rightarrow K^{-} \pi^{+} \pi^{-} \pi^{+}$ & $\mathrm{KP} 7,-$ & 0.001 & 0.001 & 2 & $1.665-2.05$ & - \\
\hline $\begin{array}{l}D^{*+} \rightarrow \pi^{+} D^{0}, \\
D^{0} \rightarrow K^{-} \pi^{+} \pi^{-} \pi^{+}\end{array}$ & $\mathrm{KP} 7,-$ & 0.001 & 0.001 & - & $1.665-2.05$ & $<0.16$ \\
\hline$D^{+} \rightarrow K^{-} \pi^{+} \pi^{+}$ & $\mathrm{KP} 7,-$ & 0.001 & 0.001 & 2 & $1.665-2.15$ & - \\
\hline
\end{tabular}

lists the cuts employed to select these five decay channels from the raw data by the $D$ skim. The requirement commonly referred to as KP7, corresponds to Čerenkov identification of kaons representing a logical $O R$ between ISTATP values 4, 12 and 7 if the track momentum is greater than $61.5 \mathrm{GeV} / \mathrm{c}$ (see Table 3.1).

\subsection{The Final Event Selection}

The events selected by the two skimming procedures described in the previous section were analyzed for decay modes suitable for the study of $D^{0}, D^{ \pm}$and $D_{s}^{ \pm}$lifetimes. These decay modes included:

- $K \pi, K \pi \pi \pi$ of the $D^{0}$

- $K \pi \pi$ of the $D^{+}$

- $\phi \pi$ of the $D_{s}$

In order to produce a clean and unbiased but still large charm sample the final selection procedure included additional requirements. The available background reduction tools included:

the case of the $D^{*}$ allowed a looser requirement on the significance of separation between the two vertices. 
- Vertex detachment:

- Čerenkov identification:

- Vertex quality

- Vertex isolation

- Kinematic requirements

- Track quality

The primary background reduction tool is the requirement on the vertex detachment $\ell / \sigma_{\ell}$. It is often the only requirement necessary to expose a signal. In order to avoid region of low detection efficiency the available decay volume was limitted to the region upstream of the first trigger counter (TR1). This requirement caused a noticeable loss of events only in the $D^{ \pm}$case and will be discussed in the following section. The PASS1 linking algorithm allowed one microstrip track to connect to at most two PWC tracks. An electron and positron coming from a photon conversion in the target or microstrips usually appears as a single track in the SSD system which then gets resolved into two tracks in the PWC system after passing through the first analysis magnet. In order to reduce the contamination by these electrons/positrons, only tracks that were uniquely linked between the PWC and SSD systems were considered while forming charm decay candidates.

The processing of such a large data set inevitably caused double processing of certain events. To avoid double counting, all final data sets were passed through a "cleaning" algorithm that removed multiple copies of events.

\subsection{1 $\quad D^{ \pm} \rightarrow K^{\mp} \pi^{ \pm} \pi^{ \pm}$Decay}

The sample of $D^{ \pm}$candidates obtained through the algorithm discussed in the previous section was subjected to a final selection algorithm. These requirements included 

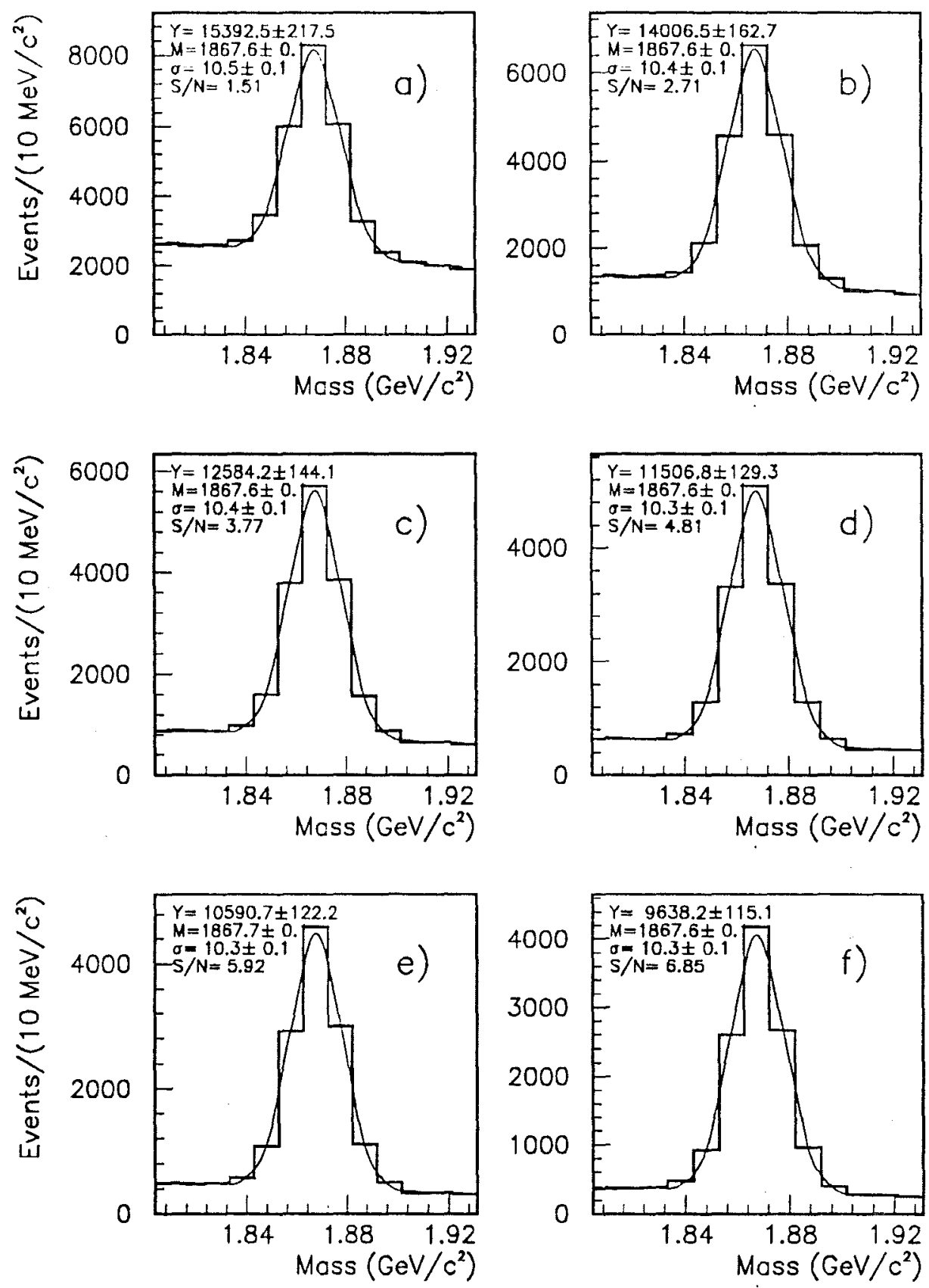

Figure 3.5. $K \pi \pi$ invariant mass distribution for various $\ell / \sigma_{\ell}$ requirements: a) $\ell / \sigma_{\ell}>5$; b) $\ell / \sigma_{\ell}>8$; c) $\ell / \sigma_{\ell}>11$; d) $\ell / \sigma_{\ell}>14$; e) $\ell / \sigma_{\ell}>17$; f) $\ell / \sigma_{\ell}>19$. 
Table 3.4. Cuts used to obtain the final $D^{ \pm} \rightarrow K^{\mp} \pi^{ \pm} \pi^{ \pm}$sample

\begin{tabular}{|c|c|}
\hline Requirement & Requirement Value \\
\hline $\begin{array}{c}\text { Linking between } \\
\text { the PWC and SSD }\end{array}$ & Singly Linked tracks only \\
\hline Kaon ID & ISTATP $=4,12$ \\
\hline Pion ID & ISTATP $\neq 1,4,8,12$ \\
\hline$\ell / \sigma_{\ell}$ & $>5,6, \ldots, 19$ \\
\hline $\begin{array}{c}\text { Confidence Level of } \\
\text { the Primary Vertex }\end{array}$ & $>1 \%$ \\
\hline $\begin{array}{c}\text { Confidence Level of } \\
\text { the Charm Vertex }\end{array}$ & $>1 \%$ \\
\hline Primary Vertex Location & $-5.5<$ PVZ $<-1.0 \mathrm{~cm}$ \\
\hline Charm Vertex Location & CVZ $<2.4 \mathrm{~cm}$ \\
\hline
\end{tabular}

tightening of the linking requirement, tightening of the Cerenkov identification of kaons and pions, improving the vertex quality, and imposing a cut on the location of the primary and secondary vertices. All the requirements imposed at this stage are included in Table 3.4. The cut on the location of the primary vertex was imposed in order to restrict the production of $D^{ \pm}$'s in the beryllium target only. Restriction on the location of the secondary vertex was imposed in order to avoid decays occurring in the first trigger counter (TR1) and beyond. This was done in order to avoid the region of low reconstruction efficiency that may not be modeled well in Monte Carlo. This cut removed $2 \%$ of the events from the sample. With this cut the stiffer momentum distribution will have more events cut at long lifetimes. Fig. 3.5 displays the $D^{ \pm}$ signal for various values of $\ell / \sigma_{\ell}$ significance of separation between the two vertices.

\subsection{2 $D^{0} \rightarrow K \pi$ and $K \pi \pi \pi$ Decays}

The $D^{0}$ meson was observed through its two most prominent decay modes $K \pi$ and $K \pi \pi \pi$. The $D^{0}$ mesons observed in our experiment are either produced directly in the photoproduction process or indirectly from the decay of the photoproduced $D^{* \pm}$. 
Table 3.5. Cuts used to obtain the final $K \pi$ TAG sample

\begin{tabular}{|c|c|}
\hline Requirement & Requirement Value \\
\hline $\begin{array}{c}\text { Linking between } \\
\text { the PWC and SSD }\end{array}$ & Singly Linked tracks only \\
\hline Kaon ID & ISTATP $=4,12$ \\
\hline Pion ID & ISTATP $\neq 1,4,8,12$ \\
\hline$\ell / \sigma_{\ell}$ & $>0,1,2, \ldots, 19$ \\
\hline $\begin{array}{c}\text { Confidence Level of } \\
\text { the Primary Vertex }\end{array}$ & $>1 \%$ \\
\hline $\begin{array}{c}\text { Confidence Level of } \\
\text { the Charm Vertex }\end{array}$ & $>1 \%$ \\
\hline Primary Vertex Location & $-5.5<\mathrm{PVZ}<-0.7 \mathrm{~cm}$ \\
\hline Charm Vertex Location & $\mathrm{CVZ}<2.4 \mathrm{~cm}$ \\
\hline$m\left(D^{*+}\right)-m\left(D^{0}\right)$ & $145.5 \pm 2 \mathrm{MeV} / c^{2}$ \\
\hline
\end{tabular}

This fact was exploited to obtain two statistically independent samples for each of the two decay modes, the so called "TAG" and "NOTAG" samples depending on whether the $D^{0}$ candidate originated from a $D^{* \pm}$ or not. These two samples were separated based on the value of the mass difference between the invariant masses of the $D^{* \pm}$ and $D^{0}$ candidates. This extra kinematic constraint proved to be a very powerful tool in suppressing non-charm background owing to the small $Q$ value of this decay process causing a rather narrow mass difference distribution and thus allowing a tight requirement to be imposed on it. This requirement alone was sufficient to expose a prominent signal, causing the signal of the TAG sample be much cleaner than the NOTAG sample for the same $\ell / \sigma_{\ell}$ requirement. Requirements imposed on the set of events produced by the $D$ skim algorithm to obtain the final samples are listed in Tables 3.5 and 3.6. In this manner we have created four statistically independent samples of the $D^{0}$ decays which will be used in the following chapter to measure the $D^{0}$ lifetime.

The $K \pi$ NOTAG sample has a substantial amount of background, with signal 

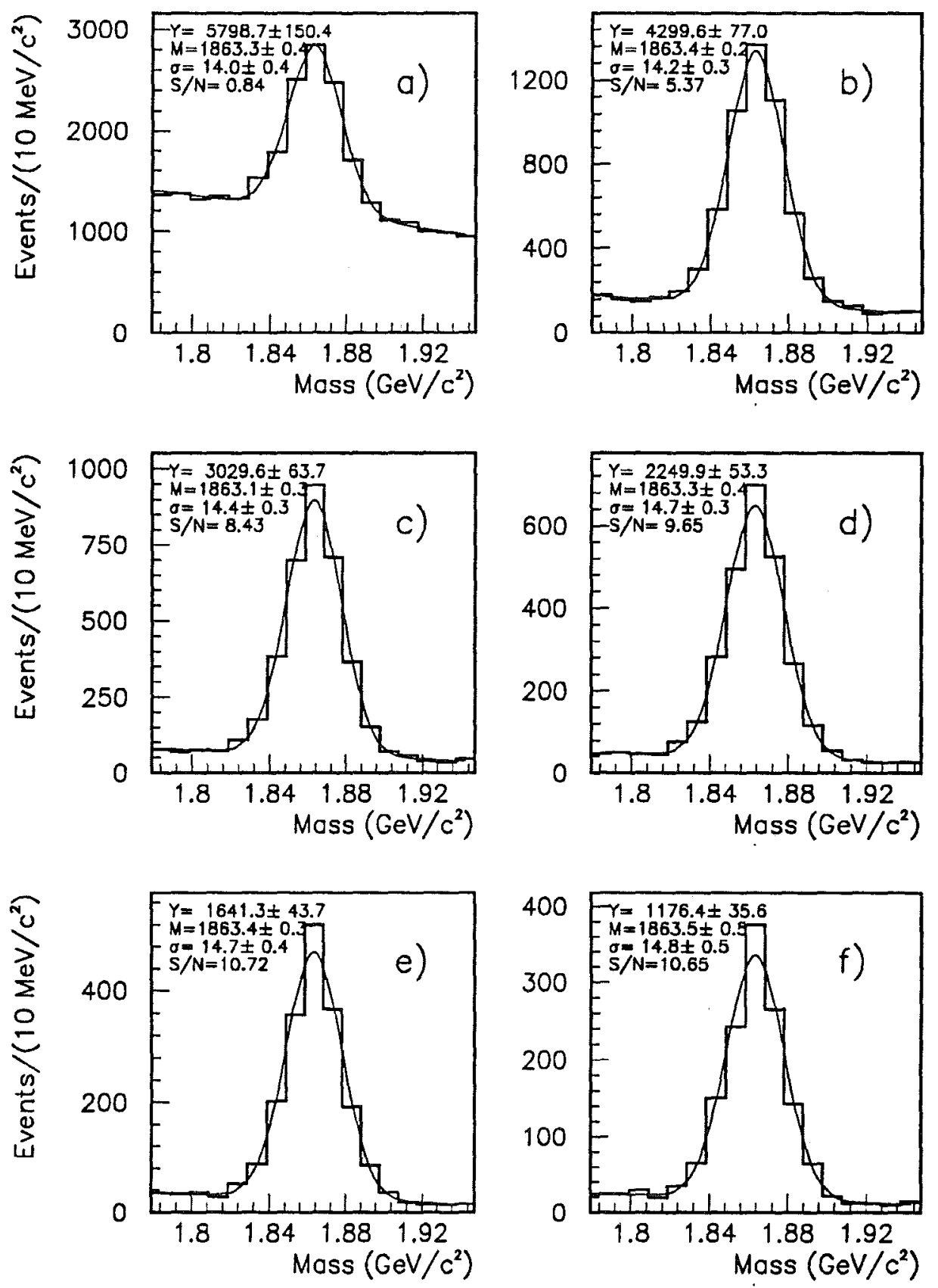

Figure 3.6. The invariant mass distribution of the $K \pi$ TAG sample for various $\ell / \sigma_{\ell}$ requirements: a) $\ell / \sigma_{\ell}>0$; b) $\ell / \sigma_{\ell}>3$; c) $\ell / \sigma_{\ell}>6$; d) $\ell / \sigma_{\ell}>9$; e) $\ell / \sigma_{\ell}>12$; f) $\ell / \sigma_{\ell}>15$. 
Table 3.6. Cuts used to obtain the final $D^{0} \rightarrow K \pi \pi \pi$ samples

\begin{tabular}{|c|c|}
\hline Requirement & Requirement Value \\
\hline \multicolumn{2}{|c|}{$\overline{D^{*+}} \rightarrow \pi^{+} D^{0}, D^{0} \rightarrow K^{-} \pi^{+} \pi^{+} \pi^{+}$} \\
\hline $\begin{array}{l}\text { Linking between } \\
\text { the PWC and SSD }\end{array}$ & Singly Linked tracks only \\
\hline Kaon ID & ISTATP $=4,12$ \\
\hline Pion ID & ISTATP $\neq 1,4,8,12$ \\
\hline$\ell / \sigma_{\ell}$ & $>2,3, \ldots, 19$ \\
\hline $\begin{array}{l}\text { Confidence Level of } \\
\text { the Primary Vertex }\end{array}$ & $>1 \%$ \\
\hline $\begin{array}{l}\text { Confidence Level of } \\
\text { the Charm Vertex }\end{array}$ & $>1 \%$ \\
\hline Primary Vertex Location & $-5.5<\mathrm{PVZ}<-0.7 \mathrm{~cm}$ \\
\hline Charm Vertex Location & $\mathrm{CVZ}<2.4 \mathrm{~cm}$ \\
\hline$m\left(D^{*+}\right)-m\left(D^{0}\right)$ & $145.5 \pm 2 \mathrm{MeV} / c^{2}$ \\
\hline \multicolumn{2}{|c|}{$\bar{D}^{0} \rightarrow K^{-} \pi^{+} \pi^{+} \pi^{+}$} \\
\hline $\begin{array}{l}\text { Linking between } \\
\text { the PWC and SSD }\end{array}$ & Singly Linked tracks only \\
\hline Kaon ID & ISTATP $=4,12$ \\
\hline Pion ID & ISTATP $\neq 1,4,8,12$ \\
\hline$\ell / \sigma_{\ell}$ & $>7,8, \ldots, 19$ \\
\hline $\begin{array}{l}\text { Confidence Level of } \\
\text { the Primary Vertex }\end{array}$ & $>1 \%$ \\
\hline $\begin{array}{l}\text { Confidence Level of } \\
\text { the Charm Vertex }\end{array}$ & $>1 \%$ \\
\hline Primary Vertex Location & $-5.5<\mathrm{PVZ}<-0.7 \mathrm{~cm}$ \\
\hline Charm Vertex Location & $\mathrm{CVZ}<2.4 \mathrm{~cm}$ \\
\hline$m\left(D^{*+}\right)-m\left(D^{0}\right)$ & $\neq 145.5 \pm 2 \mathrm{MeV} / c^{2}$ \\
\hline
\end{tabular}



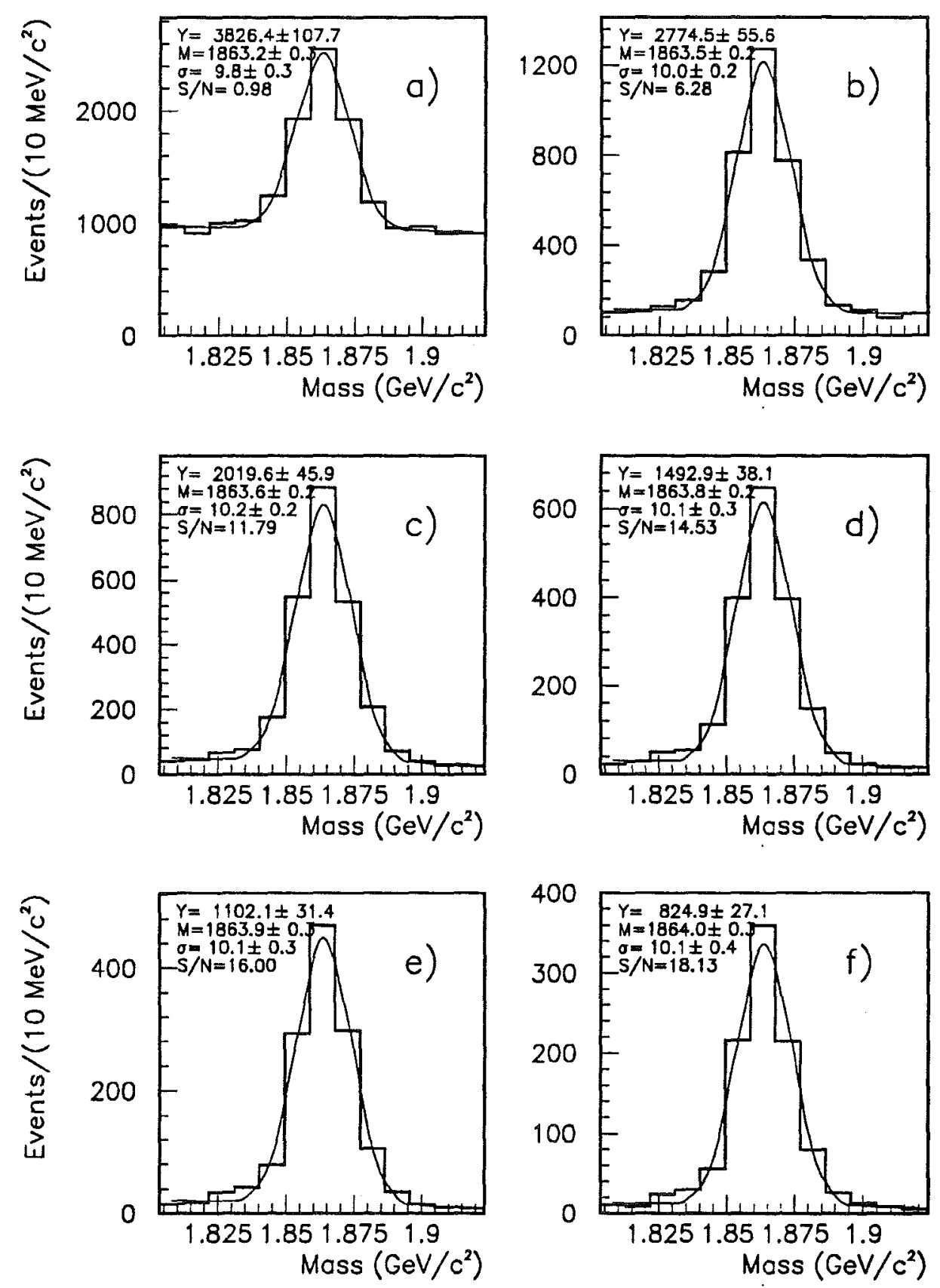

Figure 3.7. The invariant mass distribution of the $K \pi \pi \pi$ TAG sample for various $\ell / \sigma_{\ell}$ requirements: a) $\ell / \sigma_{\ell}>2$; b) $\ell / \sigma_{\ell}>5$; c) $\ell / \sigma_{\ell}>8$; d) $\ell / \sigma_{\ell}>11$; e) $\ell / \sigma_{\ell}>14$; f) $\ell / \sigma_{\ell}>17$. 

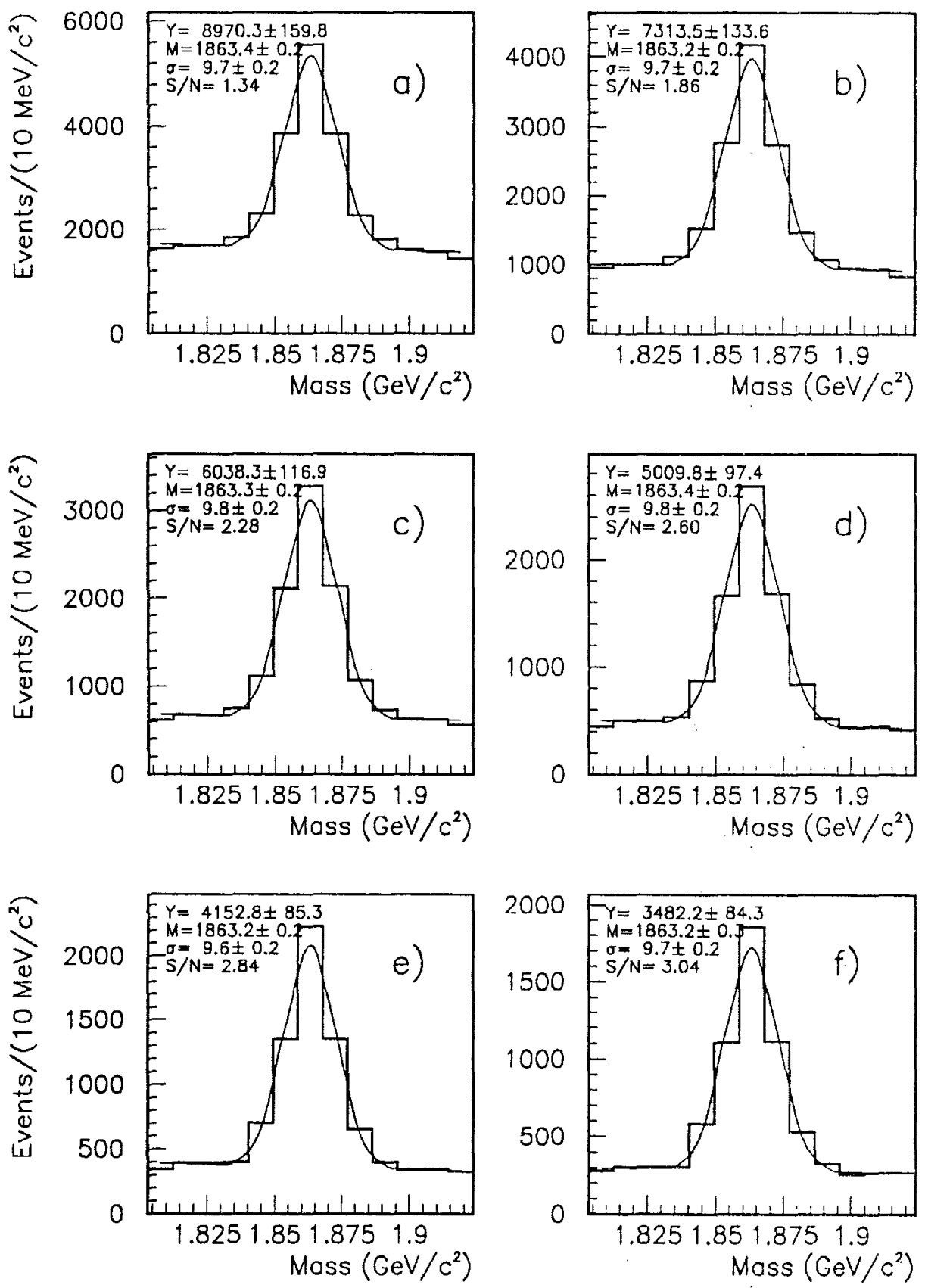

Figure 3.8. The invariant mass distribution of the $K \pi \pi \pi$ NOTAG sample for various $\ell / \sigma_{\ell}$ requirements: a) $\ell / \sigma_{\ell}>7$; b) $\ell / \sigma_{\ell}>9$; c) $\ell / \sigma_{\ell}>11$; d) $\ell / \sigma_{\ell}>13$; e) $\ell / \sigma_{\ell}>15$; f) $\ell / \sigma_{\ell}>17$. 
to noise ratio hardly exceeding one even for large values of $\ell / \sigma_{\ell}$. This fact together with rather different lifetimes of its mass sidebands intorduced a large systematic uncertanity in the lifetime causing us to abandone this sample in further studies. 


\subsection{3 $\quad D_{s} \rightarrow \phi^{0} \pi^{ \pm}$Decay}

In this analysis the $D_{s}^{ \pm}$meson was observed through its decay into $\phi^{0} \pi^{ \pm}$. This decay mode was obtained as a subset of the $\phi^{0}$ skim. Table 3.7 summarizes all the requirements imposed on the $\phi^{0}$ skim data set in order to obtain the final sample for the study of the $D_{s}^{ \pm}$lifetime. In addition to the standard vertex quality and separation cuts the existence of resonant structure was exploited to further improve the signal to noise ratio by cutting on the $\phi^{0}$ invariant mass. This cut turned out to be particularly powerful due to the narrow natural width ${ }^{8}$ of the $\phi^{0}$ resonance and a good (see Fig. 3.4) spectrometer invariant mass resolution of the $K^{+} K^{-}$system. This topology involves the decay of a pseudoscalar meson into a vector and a pseudoscalar. By conservation of angular momentum, the angle $\theta$ measured between one of the daughters of the vector and the outcoming pseudoscalar in the rest frame of the vector meson ( $\phi^{0}$ meson) will have a distribution proportional to $\cos ^{2} \theta$. By contrast the background has a flat distribution in $\cos \theta$ allowing us to efficiently cut on this variable (see Fig. 3.9). A cut of $|\cos \theta|>0.3$ retained $97 \%$ of the signal while eliminating $30 \%$ of the background.

The invariant mass distribution of this signal after all the above cuts for three values of $\ell / \sigma_{\ell}$ cut are shown in Fig. 3.10. In this plot the prominent $D_{s}^{ \pm}$mass peak centered around $1.968 \mathrm{GeV} / \mathrm{c}^{2}$ is accompanied by the Cabibbo supressed decay $D^{ \pm} \rightarrow \phi^{0} \pi^{ \pm}$centered around $1.869 \mathrm{GeV} / c^{2}$.

\footnotetext{
"The natural width of the $\phi^{0}$ has been measured[Pdg 92] to be $\Gamma_{\phi}=4.41 \pm 0.07 \mathrm{MeV}$.
} 
Table 3.7. Cuts used to obtain the final $D_{s}^{ \pm}$sample

\begin{tabular}{|c|c|}
\hline Requirement & Requirement Value \\
\hline Linking between & Singly Linked tracks only \\
the PWC and SSD & \\
Kaon ID & ISTATP $=4,12$ or 7 if $\mathrm{p}>61.5$ \\
Pion ID & ISTATP $\neq 1,4,8,12$ \\
$\mathrm{~m}\left(K^{+} K^{-}\right)$ & $1.01-1.03$ \\
$\ell / \sigma_{\ell}$ & $>3,4, \ldots, 19$ \\
Confidence Level of & $>1 \%$ \\
the Primary Vertex & $>1 \%$ \\
Confidence Level of & \\
the Charm Vertex & $>0.3$ \\
$|\cos \theta|$ & CVZ $<2.4 \mathrm{~cm}$ \\
Primary Vertex Location & $-5.5<\mathrm{PVZ}<-0.7 \mathrm{~cm}$ \\
Charm Vertex Location & .
\end{tabular}



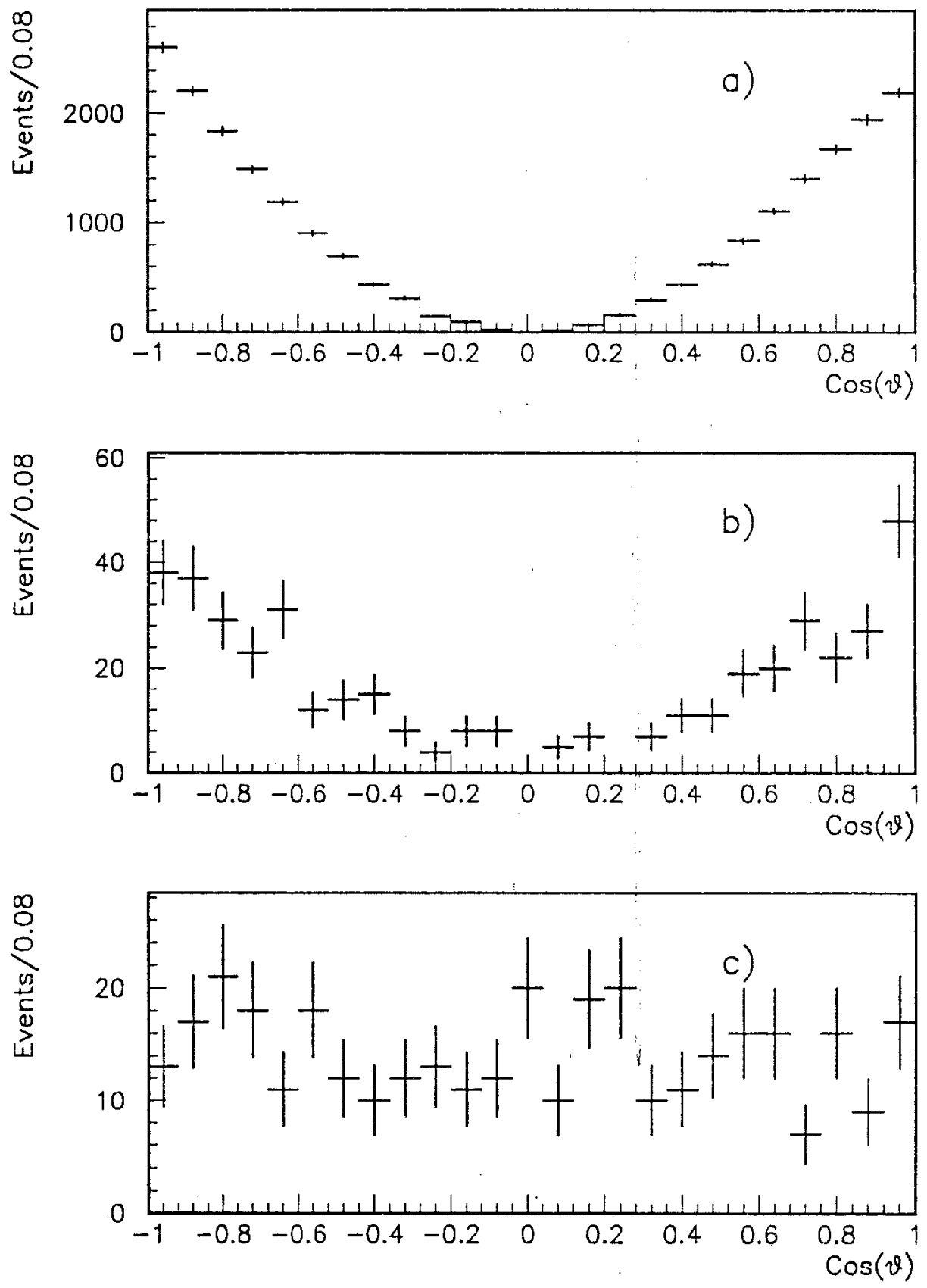

Figure 3.9. The $\cos \theta$ distributions for the $\phi^{0} \pi^{ \pm}$decay: a) Monte Carlo; b) $D_{s}^{ \pm}$signal region; c) mass regions outside the signal region. 

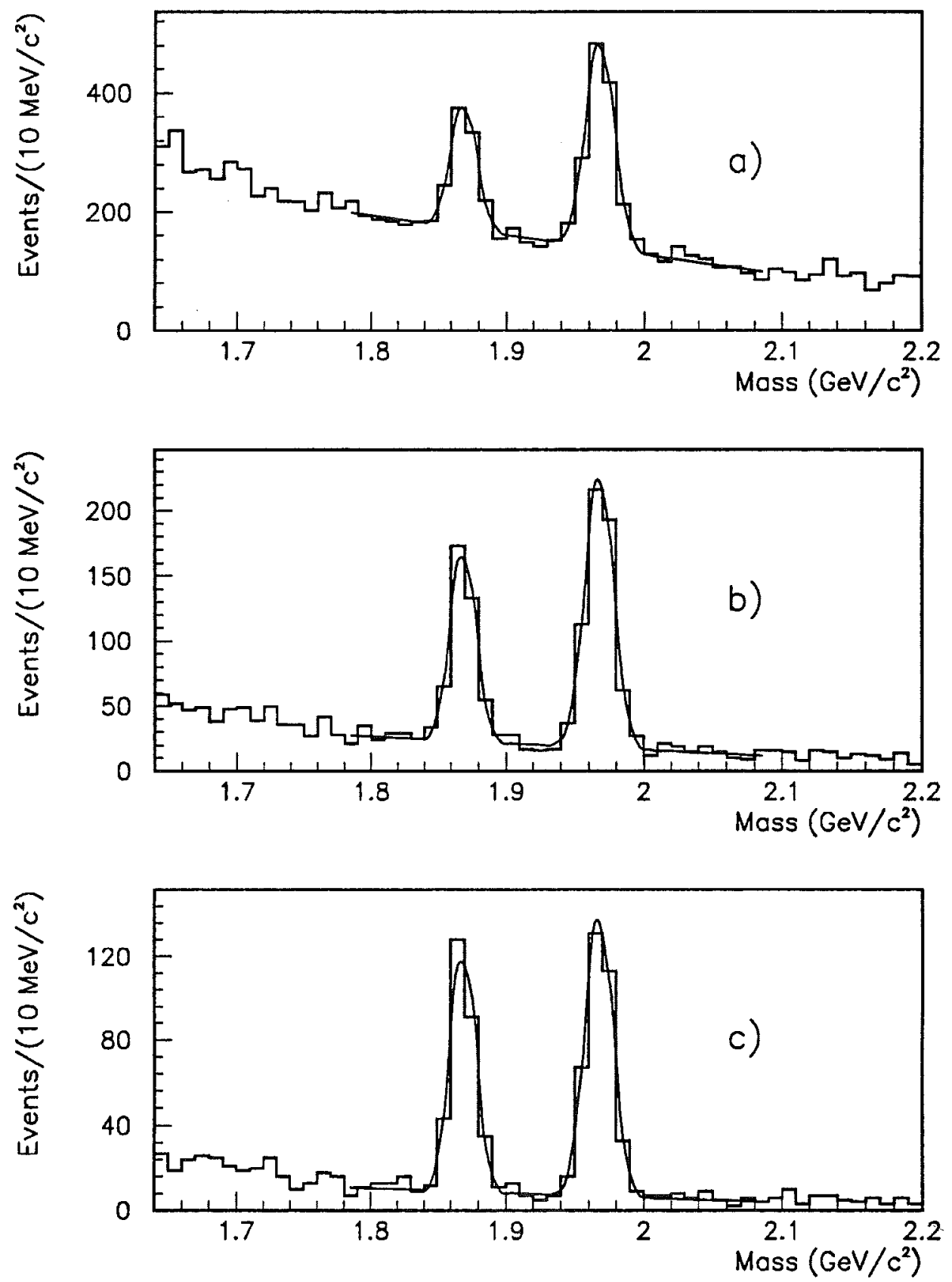

Figure 3.10. The invariant mass distribution of the $\phi^{0} \pi^{ \pm}$sample for various $\ell / \sigma_{\ell}$ requirements: a) $\ell / \sigma_{\ell}>3$; b) $\ell / \sigma_{\ell}>8$; c) $\ell / \sigma_{\ell}>13$. 


\section{CHAPTER 4}

\section{Charm Meson Lifetime Analysis}

This chapter describes in detail the measurement of the $D^{ \pm}, D^{0}$ and $D_{s}^{ \pm}$meson lifetimes. A binned maximum likelihood technique was used to fit the observed time distributions in order to extract the particular lifetime. The first section describes the employed method while the following sections describe the measurement of each of the three lifetimes and the evaluation of the corresponding systematic uncertainties.

\subsection{Lifetime Measurement Technique}

In the previous chapter "clean" $D^{ \pm}, D^{0}$ and $D_{s}^{ \pm}$signals were obtained from the data. The invariant mass distributions are shown in Fig. 3.5 through Fig. 3.10. The technique implemented to extract the lifetime utilized the binned maximum likelihood method to fit the observed time distributions. In particular a fit was performed to the histogram of the observed decay times for events that lay within the signal region of the invariant mass distribution $\left(m(D) \pm 2 \sigma_{m(D)}\right)$. All invariant mass distributions shown earlier contain signal as well as background events. Thus selection of events based on the value of the invariant mass necessarily involves both. So the maximum likelihood function constructed to fit the data time distribution has to properly model the signal as well as the background time evolution. The binned nature of this method allows the use of the time distribution of the invariant mass sidebands to represent the background under the real signal. 
In order to obtain the lifetime, two proper time histograms are created, one for events within the signal region and the other for events from the invariant mass sidebands. These histograms should span several mean lifetimes $\sim 6 \tau_{P D G}\left(\tau_{P D G}\right.$ is the particle data book [ $\mathrm{Pdg} 92]$ value of the lifetime). The maximum likelihood function is then constructed to fit the signal region time distribution utilizing the known functional form of the signal time evolution and the sideband time distribution to model the background. The fit is then performed to two parameters, the lifetime $(\tau)$ and the total number of background events under the signal $(B)$.

An alternative approach would have been to fit the observed lifetimes with a continuous maximum likelihood function [Cun 90a] on an event-by-event basis carefully modeling the background time evolution. This method was explored and was found impractical due to the large number of observed decays (in some cases maximum likelihood function would have consisted of over 50000 terms).

\subsubsection{The Likelihood Function}

The signal and sideband region time distributions were binned in a certain number of bins, typically $20-65$ spanning $6 \tau_{P D G}$ for a given signal. All of the observed decays occur in flight so the right variable to plot is the proper time given as $t=\ell / \gamma \beta c$, where $\ell$ is the distance the particle has traveled before decaying within the detector, and $\gamma \beta c$ is the laboratory frame Lorentz boost of the $D$ meson ${ }^{1}$. In order to avoid explicit requirement on the minimum proper time ${ }^{2}$, a so called reduced proper time $\left(t^{\prime}\right)$ is used instead. The reduced proper time is defined as $t^{\prime}=\left(\ell-N \sigma_{\ell}\right) / \gamma \beta c$, where $N$ represents the significance-of-detachment cut used. In the absence of absorption or acceptance effects the measured $t^{\prime}$ distribution for the $D$ events is expected to be

\footnotetext{
${ }^{1}$ Letter " $\mathrm{D}$ " will be used as a generic name representing any of the charm pseudoscalar mesons.

${ }^{2}$ This is necessary since the given $\ell / \sigma_{\ell}$ cut employed to isolate the signal contains an implicit cut on the lifetime.
} 


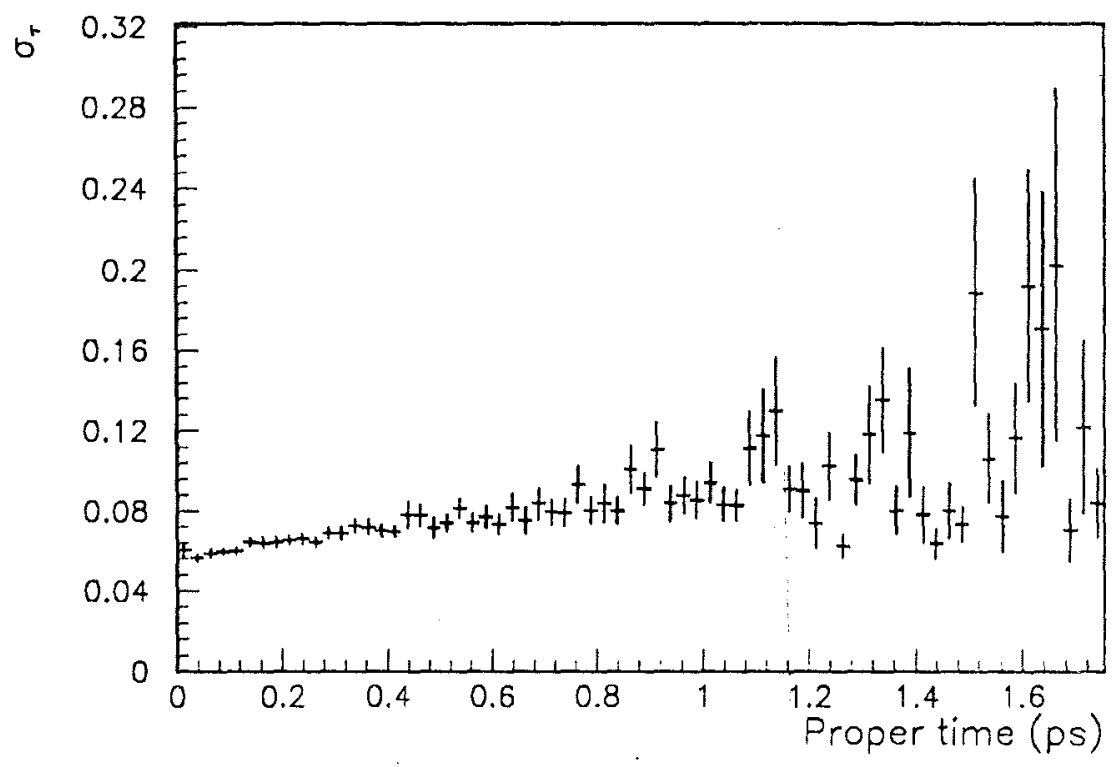

Figure 4.1. Error in proper time as a function of proper time

of the form $\exp \left(-t^{\prime} / \tau\right)$, where $\tau$ is the lifetime of the $D$. This is however true only as long as $\sigma_{\ell}$ is independent of $\ell$. (Studies of both data and Monte Carlo samples have shown that this implicit assumption is valid.) With this key assumption being true (see Fig. 4.1) this convenient formulation only shifts the origin of the proper time not effecting the shape or the slope of the exponential distribution.

The choice of the number of proper time bins is dictated by the available statistics and the experimental resolution in proper time. The experimental resolution was obtained by plotting the difference between the input and reconstructed proper times for Monte Carlo events. This distribution (see Fig. 4.2) is then fit with a Gaussian plus an exponential function. The width of the Gaussian distribution is $0.05 \mathrm{ps}$ representing the proper time resolution of our experiment for the states in question. On the other hand our sample sizes varied from 1000 to 20000 decays. In any event the fitted values were shown to be independent of the number of utilized bins.

The signal region is usually chosen to encompass as much of the signal as possible 


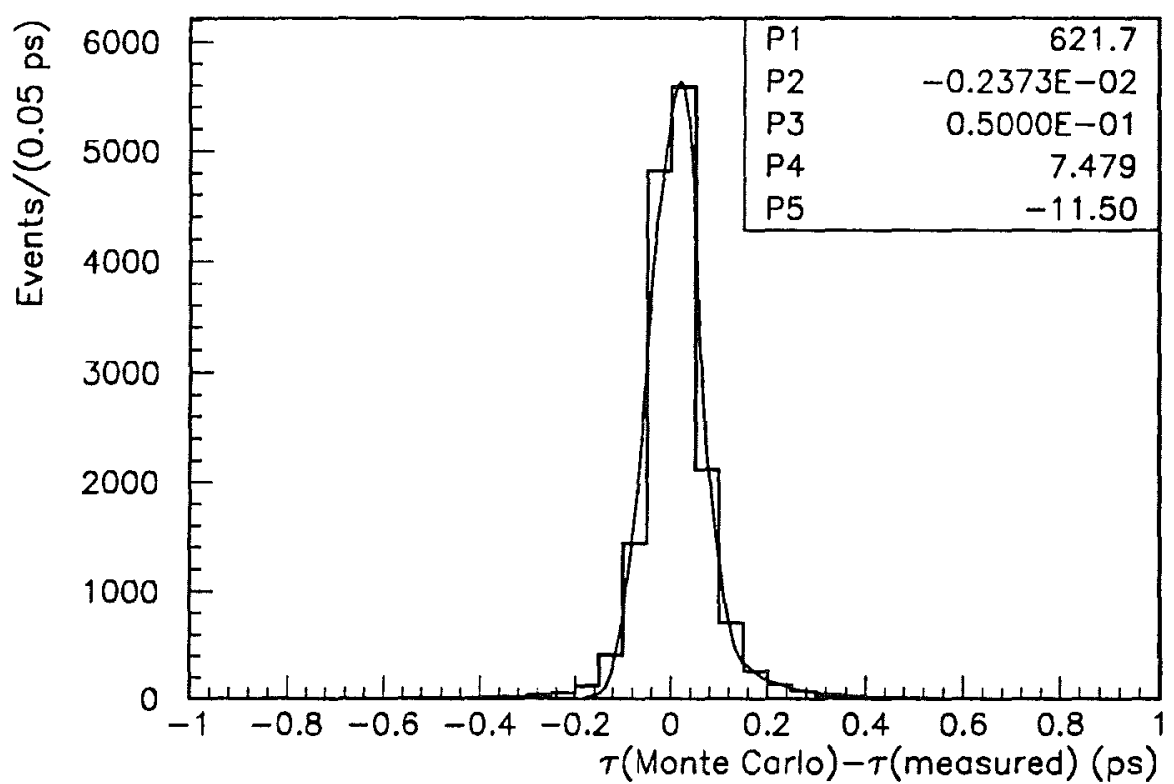

Figure 4.2. Distribution of the differences between input and reconstructed proper times for Monte Carlo. Overlayed on top is a result of a fit with a sum of a Gaussian and an exponential function.

yet retaining a good signal-to-noise ratio. We have chosen to define the signal region as the region $\pm 2 \sigma_{m(D)}$ around the mean $D$ mass $m(D)$ (thus utilizing $95 \%$ of the signal). Sideband mass regions should be representative of the background under the signal and as such should be chosen far enough from the signal region so that signal events are not included. On the other hand the time evolution of the background could be dependent on the invariant mass and thus a choice far enough away from the signal region may not be representative of the background under the signal. We have chosen to select two sideband regions one above and one below the signal as representative of the background. These two regions begin $\pm 4 \sigma_{m(D)}$ away from the mean $D$ mass and are $2 \sigma_{m(D)}$ wide. The actual widths of these regions and relative fractions of each used will be a source of the rather large systematic uncertainty of this method for states with low signal-to-noise ratio and will be extensively studied in the later sections. 
Having discussed the creation of the two fundamental histograms let us now construct the maximum likelihood function which is to fit the reduced proper time histogram in the signal region. Each reduced proper time bin contains true signal as well as background events, so the predicted number of events within the reduced proper time bin $i$ of the signal-histogram is given by

$$
n_{i}=N_{\mathrm{s}} \times P^{\mathrm{s}}\left(t_{i}\right)+N_{\mathrm{bg}} \times P^{\mathrm{bg}}\left(t_{i}\right)
$$

where $N_{\mathrm{s}}$ and $N_{\mathrm{bg}}$ are the total number of signal and background events in the signal region, while $P^{s}\left(t_{i}\right)$ and $P^{b g}\left(t_{i}\right)$ are the corresponding probabilities for events to have a decay time between $t_{i}$ and $t_{i}+\Delta t_{i}$. The $P^{s}\left(t_{i}\right)$ is proportional to the $\exp \left(-t_{i}^{\prime} / \tau\right)$ where $\tau$ is the lifetime of the signal. On the other hand $P^{b g}\left(t_{i}\right)$ is not known a priori and has to be modelled after the sideband data. In addition, the acceptance and resolution of the detector together with the hadronic absorption of charm particles and their daughters and chosen analysis cuts cause deviations from the pure exponential character of the signal by non-uniform selection of events with respect to their decay times.

By incorporating all the above facts, an operational definition of the predicted number of events in bin $i$ could be written in the following manner. First we set the nomenclature, by labeling the observed number of events in a reduced proper time bin $i$ (centered at $\left.t_{i}^{\prime}\right)$ in the signal and side band histograms as $s_{i}$ and $b_{i}$ respectively. The predicted number of events $n_{i}$ in a reduced proper time bin is then given by

$$
n_{i}=S \frac{f\left(t_{i}^{\prime}\right) \exp \left(-t_{i}^{\prime} / \tau\right)}{\sum f\left(t_{i}^{\prime}\right) \exp \left(-t_{i}^{\prime} / \tau\right)}+B \frac{b_{i}}{\sum b_{i}}
$$

where $S$ and $B$ are the total number of signal and background events in the signal 
region and $f\left(t_{i}^{\prime}\right)$ is the correction function. The fit parameters are $B$ and $\tau$ while $S$ is constrained to be the total number of signal region events minus $B$.

The function $f\left(t^{\prime}\right)$, derived from Monte Carlo simulation, corrects the proper time evolution of the signal for the effects of geometric acceptance, analysis cuts, hadronic absorption, and decay of charm secondaries.

Assuming a Poisson distribution of the entries in the signal-histogram the corresponding likelihood function, or the probability for obtaining the given histogram can be written as [Fro 79]

$$
\mathcal{L}_{\text {signal }}=\prod_{i=1}^{\text {bins }} \frac{n_{i}^{s_{i}}}{s_{i} !} \exp \left(-n_{i}\right)
$$

where $n_{i}$ is given by eq.4.2.

In order to relate $B$ to the number of background events expected from the population of the side bands while taking into account statistical fluctuations in the background level, a factor $\mathcal{L}_{\text {bg }}$ is included in the final likelihood function. The background level is thereby jointly determined from the invariant mass distribution and from the reduced proper time evolution in the side bands.

$$
\mathcal{L}_{\text {bg }}=\frac{\left(\mu_{\mathrm{bg}}\right)^{N_{\mathrm{bg}}}}{N_{\mathrm{bg}} !} \exp \left(-\mu_{\mathrm{bg}}\right)
$$

with $N_{\mathrm{bg}}=\sum b_{\mathrm{i}}$ and $\mu_{\mathrm{bg}}=B / R$ where $R$ is the ratio of widths of the signal and side band mass regions.

The final likelihood function is then given by the product of the two as

$$
\mathcal{L}=\mathcal{L}_{\text {signal }} \times \mathcal{L}_{\text {bg }}
$$


The two quantities utilized by the above model have statistical uncertainties associated with them. The first one is the uncertainty in the Monte Carlo calculation of $f\left(t_{i}^{\prime}\right)$ (caused by the finite Monte Carlo sample used for this purpose), and the second being the uncertainty associated with $b_{i}$ which are nothing but a normalized reduced proper time distribution of the sidebands. The model does not take into account these two sources of fluctuations, and thus will not be accounted for by the fit. In order to study the effect of these two uncertainties on the fitted lifetime value as well as its statistical error, several thousand Monte Carlo replicas of our final data sample were generated. The details of the modelling that went into these two studies will be discussed in the following section.

The above constructed likelihood function $\mathcal{L}$ or the joint conditional probability of observing $n_{1}, n_{2}, \ldots, n_{\ell}$ (where $\ell$ is the chosen number of bins) is a function of $\tau$ and $B$, whose best values are to be determined.

According to the Maximum-Likelihood Principle the best estimate of the unknown parameters are those values for which the likelihood function is maximized. So the goal is then to maximize the above likelihood function with respect to $\tau$ and $B$. Since $\mathcal{L}$ and the logarithm of $\mathcal{L}$ attain their maxima for the same value of parameters $\tau$ and $B$ we can maximize the $\log \mathcal{L}$ instead of $\mathcal{L}$ thus simplifying the functional form to be used from a product to a summation of terms.

In addition, maximizing a function is equivalent to minimizing the negative of a given function. Operationally this will allow the use of a general function minimizing package MINUIT [Jam 89]. So the function that is passed to MINUIT to be minimized is $-\log \mathcal{L}$, which then returns values of $\tau$ and $B$ corresponding to its minimum as the best estimates of these quantities. 


\subsection{Sources of Systematic Uncertainty}

In order to understand and examine all the possible sources of systematic uncertainties let us first examine in detail the production and the decay of a charm meson in the context of the E-687 detector.

According to the [Pdg 92] the mean proper path length (cr) of the $D^{+}, D^{0}$ and $D_{s}^{ \pm}$are $0.32,0.126$ and $0.135 \mathrm{~mm}$ respectively. In this experiment mesons are not produced at rest nor at any particular momentum value but with a spectrum ranging from 20 to $300 \mathrm{GeV} / \mathrm{c}$, with the mean value being $\approx 100 \mathrm{GeV} / \mathrm{c}$. Thus the average

mean path lengths of these three mesons in our detector are approximately 16, 6.3 and $6.8 \mathrm{~mm}$ respectively (assuming $\gamma \approx 50$ ).

The factors that directly effect the proper time distribution of the signal are:

- Transverse profile of the photon beam.

- Momentum of the charm mesons

- Hadronic absorption of charm mesons and their secondaries

- Decay of the secondaries

- Acceptance of the detector

- Analysis cuts

The correction function $f\left(t^{\prime}\right)$ is designed to account for all the above factors. In the cases where the $f\left(t^{\prime}\right)$ correction factor is large accurate modelling of the relevant processes is essential.

Factors that effect the measured lifetime in the fitting procedure are:

- Background parameterization

- Uncertainty in the $f\left(t^{\prime}\right)$ 
In the following subsections each of the above factors will be discussed in detail. For some systematic uncertainties an approach of estimating them was to find the derivative with respect to an uncertain parameter. Knowledge of the uncertainty of the given parameter then allows the propagation of the error in the lifetime due to this parameter.

\subsubsection{The $f\left(t^{\prime}\right)$ Correction Function}

The correction function $f\left(t^{\prime}\right)$ was derived from Monte Carlo in the following manner. A large Monte Carlo [Lin 90] [Gar 92] sample of the chosen decay was generated and analyzed in the same way that led to the observation of the final signal in data. The resulting reduced proper time distribution was then divided by the parent reduced proper time distribution to obtain the $f\left(t^{\prime}\right)$. Since the $f\left(t^{\prime}\right)$ factor used in our model isn't really a function but a finite array of values, each of the values has a statistical uncertainty associated with it directly related to the number of events observed in a given bin. These uncertainties are not included in the model so a study was performed to estimate the magnitude of this effect and thus determine the optimal size of the needed Monte Carlo sample.

To estimate the effect of the uncertainties of individual $f\left(t_{i}^{\prime}\right)$ values on the fitted lifetime, several thousand fits were performed on the given data sample with the $f\left(t_{i}^{\prime}\right)$ values randomly smeared at each iteration by a Gaussian of mean equal to the nominal $f\left(t_{i}^{\prime}\right)$ value and the width equal to its uncertainty. Fig. 4.3 displays the size of smearing introduced by the limited Monte Carlo statistics for each of the five decay modes. This distribution is fit with a Gaussian function whose width is used as an estimate of the uncertainty in the fitted lifetime caused by the finite Monte Carlo statistics. 

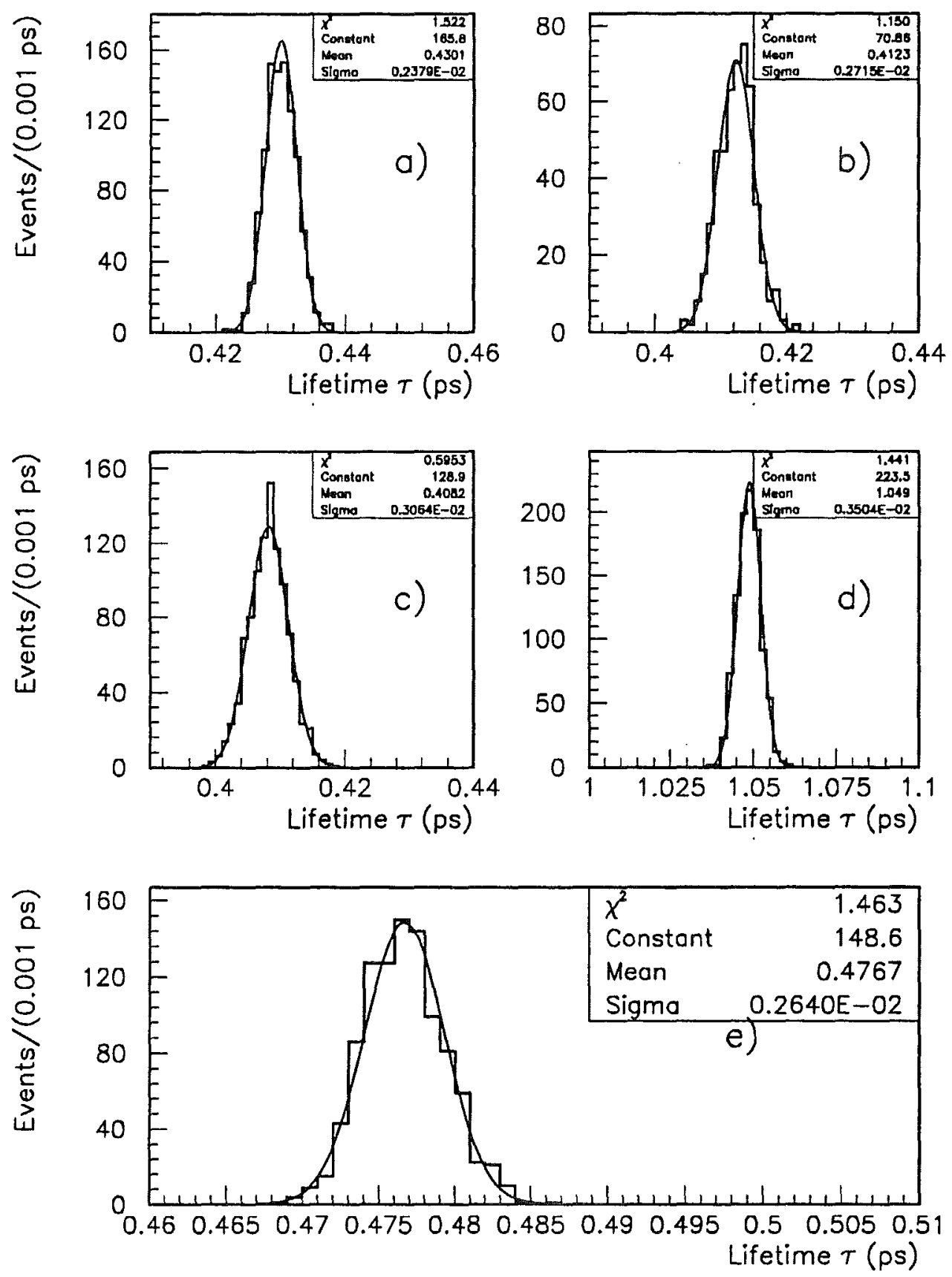

Figure 4.3. Uncertainty of the fitted lifetime caused by finite Monte Carlo statistic for $f\left(t^{\prime}\right)$ generated with: a) $400 \mathrm{~K} D^{0} \rightarrow K \pi$ TAG M.C. events; b) $400 \mathrm{~K} D^{0} \rightarrow K \pi \pi \pi$ TAG M.C. events; c) $400 \mathrm{~K} D^{0} \rightarrow K \pi \pi \pi$ NOTAG M.C. events; d) $1.75 \mathrm{M} D^{ \pm} \rightarrow K^{\mp} \pi^{ \pm} \pi^{ \pm}$M.C. events; e) $500 \mathrm{~K} D_{s}^{ \pm} \rightarrow \phi^{0} \pi^{ \pm}$M.C. events. 


\subsubsection{Uncertainty in the Photon Beam Profile}

The photons impinging on the Beryllium target have a given distribution in the transverse $x-y$ plane. This distribution is closely followed by the distribution of the $D$ production vertices. The acceptance of a given $D$ besides other factors is a function of the decay vertex coordinates $(x, y, z)$. The $D$ 's produced centrally (close to the $z$-axis) have on average a larger acceptance than those produced laterally (away from the $z$-axis) due to the finite acceptance of the SSD system. The $D$ 's decaying closer to the microstrips have on average higher acceptance than those decaying further away. Putting these two facts together one can easily imagine that observed time distributions for a decay point at the center of the target and at the point close to the edge would be different. The difference between the true and simulated beam profile (utilized to account for this effect) could result in a systematic bias. The photon beam profile along $\mathrm{x}$ and $\mathrm{y}$ directions is shown in Fig. 4.4 for the $D^{0}$ and $D^{ \pm}$samples. These figures show that the uncertainty in the fraction of the beam in a given bin is $\approx 5 \%$. In order to estimate the systematic uncertainty caused by this effect the Monte Carlo sample is divided into three transverse regions inner, middle and outer which are then fit utilizing the standard $f\left(t^{\prime}\right)$. Calculating the derivative of fitted lifetime with respect to the average radius and taking the above estimate for the uncertainty of the beam in the outer region a corresponding error in the fitted lifetime could be estimated.

\subsubsection{Uncertainty in the D Momentum Spectrum}

In the previous section we have pointed out that different z-positions with respect to the microstrip system have different acceptance. The higher momentum particles tend to decay closer to the miscrostrips than those of lower momentum, and hence have larger acceptance. This fact causes charm particles of radically different 

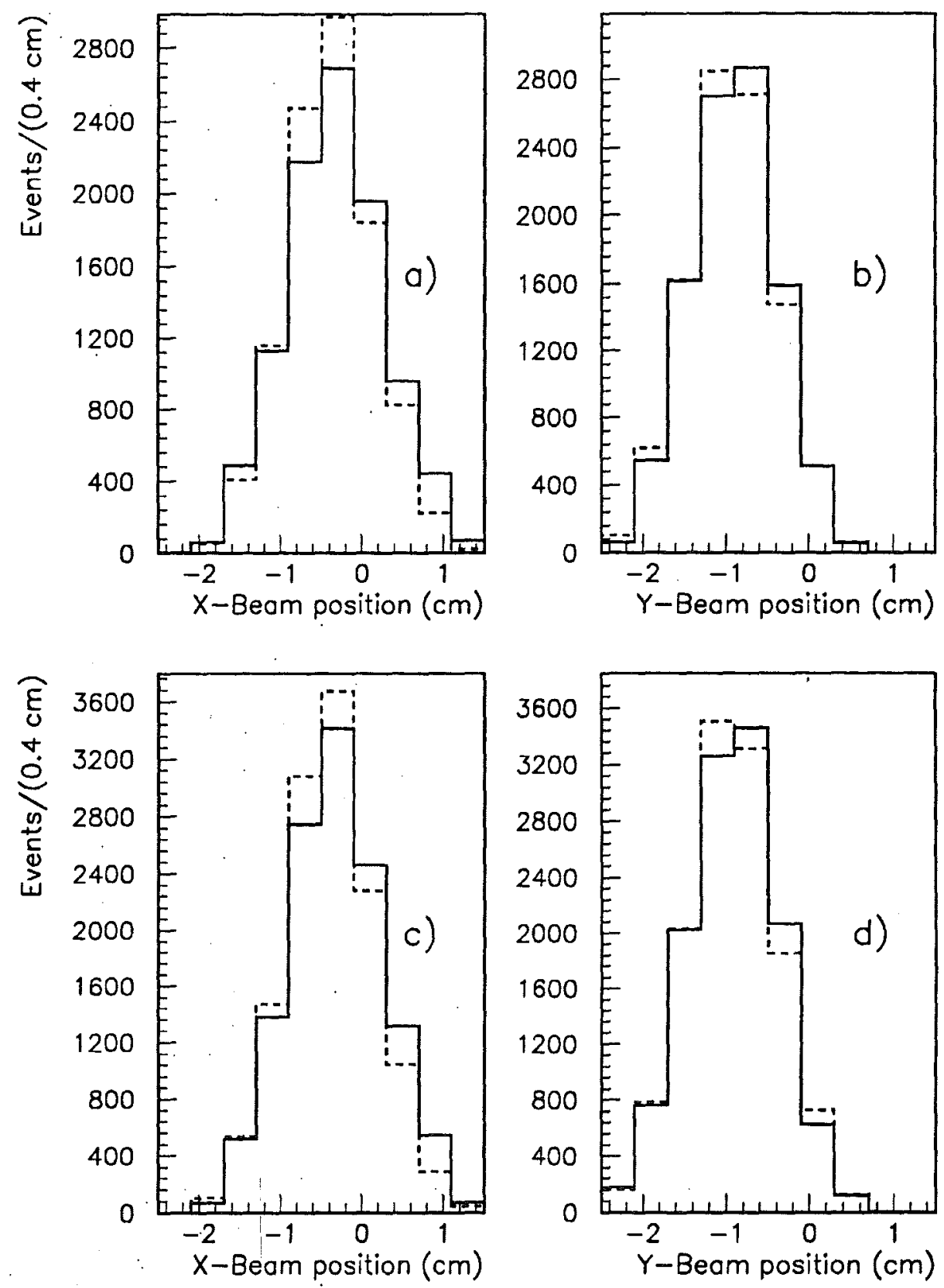

Figure 4.4. Transverse beam profile in the $\mathrm{x}$ direction in data and Monte Carlo for: a) $D^{0} \mathrm{X}$-profile; b) $D^{0} \mathrm{Y}$-profile; c) $D^{ \pm} \mathrm{X}$-profile; d) $D^{ \pm} \mathrm{Y}$-profile; solid and dashed lines represent data and Monte Carlo distributions respectively. 
momenta to have different observed decay-time distributions. Hence this difference between the observed and simulated momentum distributions, if not taken into account could result in a systematic bias. The systematic uncertainty caused by this effect is estimated in a similar manner by calculating the derivative of the fitted lifetime with respect to average momentum. That is if the lifetime changed by $\Delta \tau$ while the mean momentum changed by $\Delta P$ then the derivative is $\Delta \tau / \Delta P$. The uncertainty in average momentum has been estimated to be $\leq 2 \mathrm{GeV} / \mathrm{c}$, while the momentum regions are chosen in such a manner so that $\Delta P \approx 100 \mathrm{GeV} / \mathrm{c}$.

\subsubsection{Uncertainty in the Absorption Correction}

There are two effects that modify the observed decay time distribution, namely the hadronic absorption of the daughter particles and the absorption of charm particles themselves in the material that they pass through.

Hadronic absorption of the daughter particles if not taken into account would artificially increase the fitted lifetime since it causes depletion of events at short lifetimes (the daughters of short lived parents traverse more material than those which are long lived thus increasing their probability of absorption).

Conversely absorption of charm particles by the medium, if not taken into account, would tend to shorten the fitted lifetime since it causes a larger depletion of events at longer lifetimes.

Neither of these two effects were simulated in Monte Carlo and had to be included as post hoc corrections. The systematic uncertainty in the hadronic absorption of the secondaries arises because we do not know to what extent their elastic scattering causes severe mismeasurement of the parent particle. Uncertainty in the absorption of charm particles themselves comes from the fact that $D$ absorption cross section is unknown. In theory the $D$ absorption length is roughly a factor of two larger than the pion absorption length, but we use the latter as an upper bound. The pion cross 

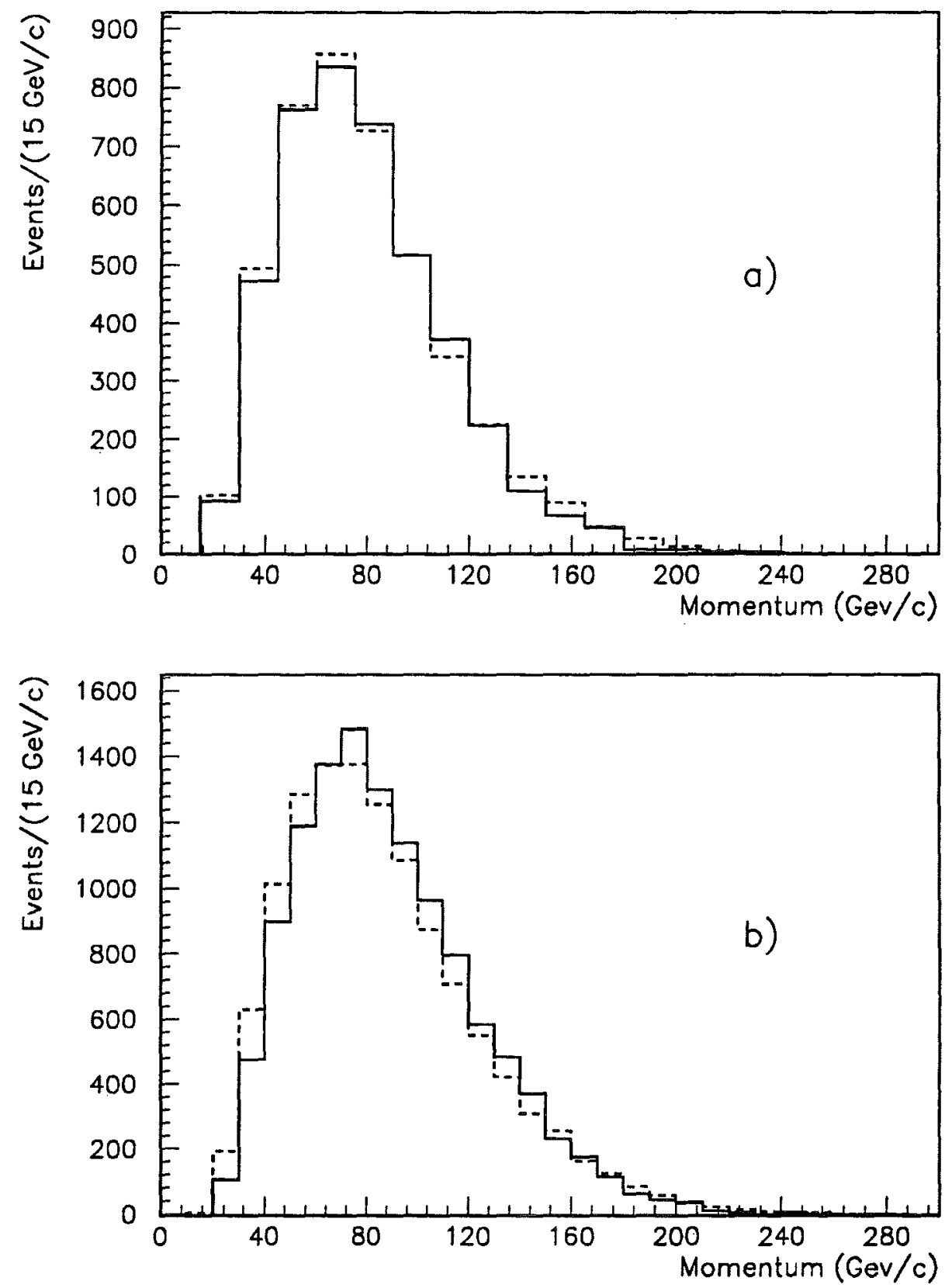

Figure 4.5. Comparison of $D$ momentum distributions in data and Monte Carlo for; a) $D^{0}$ sample; b) $D^{ \pm}$sample; solid and dashed histograms represent data and Monte Carlo distributions respectively. 

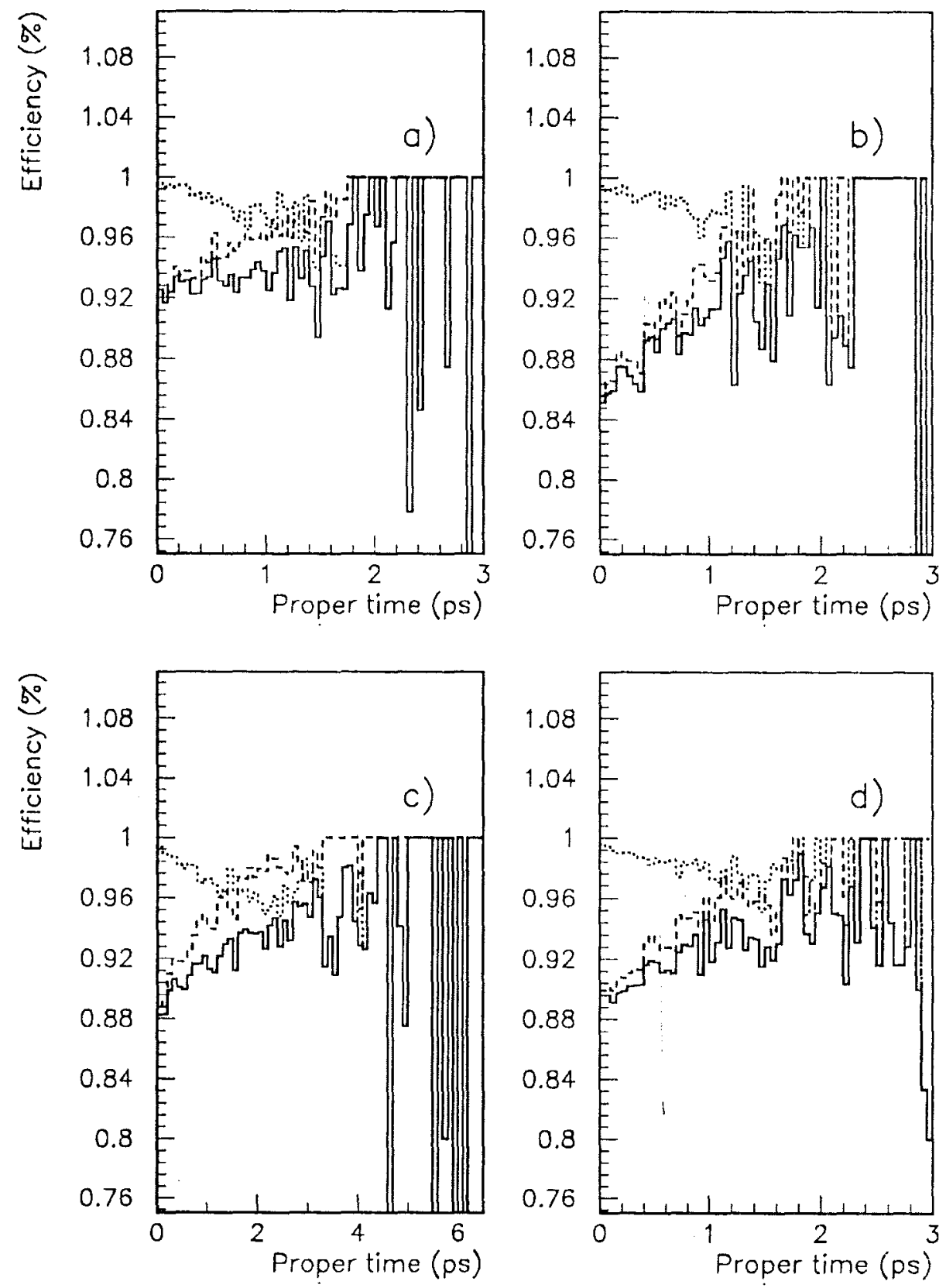

Figure 4.6. Effect of the hadronic absorption of charm particles and their secondaries as a function of proper time for; a) $D^{0} \rightarrow K \pi$; b) $D^{0} \rightarrow K \pi \pi \pi$; c) $D^{ \pm} \rightarrow K^{\mp} \pi^{ \pm} \pi^{ \pm}$; d) $D_{s}^{ \pm} \rightarrow \phi^{0} \pi^{ \pm}$. Dotted, dashed and solid lines represent effects of charm particle, daughter particle, and both absorptions together respectively. 
section [Den 73] was used to approximate the $D^{0}$ and $D^{+}$absorption cross sections, while the kaon cross section was utilized to simulate the $D_{s}^{ \pm}$absorption.

The estimates of these uncertainties were performed by varying the amount of absorption in Monte Carlo. The change of fitted lifetime caused by using the total nuclear cross section instead of inelastic cross section as a hadronic absorption of daughter particles was attributed as the systematic uncertainty due to the daughter absorption $\left(\sigma_{\text {elastic }} \approx 0.25 \sigma_{\text {total }}\right)$. The change of fitted lifetime due to the $50 \%$ decrease in the absorption of the parent particle was attributed to the uncertainty of charm particle absorption. These two effects tend to cancel each other so for the over all systematic uncertainity caused by the absorption we chose to quote the larger of the two effects.

\subsubsection{Uncertainty in the Background Modelling}

The likelihood function for the time evolution of the background under the signal is modeled by the time evolution of the chosen sidebands. This choice of background modelling introduces two concerns. First of all the fitting procedure does not take into account the statistical uncertainty of the sideband time distribution, and secondly the lifetime evolution of the background may depend strongly on the background mass.

In order to estimate the effect of fluctuations of the sideband time evolution several thousand Monte Carlo replicas of our final data sample were generated. These samples were generated to be identical in size to the final data sample. An exponential function was used to model the time evolution of the signal. Background time evolution was modelled by a sum of two exponential functions whose parameters were derived from data by fitting this functional form to the reduced proper time distribution of the utilized sidebands. These "mini experiments" were then fit for the lifetime $\tau$ and the total number of background events $B$ in the similar manner as the actual data. These studies allowed the evaluation of the errors returned by the fitter. For some of the 
sample this technique revealed the presence of a small statistical bias which was then taken into account while obtaining the final number.

The second concern proved to be more difficult to estimate because it was dependent on the final state in question and the multitude of possible contaminants that could cause the background time evolution to be mass dependent. The two most common sources of unwanted backgrounds are partially reconstructed charm decays (usually final states that involve a $\pi^{0}$ ), and the so-called reflection peaks caused by misidentification of one or more daughter particles from the charm decay. The value of the invariant mass of partially reconstructed states will tend to be lower than that of the completely reconstructed one. This will cause the upper and lower sidebands to have different lifetimes.

Reflection peaks can appear on either side of the peak depending on the misidentified particle in question. In particular we shall examine the reflection of $D^{ \pm} \rightarrow$ $K^{\mp} \pi^{ \pm} \pi^{ \pm}$into $D_{s}^{ \pm} \rightarrow \phi \pi^{ \pm}, D^{0} \rightarrow K^{-} K^{+}$into $D^{0} \rightarrow K^{-} \pi^{+}, \Lambda_{c} \rightarrow p K \pi$ into $D_{s}^{ \pm} \rightarrow \phi \pi$. The identified reflection peaks are easier to deal with since they can be removed by a cut on its actual mass.

The final results were obtained with equal parts of the low and high sidebands. To investigate the systematic uncertainties, the fraction of each sideband participating in the fit was varied between 0 and 1 . This effect is most dramatic in the cases where the ratio of signal to noise is low. For the systematic uncertainty we chose the avearge change in the fitted lifetime from the nominal value produced by utilizing $1 / 4$ and $3 / 4$ of the lower sideband.

\section{3 $D^{ \pm}$Lifetime}

The final $D^{ \pm}$sample consisted of $\approx 16000$ decays. The $f\left(t^{\prime}\right)$ correction function was derived from $1.75 \times 10^{6}$ Monte Carlo events. Due to its long lifetime the corrections applied by the $f\left(t^{\prime}\right)$ function are rather large. This is illustrated in Fig. 4.7 which 

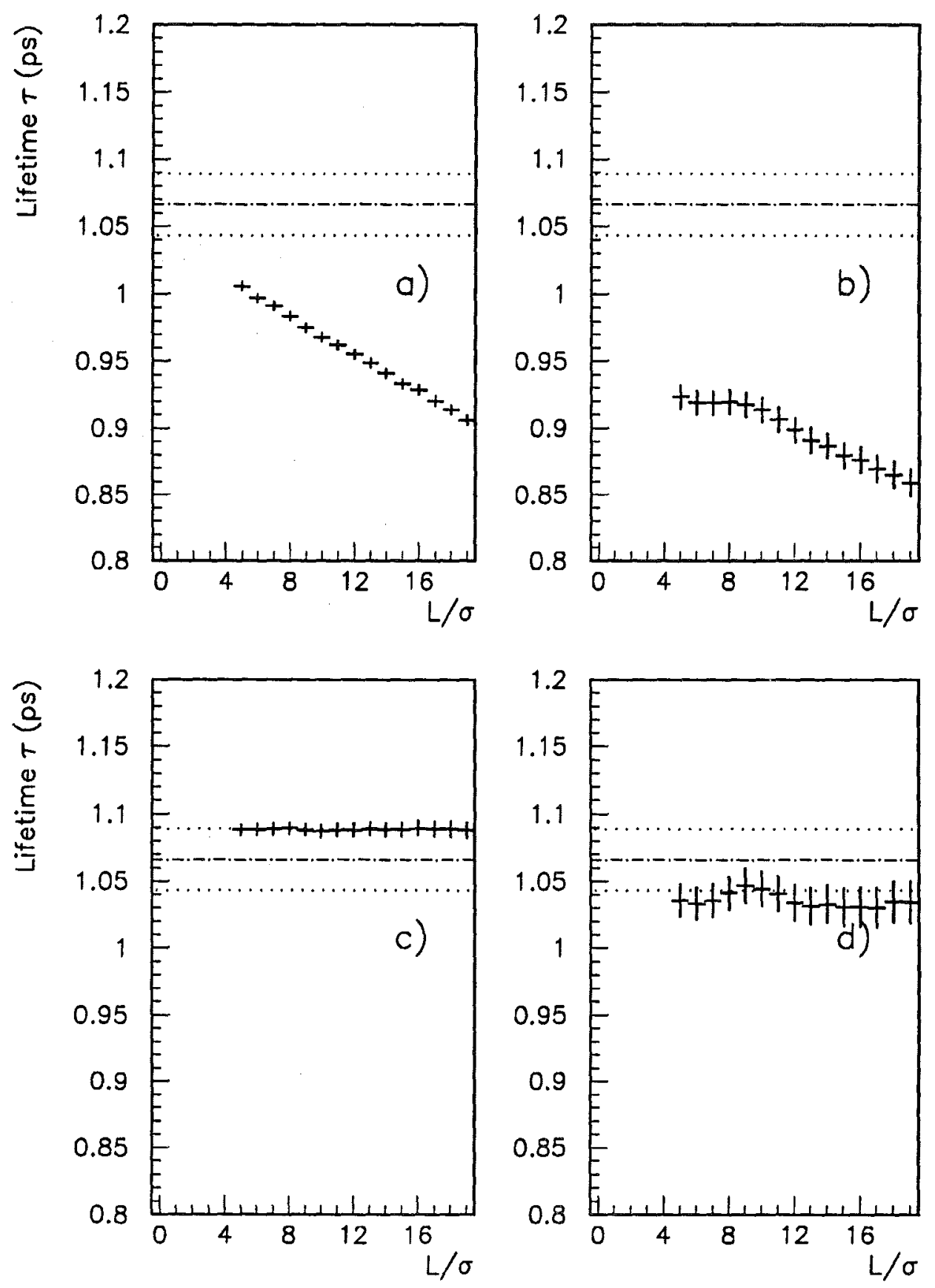

Figure 4.7. The fitted lifetime as a function of an $\ell / \sigma_{\ell}$ cut used for: a) Monte Carlo with $f\left(t^{\prime}\right)=1$; b) data with $f\left(t^{\prime}\right)=1$; c) Monte Carlo with the standard $f\left(t^{\prime}\right)$; d) data with the standard $f\left(t^{\prime}\right)$. Dash-dotted line represents the current world average value of the $D^{ \pm}$lifetime $(1.066 \pm 0.023 \mathrm{ps})$. The dotted lines represent the current error on the average value. 
compares the fitted lifetimes with and without the correction function $f\left(t^{\prime}\right)$ in data and Monte Carlo. The large $f\left(t^{\prime}\right)$ correction puts a strain on the Monte Carlo simulation. The uncertainties present in the Monte Carlo used to derive the $f\left(t^{\prime}\right)$ function could cause significant systematic uncertainties of the fitted lifetime. This plot clearly displays that we have reached a domain when the systematical uncertainties are becoming significant, requiring a careful study of the whole modelling process. In order to avoid possible systematic shift due to badly measured primary vertices, events with single track primaries were removed and the final fit was performed on the remaining sample.

The fitted lifetime displays a rather stable behavior with the $\ell / \sigma_{\ell}$ cut (see Fig. $4.7 \mathrm{~d}$ ). The choice of particular $\ell / \sigma_{\ell}$ cut at which to quote the lifetime was made based on the amount of systematic uncertainty caused by the sideband composition, while preserving the statistical significance. The final number is chosen to be quoted for $\ell / \sigma_{\ell}>15$. Fig. 4.8 shows the background-subtracted, $f\left(t^{\prime}\right)$ corrected, reduced proper time distribution for $\ell / \sigma_{\ell}>15$ requirement (solid histogram) together with the reduced proper time distributions of the signal (crosses) and sideband (dotted histogram) regions. An exponential function with its parameter equal to the fitted lifetime is overlayed. Fig. 4.9 shows the $f\left(t^{\prime}\right)$ as a function of reduced proper time for $\ell / \sigma_{\ell}>15$. The inefficiencies at larger lifetimes are caused by the requirement that the decay vertex occurs before the first trigger counter (TR1). This limits the decay volume which in turn causes the loss of events at longer lifetimes. The slowly rising slope at shorter lifetimes is caused by the hadronic absorption of the secondaries. The fitted lifetime is $1.042 \pm 0.015$ ps obtained for the choice of signal region between $1846.2-1888.6$ $\mathrm{MeV} / \mathrm{c}^{2}$ and two sideband regions $1803.8-1825.0$ and $1909.8-1931.0 \mathrm{MeV} / \mathrm{c}^{2}$.

To examine the effect of the fluctuations in the sideband reduced proper time distribution several thousand "mini-experiments" were performed as outlined in the previous section. Fig. 4.10a) shows the distribution of fitted lifetimes whose mean 


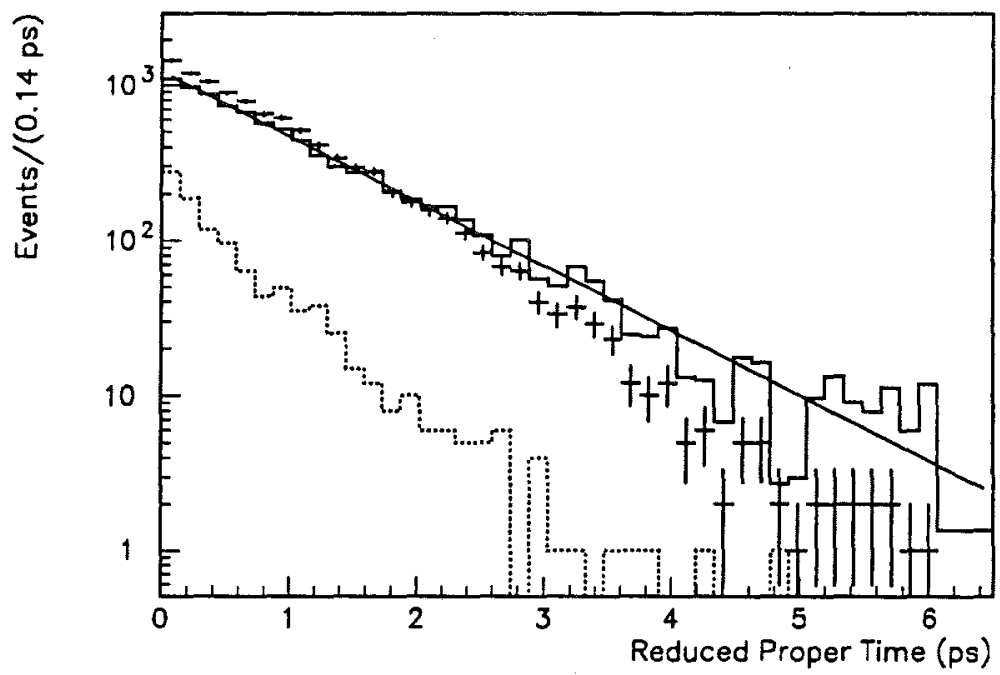

Figure 4.8. The background subtracted and Monte Carlo corrected lifetime evolution of the $D^{ \pm}$sample for $\ell / \sigma_{\ell}>15$ shown as solid line histogram. The crosses and dots represent the utilized reduced proper time distributions for the signal and sideband regions respectively. An exponential function with a fitted lifetime is overlayed.

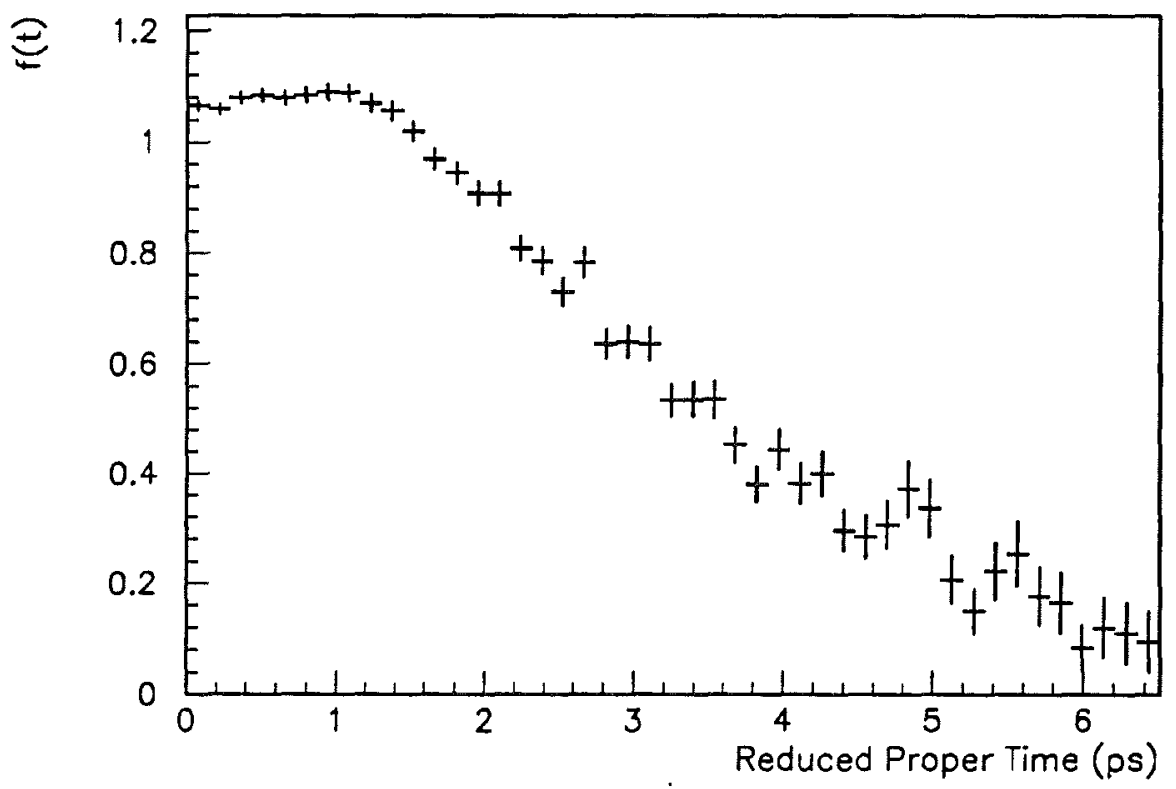

Figure 4.9. The correction factor $f\left(t^{\prime}\right)$ to the reduced proper time distribution of the $D^{ \pm}$decays as a function of reduced proper time. 

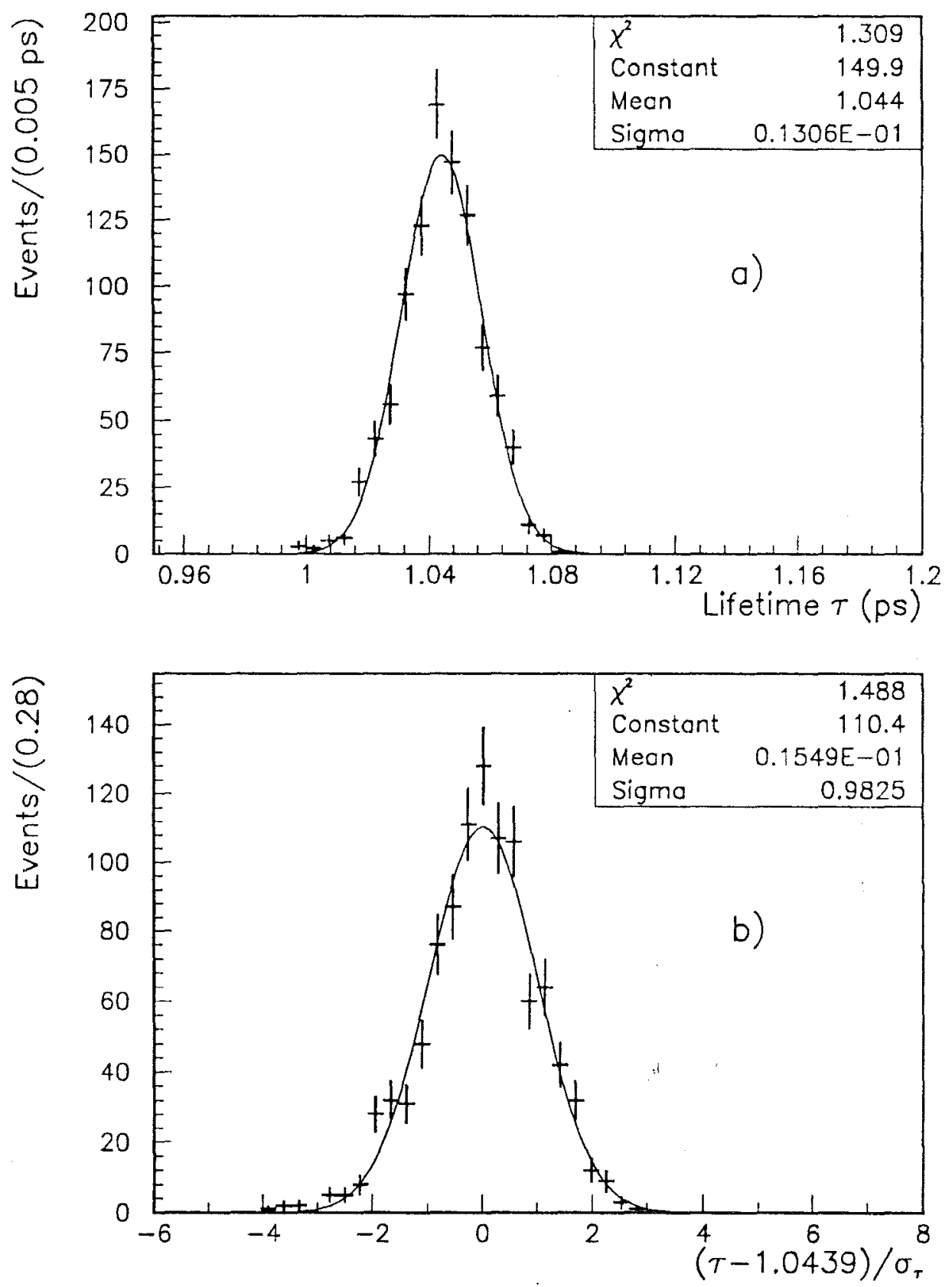

Figure 4.10. The result of simulated "mini-experiments": a) Distribution of fitted lifetimes; b) Distribution of fitted lifetimes minus the input lifetime divided by its error. 
Table 4.1. The $D^{ \pm}$Fit Parameters

\begin{tabular}{|c|c|}
\hline Parameter & Value \\
\hline Mean Mass & $1867.6 \pm 0.1 \mathrm{MeV} / c^{2}$ \\
Mass Resolution & $10.5 \pm 0.1 \mathrm{MeV} / c^{2}$ \\
Signal Mass Region & $1846.2-1888.6 \mathrm{MeV} / c^{2}$ \\
Low Sideband Mass Region & $1803.8-1825.0 \mathrm{MeV} / c^{2}$ \\
High Sideband Mass Region & $1909.8-1931.0 \mathrm{MeV} / c^{2}$ \\
$\ell / \sigma_{\ell}$ Requirement & $>15$ \\
Fitted Lifetime & $(1.042 \pm 0.015) \times 10^{-12} \mathrm{ps}$ \\
\hline
\end{tabular}

agrees well with the input lifetime value of the signal. A plot of the deviation of the fitted lifetimes from the input value divided by the error returned in the fit displays a Gaussian distribution (see Fig. 4.10b). The width of the Gaussian function fit to this distribution is $\approx 1$ showing that calculated errors are accurate.

The systematic uncertainties of the fitted lifetime caused by the $D^{ \pm}$momentum and photon beam profile uncertainties have been estimated by fitting three different regions in the Monte Carlo utilizing the standard $f\left(t^{\prime}\right)$. The corresponding fits are shown in Fig. 4.11 and Fig. 4.12 allowing us to determine the derivative of the fitted lifetime with respect to the mean momentum and radius respectively. From Fig. 4.5 and Fig. 4.4 we estimate the uncertainty in the mean momentum and radius to be 2 and $5 \%$ respectively.

The systematic uncertainty due to the particular choice of sidebands has been estimated by varying the fraction of the two sidebands that are utilized by the fit (see Fig. 4.13). The value of the systematic uncertanity caused by the sidebands is taken as the mean devation from the standard number caused by chosing $1 / 4$ and $3 / 4$ of the low sideband respectively.

All the systematic studies showed that the variation of the fitted lifetime from model uncertainties are of the same order or smaller than the statistical error of 

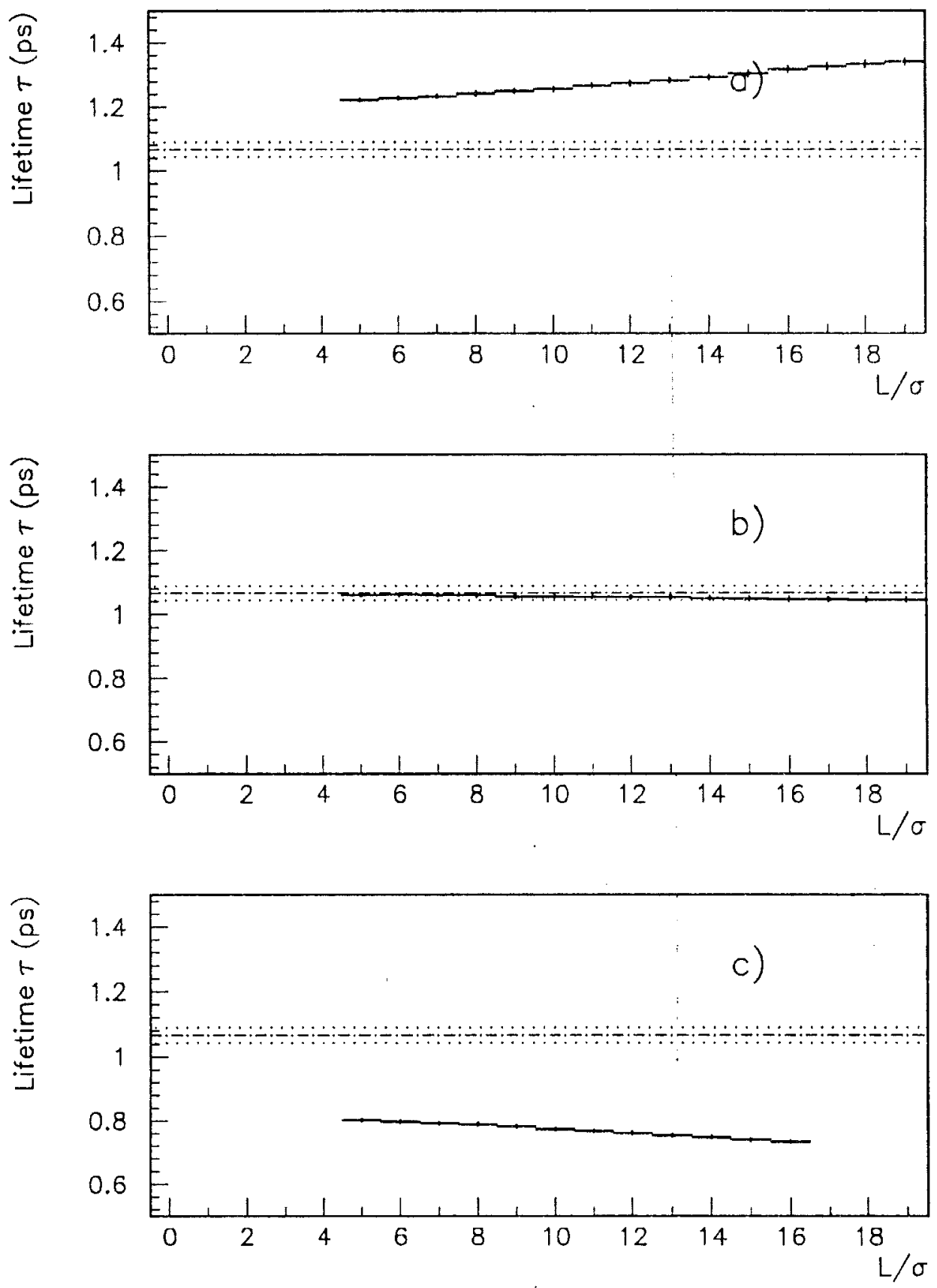

Figure 4.11. The $D^{ \pm}$fitted lifetime as a function of the $\ell / \sigma_{\ell}$ cut used for Monte Carlo utilizing the standard $f\left(t^{\prime}\right)$ for different momentum regions: a) momentum $P<80 \mathrm{GeV} / \mathrm{c}$; b) momentum $80<P<120 \mathrm{GeV} / \mathrm{c}$; c) momentum $P>120 \mathrm{GeV} / \mathrm{c}$. Dashed-dotted line represents the Monte Carlo input value for the $D^{ \pm}$lifetime. 

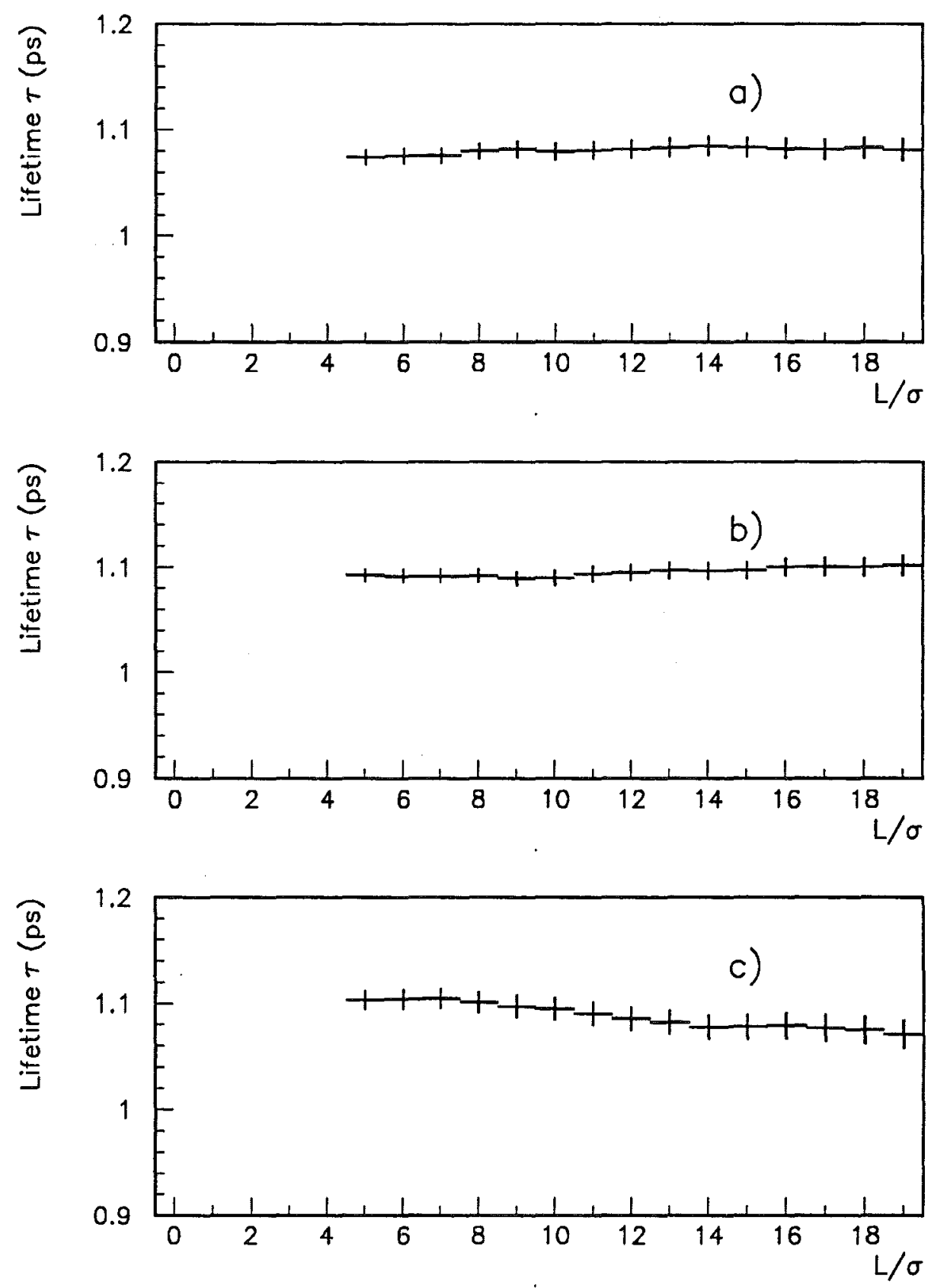

Figure 4.12. The $D^{ \pm}$fitted lifetime as a function of an $\ell / \sigma_{\ell}$ cut for Monte Carlo utilizing the standard $f\left(t^{\prime}\right)$ for different radial regions: a) inner Radius $R<0.5 \mathrm{~cm}$; b) middle Radius $0.5<R<1.0 \mathrm{~cm} ; \mathrm{c}$ ) outer Radius $R>1.0 \mathrm{~cm}$. 


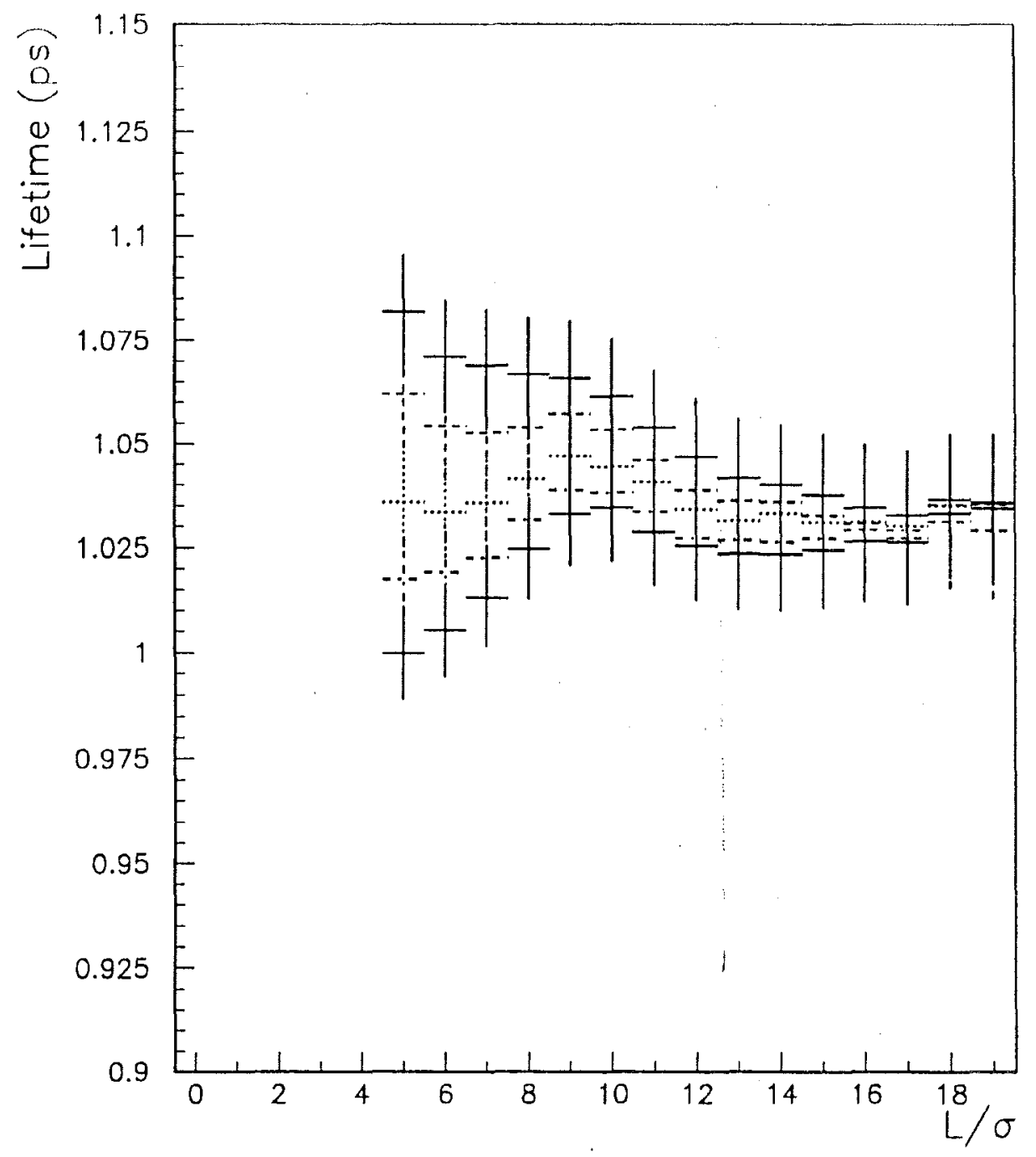

Figure 4.13. The $D^{ \pm}$fitted lifetime as a function of an $\ell / \sigma_{\ell}$ cut used for Monte Carlo utilizing the standard $f\left(t^{\prime}\right)$ for different fractions of the sideband regions: a) low side band $100 \%$ (solid line top most); b) low side band $75 \%$ (dashed line); c) low side band $50 \%$ (dotted line); d) low side band 25\% (dashed-dotted line); e) low side band $0 \%$ (solid line bottom most). 


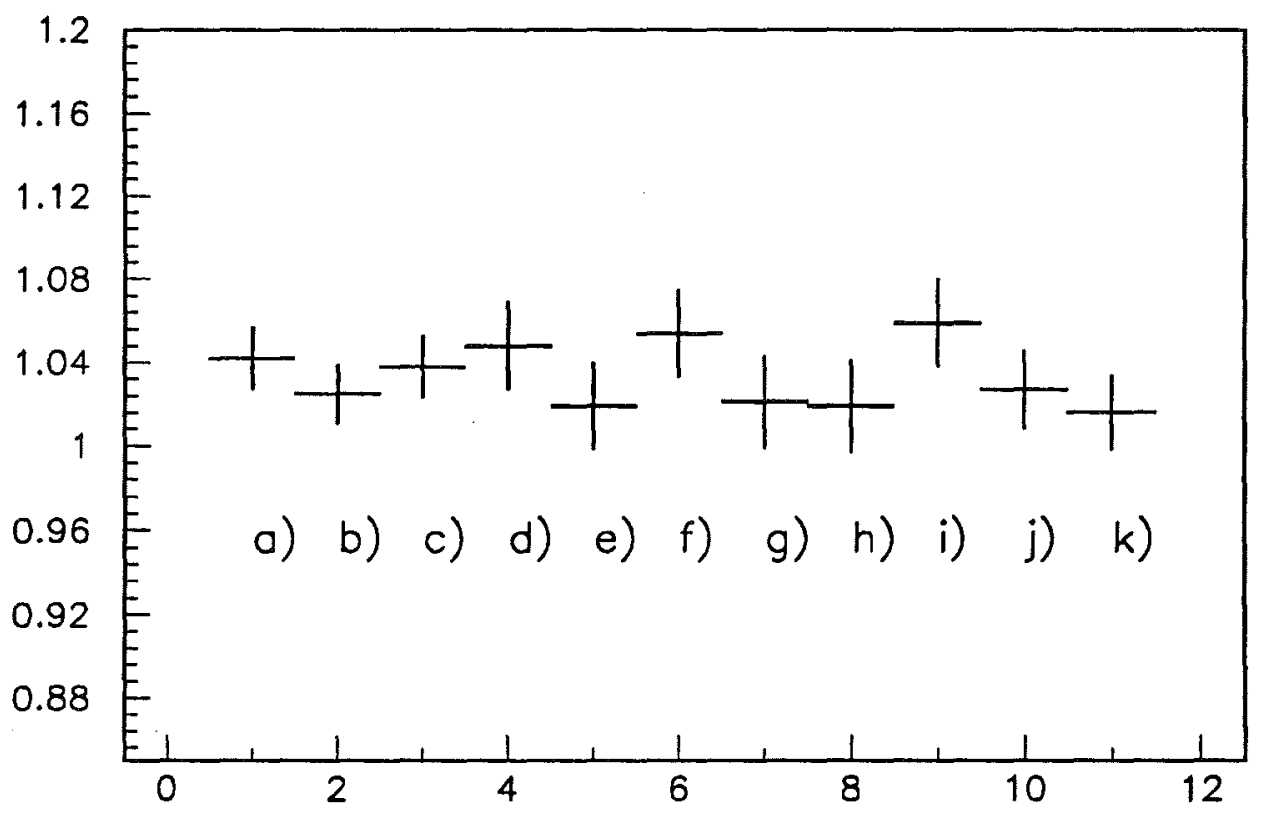

Figure 4.14. A comparison of measured $D^{ \pm}$lifetimes from different consistency checks and studies of systematic effects. The values are the results obtained: a) in this analysis; b) using $0 \%$ of low sideband; c) using $100 \%$ of low sideband; d) using upstream primary vertices only; e) using downstream primary vertices only; f) using $D^{+}$(particle) events only; g) using $D^{-}$(anti-particle) events only; $h$ ) using the 1990 data only; i) using the 1991 data only; j) using only decays that have occurred in the vacuum; $k$ ) PCL $>10 \%$, and DCL $>10 \%$ sample. 
Table 4.2. Systematic Uncertainties of the $D^{ \pm}$lifetime

\begin{tabular}{|c|c|}
\hline Source & Value $\left(\times 10^{-} 12\right)$ sec. \\
\hline$D^{-}$Momentum & $\mathbf{0 . 0 0 8 0}$ \\
Beam Profile & 0.0010 \\
Absorption & 0.0062 \\
Side Band & 0.0033 \\
$f\left(t^{\prime}\right)$ & 0.0035 \\
\hline Total & 0.0110 \\
\hline
\end{tabular}

this measurement. A comparison of measured lifetimes fọr the $D^{ \pm}$sample subject to several different constraints and partitions is shown in Fig. 4.14. Table 4.2 summarizes all the estimated systematic uncertainties. The overall systematic uncertainty has been estimated by adding all the individual systematic contributions in quadrature.

In summary we have measured the $D^{ \pm}$lifetime to be

$$
\tau\left(D^{ \pm}\right)=10.42 \pm 0.15 \pm 0.11 \text { ps }
$$

where the first error is statistical and second systematic.

\section{$4.4 D^{0}$ Lifetime}

In the previous chapter we have obtained the three statistically independent samples: $D^{0} \rightarrow K^{-} \pi^{+}$TAG, $D^{0} \rightarrow K^{-} \pi^{+} \pi^{-} \pi^{+}$TAG, and $D^{0} \rightarrow K^{-} \pi^{+} \pi^{-} \pi^{+}$NOTAG totalling to twenty thousand $D^{0}$ decays (see Fig. 3.6 through Fig. 3.8). The lifetimes of these three topologies were studied independently together with their corresponding systematic uncertainties. The $D^{0}$ lifetime is then obtained as a weighted average of these three measurements.

The utilized signal and sideband mass regions are listed in Table 4.3. The $f\left(t^{\prime}\right)$ correction functions were derived from $400 \mathrm{~K}$ Monte Carlo events generated for each 

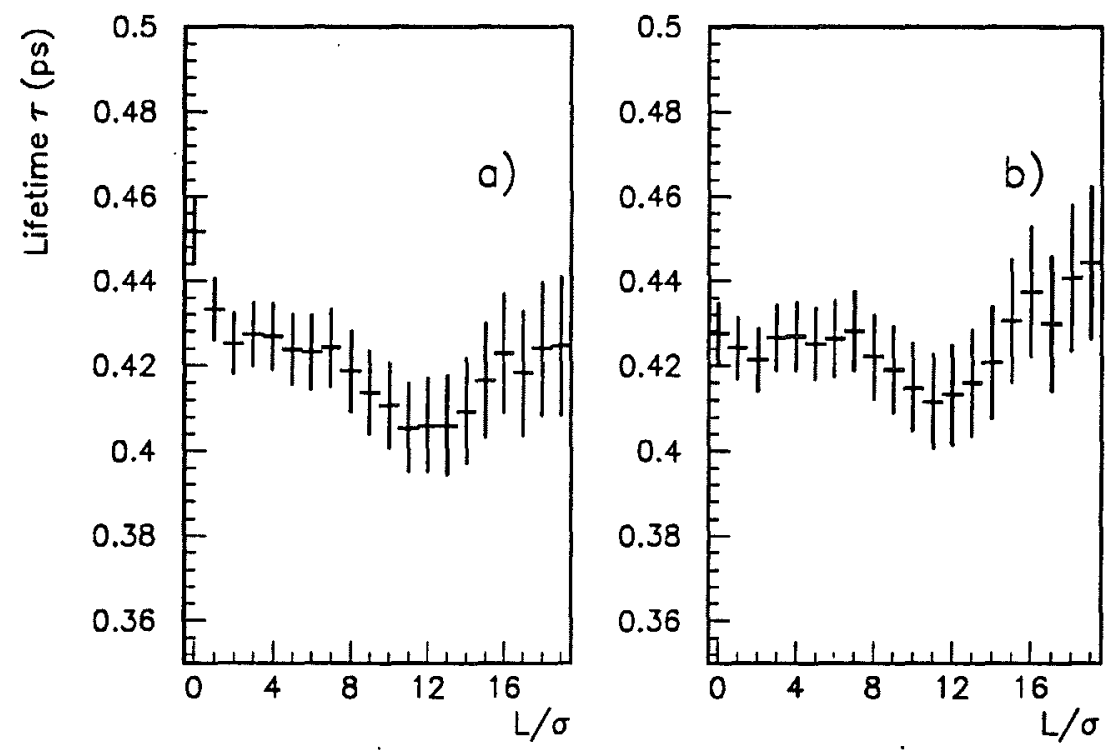

Figure 4.15. The fitted lifetime as a function of $\ell / \sigma_{\ell}$ for : a) $K \pi$ TAG sample with $f\left(t^{\prime}\right)=1$; b) $K \pi$ TAG sample with standard $f\left(t^{\prime}\right)$.

of the samples. Fig. 4.15 and Fig. 4.16 show the fitted lifetime as a function of $\ell / \sigma_{\ell}$ with and without the $f\left(t^{\prime}\right)$ correction. The correction introduced by the $f\left(t^{\prime}\right)$ is much smaller than in the case of the $D^{ \pm}$owing to the relative shortness of the $D^{0}$ lifetime. The fitted lifetimes do not significantly depend on the $\ell / \sigma_{\ell}$ cut so the value at which to quote the lifetime was chosen by minimizing the estimated uncertainty introduced by the choice of the sidebands, while maximizing the statistical significance. Fig. 4.17 through Fig. 4.19 show the variation of the fitted lifetime with

Table 4.3. Mass regions of the $D^{0}$ samples

\begin{tabular}{|c|c|c|c|}
\hline Sample & Signal Region & Low Sideband Region & High Sideband \\
\hline$K \pi$ TAG & $1.8353-1.8913$ & $1.7793-1.8073$ & $1.9193-1.9473$ \\
$K \pi \pi \pi$ TAG & $1.8433-1.8833$ & $1.8033-1.8233$ & $1.9033-1.9233$ \\
$K \pi \pi \pi$ NOTAG & $1.8433-1.8833$ & $1.8033-1.8233$ & $1.9033-1.9233$ \\
\hline
\end{tabular}



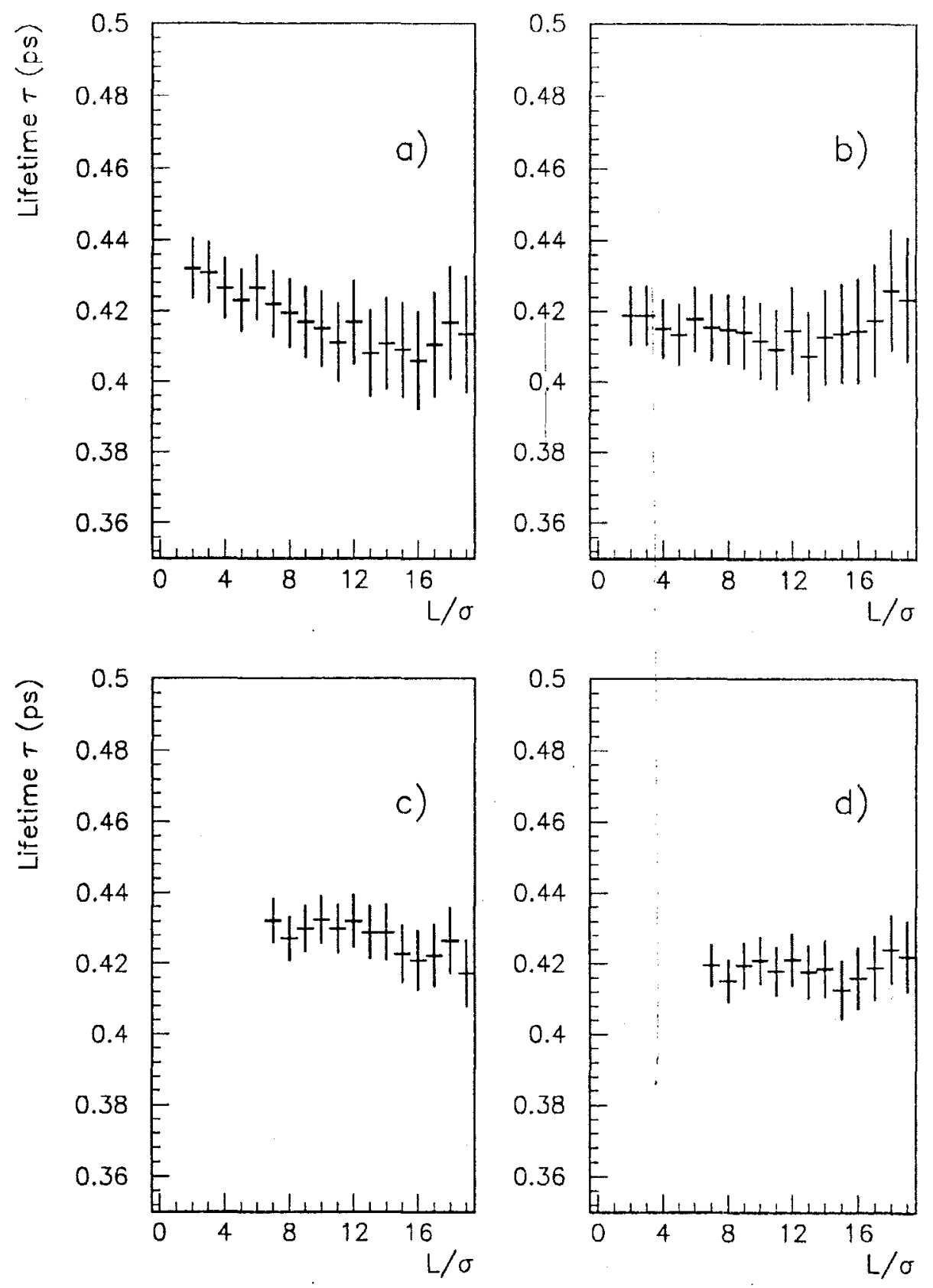

Figure 4.16. The fitted lifetime as a function of $\ell / \sigma_{\ell}$ for: a) $K \pi \pi \pi$ TAG sample with $f\left(t^{\prime}\right)=1$; b) $K \pi \pi \pi$ TAG sample with standard $f\left(t^{\prime}\right)$; c) $K \pi \pi \pi$ NOTAG sample with $f\left(t^{\prime}\right)=1$; d) $K \pi \pi \pi$ NOTAG sample with standard $f\left(t^{\prime}\right)$; 
$\ell / \sigma_{\ell}$ for various combinations of the sidebands for each of the three samples. The final fits to these three samples for the chosen $\ell / \sigma_{\ell}$ values are shown in Fig. 4.20 through Fig. 4.22.

To examine the effect of the fluctuations in the sideband reduced proper time distribution several thousand "mini-experiments" were performed for each of the three samples with background modelled after the individual sideband data distributions. The distribution of the fitted lifetimes are shown in Fig. 4.23a) and Fig. 4.24a,c). The mean of these three distribution should be equal to the input lifetime value. The difference between the mean and the input lifetime suggests the presence of the statistical bias of the fit. All three lifetimes have been corrected for the observed statistical bias (if any). The devation of the fitted lifetime from the input value divided by its error shown in Fig. 4.23b) and Fig. 4.24b,d) has a Gaussian distribution. The widths of the Gaussian functions fit to these distributions should be close to 1 if the errors estimation is accurate. Only in the case of the $K \pi \pi \pi$ NOTAG sample the Gaussian width is significantly larger then one indicating that the error returned by the fitter is underestimating the true error. In order to include the effect of fluctuations caused by the use of sidebands we have increased the statistical error of this sample by the corresponding amount.

The systematic uncertainties due to $D^{0}$ momentum, beam profile, absorption, and sideband choice were estimated in a similar manner as for the $D^{ \pm}$measurement. Table 4.4 summarizes the estimated uncertainties from these sources. The last row in this table is obtained by adding the contributions from the various sources in quadrature. Table 4.5 shows the measured lifetimes of these three samples together with corresponding statistical and systematic errors.

The lifetime of the $D^{0}$ is now estimated as the weighted average of the three 


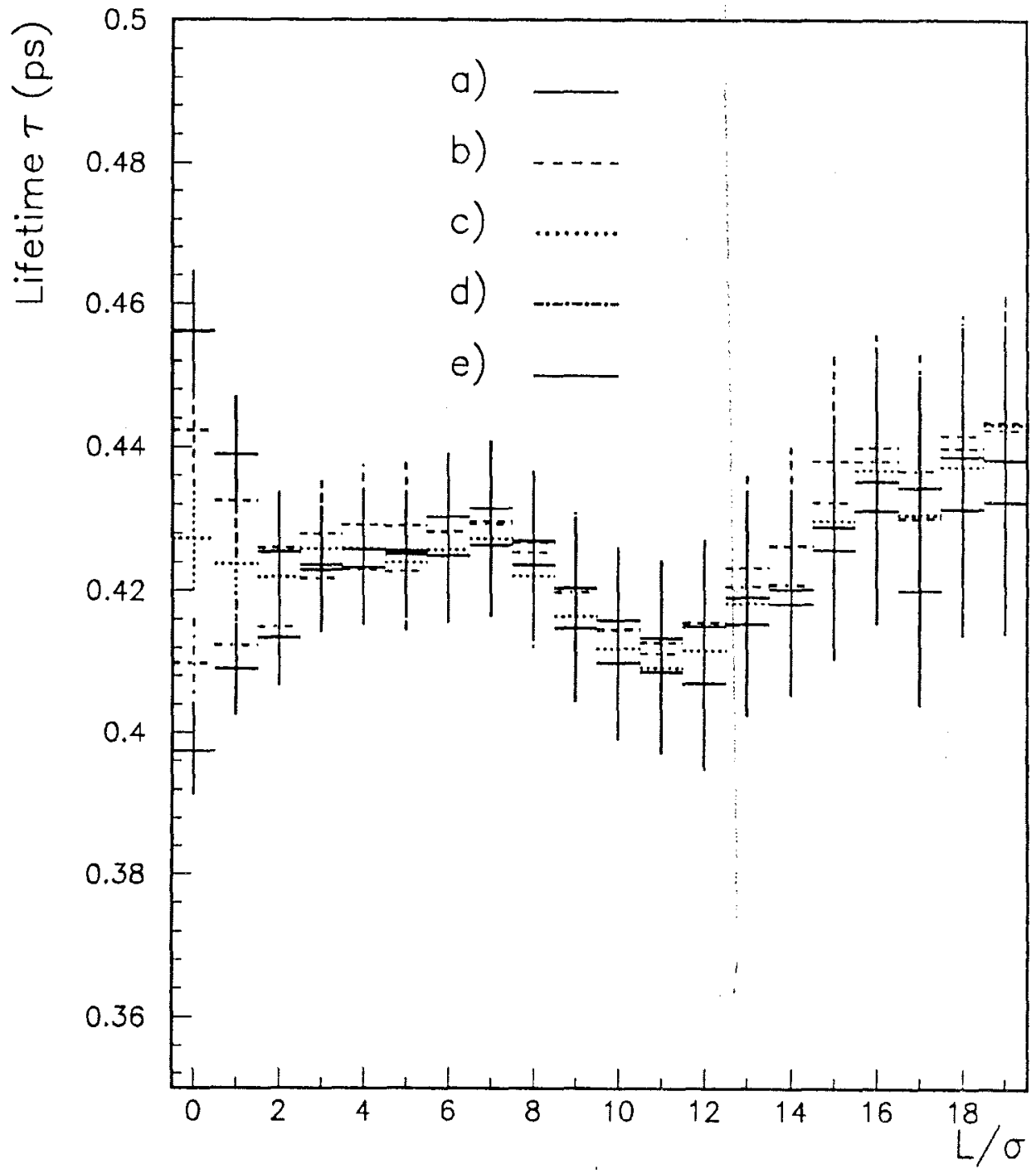

Figure 4.17. The $K \pi$ TAG sample lifetime as a function of $\ell / \sigma_{\ell}$ cut for the different fractions of sideband regions: a) low side band $100 \%$; b) low side band $75 \%$; c) low side band $50 \%$; d) low side band $25 \%$; e) low side band $0 \%$. 


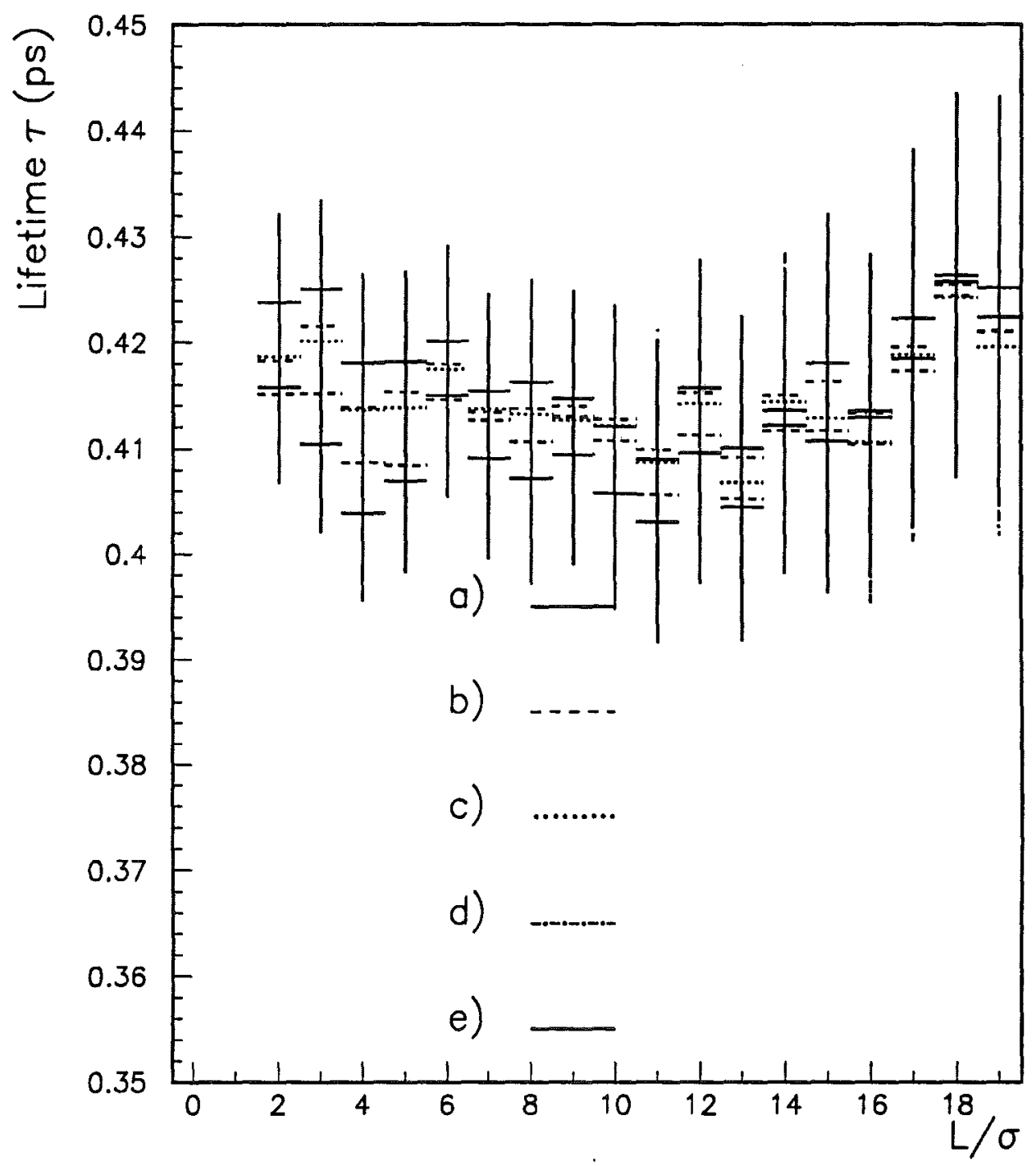

Figure 4.18. The $K \pi \pi \pi$ TAG sample lifetime as a function of $\ell / \sigma_{\ell}$ cut for the different fractions of sideband regions: a) low side band $100 \%$; b) low side band $75 \%$; c) low side band $50 \%$; d) low side band $25 \%$; e) low side band $0 \%$. 




Figure 4.19. The $K \pi \pi \pi$ NOTAG sample lifetime as a function of $\ell / \sigma_{\ell}$ cut for the different fractions of sideband regions: a) low side band $100 \%$; b) low side band $75 \%$; c) low side band $50 \%$; d) low side band $25 \%$; e) low side band $0 \%$. 

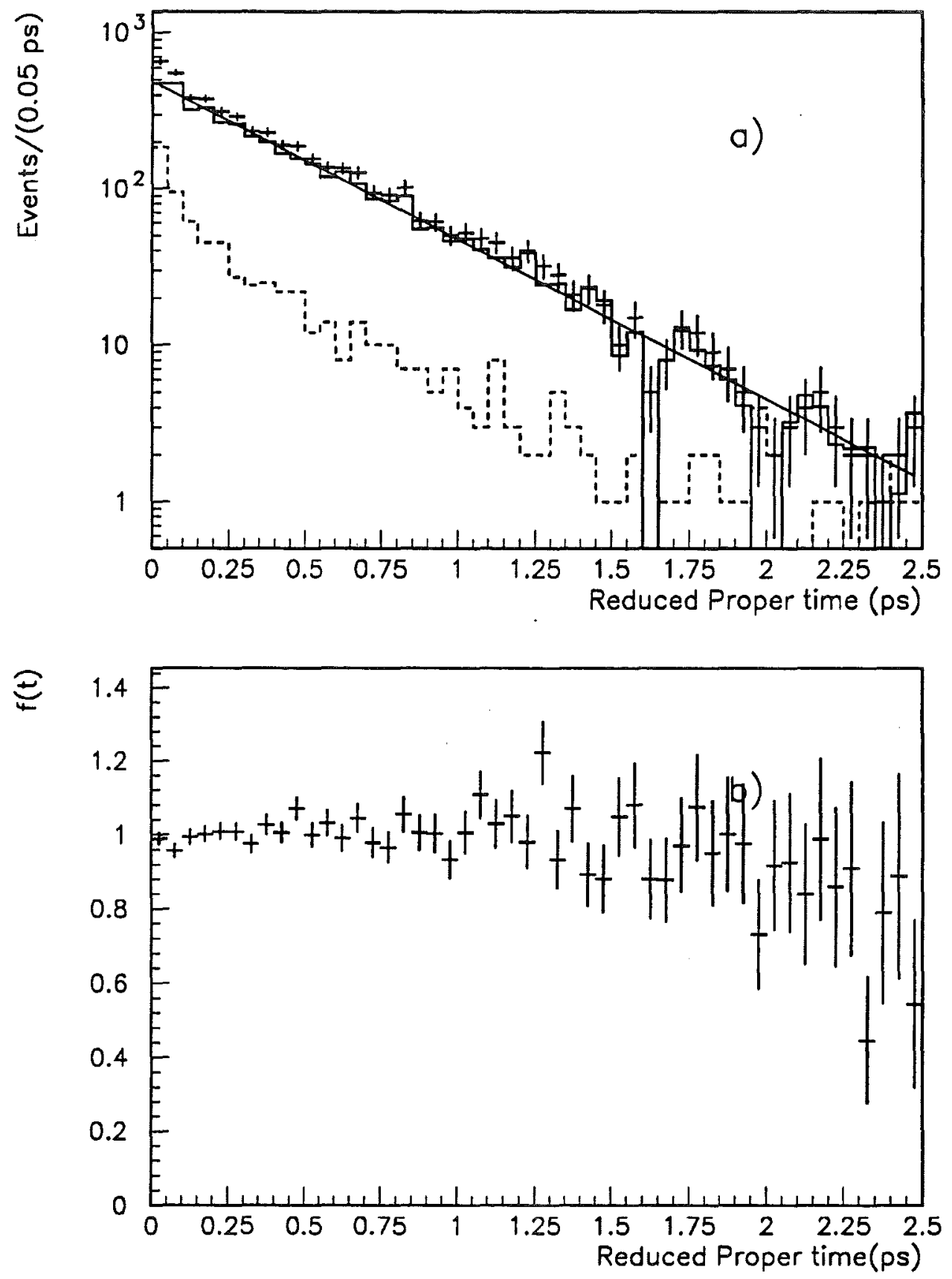

Figure 4.20, a) The background subtracted and $f\left(t^{\prime}\right)$ corrected reduced proper time distribution of the $K \pi$ TAG sample (shown as a solid histogram) for $\ell / \sigma_{\ell}>3$; crosses and dotted lines represent the signal and sideband reduced proper time distributions respectively. Superimposed is an exponential function with the corresponding fitted lifetime. b) $f\left(t^{\prime}\right)$ correction factor utilized in a). 

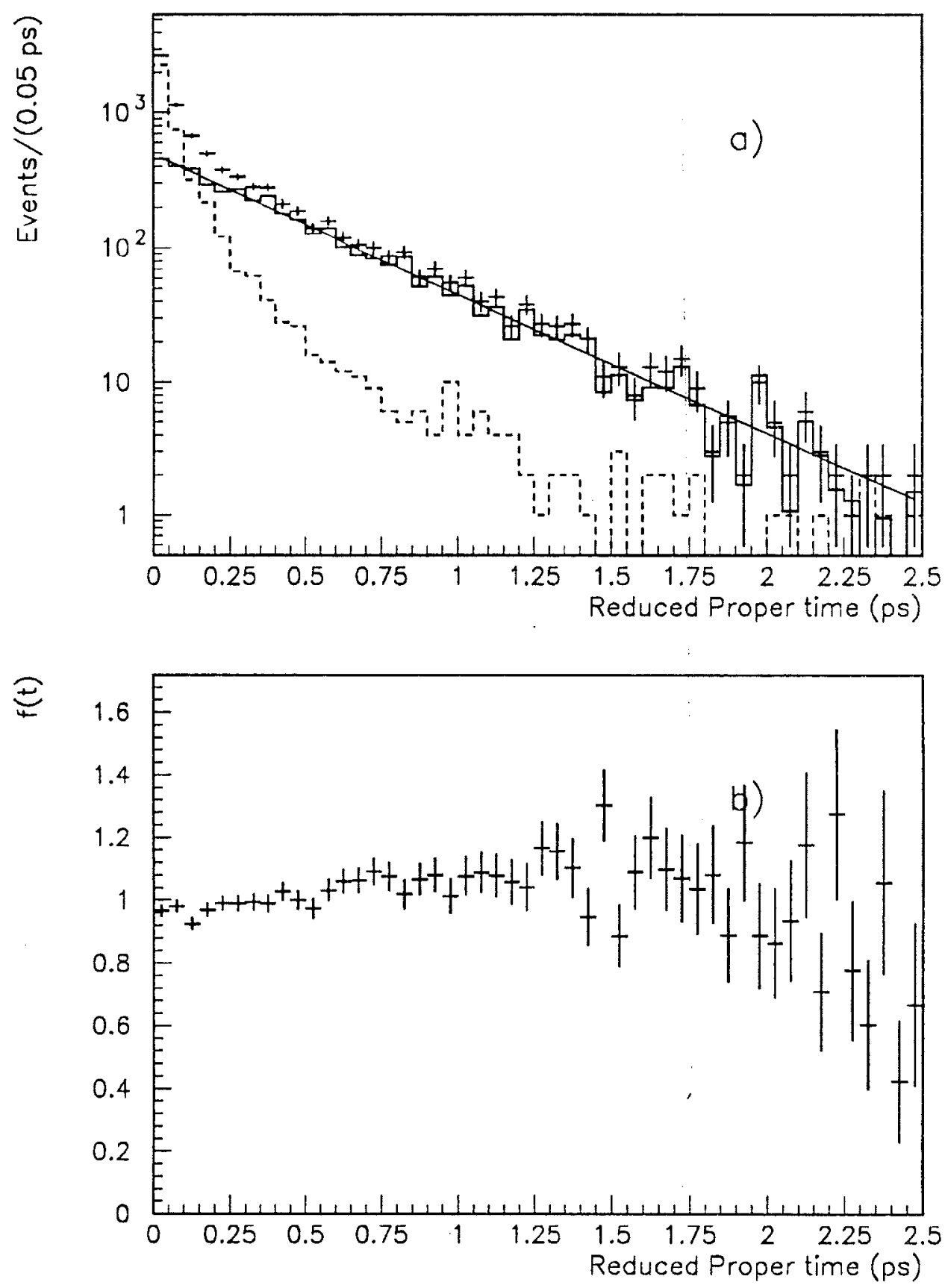

Figure 4.21. a) The background subtracted and $f\left(t^{\prime}\right)$ corrected reduced proper time distribution of the $K \pi \pi \pi$ TAG sample (shown as a solid histogram) for $\ell / \sigma_{\ell}>2$; crosses and dotted lines represent the signal and sideband reduced proper time distributions respectively. Superimposed is an exponential function with the corresponding fitted lifetime. b) $f\left(t^{\prime}\right)$ correction factor utilized in a). 

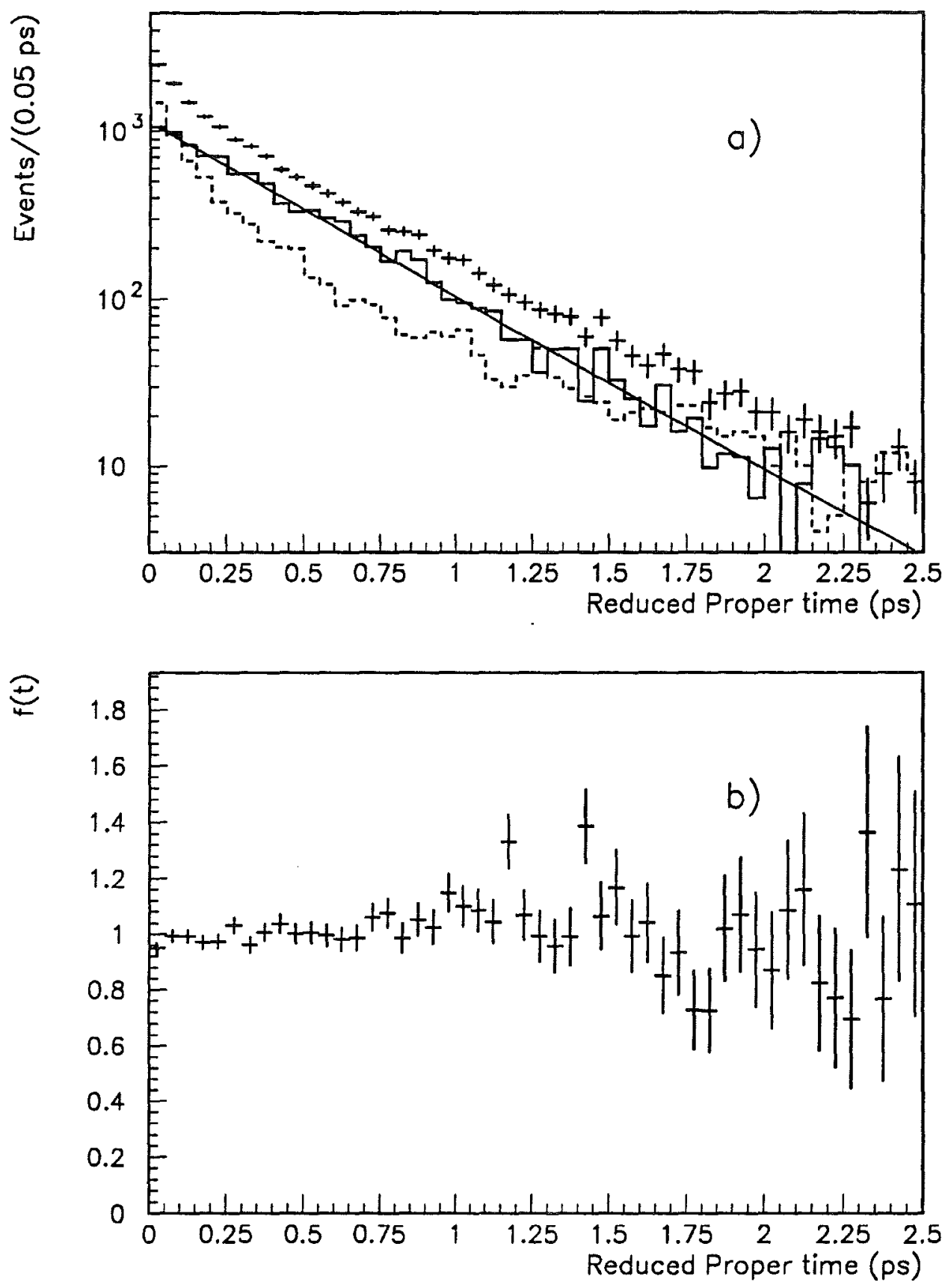

Figure 4.22. a) The background subtracted and $f\left(t^{\prime}\right)$ corrected reduced proper time distribution of the $K \pi \pi \pi$ NOTAG sample (shown as a solid histogram) for $\ell / \sigma_{\ell}>7$; crosses and dotted lines represent the signal and sideband reduced proper time distributions respectively. Superimposed is an exponential function with the corresponding fitted lifetime. b) $f\left(t^{\prime}\right)$ correction factor utilized in a). 

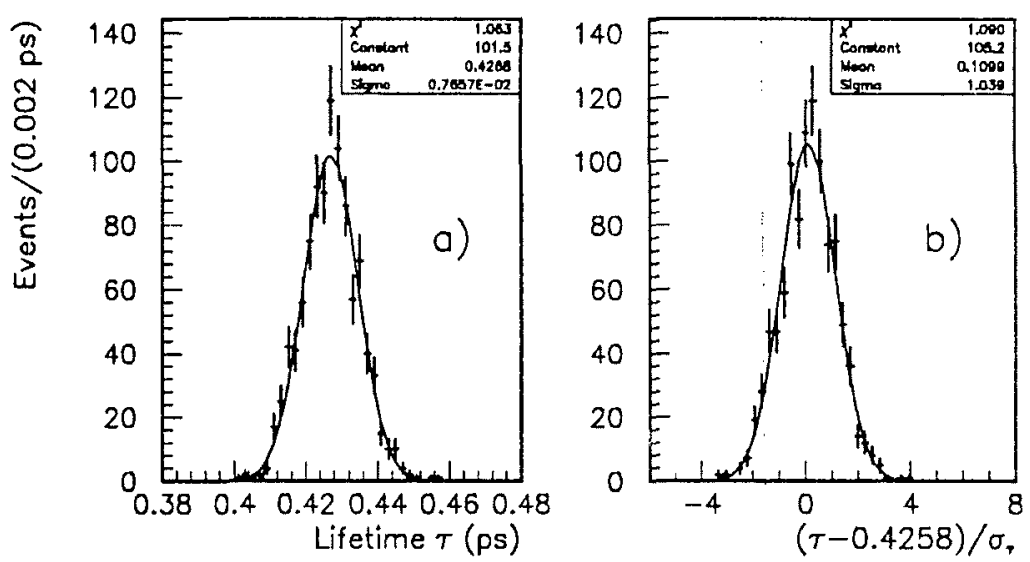

Figure 4.23. The result of simulated "mini-experiments" for the $K \pi$ TAG sample: a) distribution of the fitted lifetime for the $K \pi$ TAG sample; b) deviation of the fitted lifetime from the input value divided by its error for $K \pi$ TAG sample.

Table 4.4. Systematic Uncertainties of the $D^{0}$ Lifetime $\left(\times 10^{-12}\right.$ sec. $)$

\begin{tabular}{|c|c|c|c|}
\hline Source & $K \pi$ TAG & $K \pi \pi \pi$ TAG & $K \pi \pi \pi$ NOTAG \\
\hline Momentum & 0.0000 & 0.0000 & 0.0000 \\
Beam Profile & 0.0010 & 0.0010 & 0.0010 \\
Absorption & 0.0030 & 0.0030 & 0.0030 \\
Sidebands & 0.0020 & 0.0020 & 0.0020 \\
$f\left(t^{\prime}\right)$ & 0.0024 & 0.0027 & 0.0030 \\
\hline Total & 0.0044 & 0.0046 & 0.0048 \\
\hline
\end{tabular}

Table 4.5. The lifetimes of the $D^{0}$ samples

\begin{tabular}{|c|c|c|}
\hline Sample & $\ell / \sigma_{\ell}$ & Lifetime $\left(\times 10^{-12}\right)$ sec. \\
\hline$K \pi$ TAG & 3 & $0.4248 \pm 0.0077 \pm 0.0044$ \\
$K \pi \pi \pi$ TAG & 2 & $0.4190 \pm 0.0083 \pm 0.0046$ \\
$K \pi \pi \pi$ NOTAG & 7 & $0.4164 \pm 0.0075 \pm 0.0048$ \\
\hline
\end{tabular}



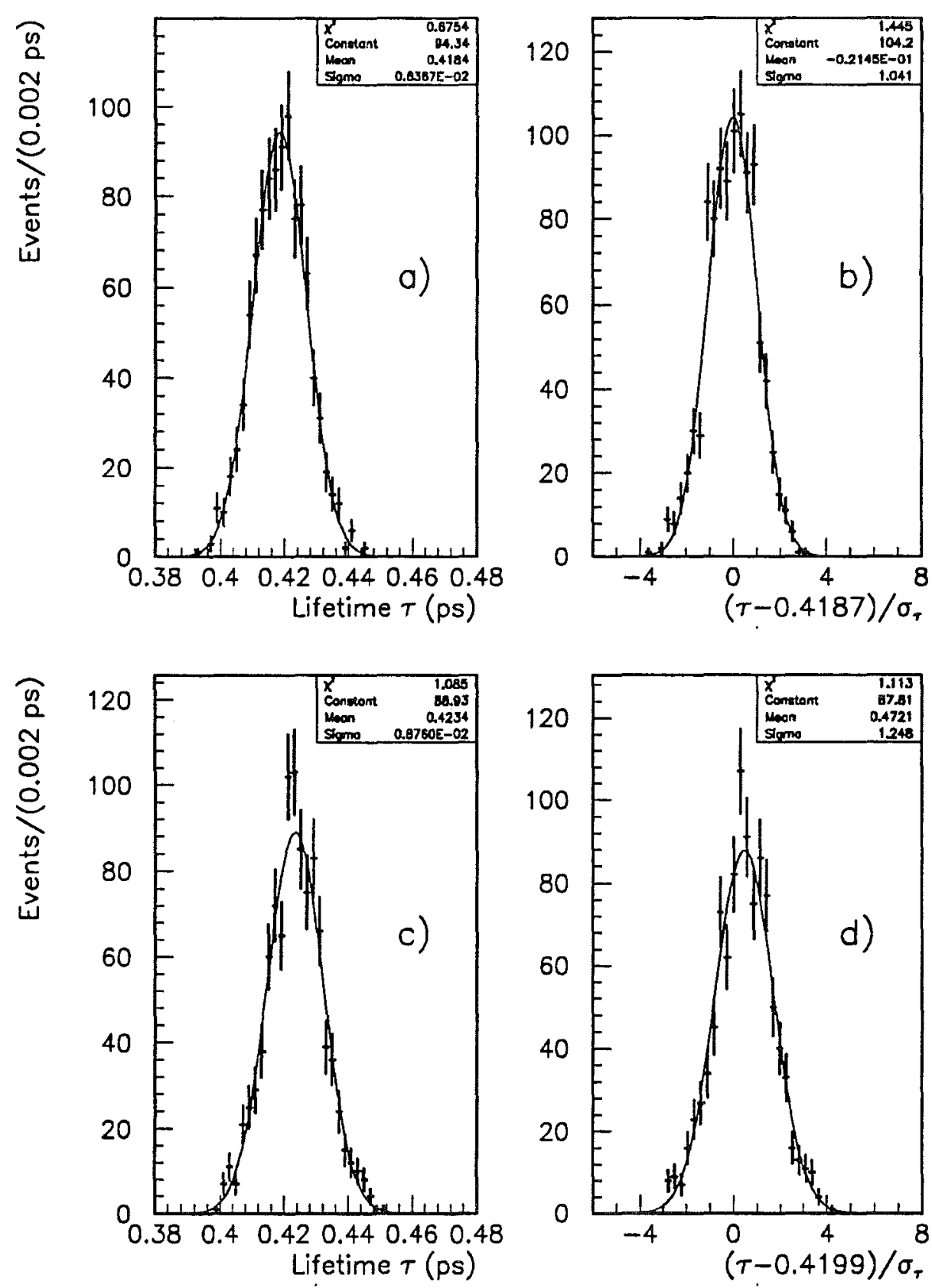

Figure 4.24. The result of simulated "mini-experiments" for $K \pi \pi \pi$ TAG and NOTAG samples: a) distribution of fitted lifetimes for the $K \pi \pi \pi$ TAG sample; b) deviation of the fitted lifetime from the input value divided by its error for $K \pi \pi \pi$ TAG sample; c) distribution of fitted lifetimes for the $K \pi \pi \pi$ NOTAG sample; d) deviation of the fitted lifetime the input value divided by its error for $K \pi \pi \pi$ NOTAG sample. 
measurements

$$
\tau\left(D^{0}\right)=\frac{1}{W} \sum_{i=1}^{i=3} w_{i} \tau_{i}
$$

where $w_{i}=1 / \sigma_{i}^{2}$ and $W=\sum w_{i}$. The variance of $\tau\left(D^{0}\right)$ is given by $1 / W$. In order to calculate the systematic error for the combined $D^{0}$ sample we assume that the errors associated with the beam profile and absorption are completely correlated, while the sideband systematic and $f\left(t^{\prime}\right)$ errors are completely uncorrelated between the samples. So the combined systematic error is given by

$$
<\delta \tau \delta \tau>=\frac{1}{W^{2}} \sum_{i, j} w_{i} w_{j}<\delta \tau_{i} \delta \tau_{j}>
$$

where

$$
\delta \tau_{i}=\delta \tau_{i}^{\mathrm{B}}+\delta_{i}^{\mathrm{A}}+\delta_{i}^{\mathrm{SB}}+\delta_{i}^{f\left(t^{\prime}\right)}
$$

and

$$
<\delta \tau_{i} \delta \tau_{j}>=<\delta \tau_{i}^{\mathrm{B}} \delta \tau_{j}^{\mathrm{B}}>+<\delta \tau_{i}^{\mathrm{A}} \delta \tau_{j}^{\mathrm{A}}>+<\delta_{i}^{\mathrm{SB}} \delta_{j}^{\mathrm{SB}}>_{!} \delta_{i j}+<\delta_{i}^{f\left(t^{\prime}\right)} \delta_{j}^{f\left(t^{\prime}\right)}>\delta_{i j}
$$

With this assumption we obtain 0.0030 ps for the systematic error of the combined $D^{0}$ sample.

In conclusion we have measured the $D^{0}$ lifetime to be

$$
\tau\left(D^{0}\right)=0.4200 \pm 0.0045 \pm 0.0030 \mathrm{ps}
$$


where the first error is statistical and second systematic. The confidence level on the hypothesis that all three measurements are consistent, using statistical errors is $>60 \%$. 


\section{$4.5 \quad D_{s}^{ \pm}$Lifetime}

The final $D_{s}^{ \pm}$sample consisted of 900 reconstructed decays (see Fig. 3.10). The $f\left(t^{\prime}\right)$ correction factor was derived from $500 \mathrm{~K}$ Monte Carlo events. The invariant mass distribution of the $\phi \pi$ sample includes, in addition to the $D_{s}^{ \pm}$peak a nearby peak corresponding to the Cabibbo suppressed decay of the $D^{ \pm} \rightarrow \phi^{0} \pi^{ \pm}$. Good invariant mass resolution of our detector enabled us to chose the lower mass sideband between the two peaks. Table 4.6 summarizes all the fit parameter values utilized in the fit. In Fig. 4.25 the fitted lifetime is plotted versus the $\ell / \sigma_{\ell}$ cut used. No significant variation of the fitted lifetime value is present with $\ell / \sigma_{\ell}$. The final value for the lifetime from this work is chosen to be the value at $\ell / \sigma_{\ell}>3$, which is in a region with a good signal-to-noise ratio and a small statistical error.

The value obtained for the lifetime is $\tau\left(D_{s}^{ \pm}\right)=0.475 \pm 0.020 \mathrm{ps}$. The background subtracted and Monte Carlo corrected reduced proper time distribution for the $D_{s}^{ \pm}$ signal is shown in Fig. 4.26 along with the raw reduced proper time distributions of the signal and sideband regions. Superimposed is a pure exponential function with the fitted value of the $D_{s}^{ \pm}$lifetime.

The distribution of the fitted lifetimes from two thousand simulated "mini-experiments" showed a presence of a small positive bias (overestimating the lifetime) of $0.002 \mathrm{ps}$,

Table 4.6. The $D_{s}^{ \pm}$Fit Parameters

\begin{tabular}{|c|c|}
\hline Parameter & Value \\
\hline Mean Mass & $1968.0 \pm 0.5 \mathrm{MeV} / c^{2}$ \\
Mass Resolution & $10.0 \pm 0.5 \mathrm{MeV} / c^{2}$ \\
Signal Mass Region & $1948.0-1988.0 \mathrm{MeV} / c^{2}$ \\
Low Sideband Mass Region & $1908.0-1928.0 \mathrm{MeV} / c^{2}$ \\
High Sideband Mass Region & $2008.0-2028.0 \mathrm{MeV} / c^{2}$ \\
$\ell / \sigma_{\ell}$ Requirement & $>3$ \\
Fitted Lifetime & $(0.475 \pm 0.020) \times 10^{-12}$ sec. \\
\hline
\end{tabular}



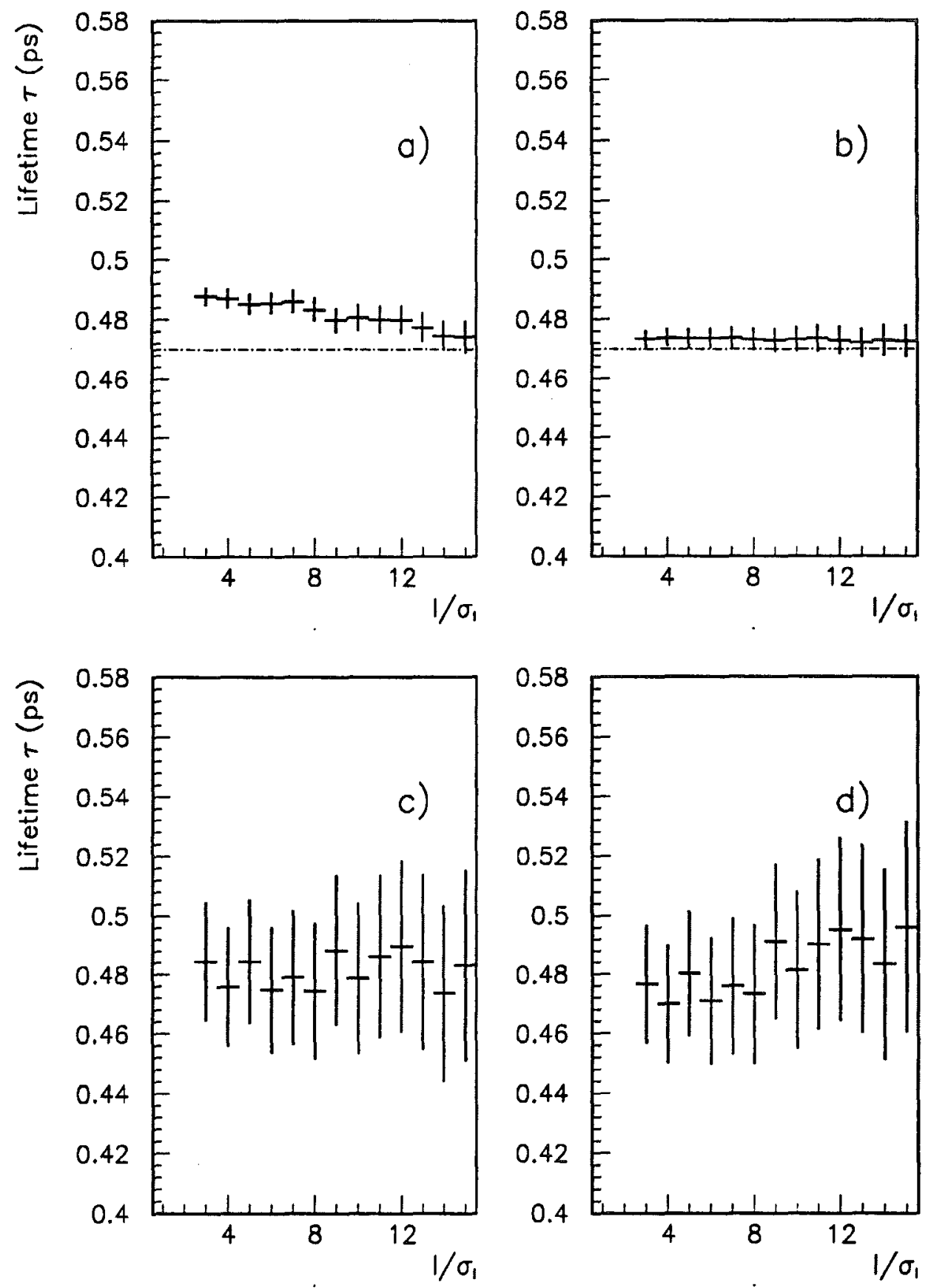

Figure 4.25. The lifetime of the $D_{s}^{ \pm}$as a function of $\ell / \sigma_{\ell}$ cut for: a) Monte Carlo sample with $f\left(t^{\prime}\right)=1$; b) data sample with $f\left(t^{\prime}\right)=1$; c) Monte Carlo sample with standard $f\left(t^{\prime}\right)$; d) data sample with standard $f\left(t^{\prime}\right)$. 


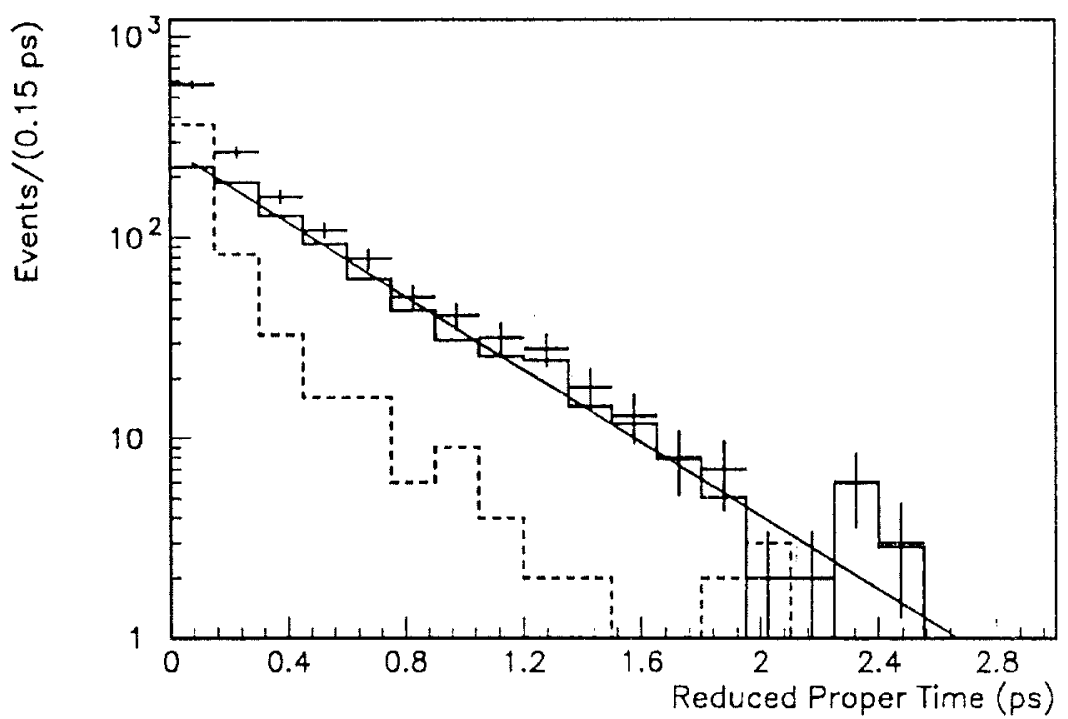

Figure 4.26. The background subtracted and Monte Carlo corrected lifetime evolution of the $D_{s}^{ \pm}$sample for $\ell / \sigma_{\ell}>3$ shown as the solid-lined histogram. The crosses show the reduced proper time distribution of the events in the signal region and the dotted-lined histogram shows the side band proper time distribution. The straight line is an exponential with fitted lifetime.

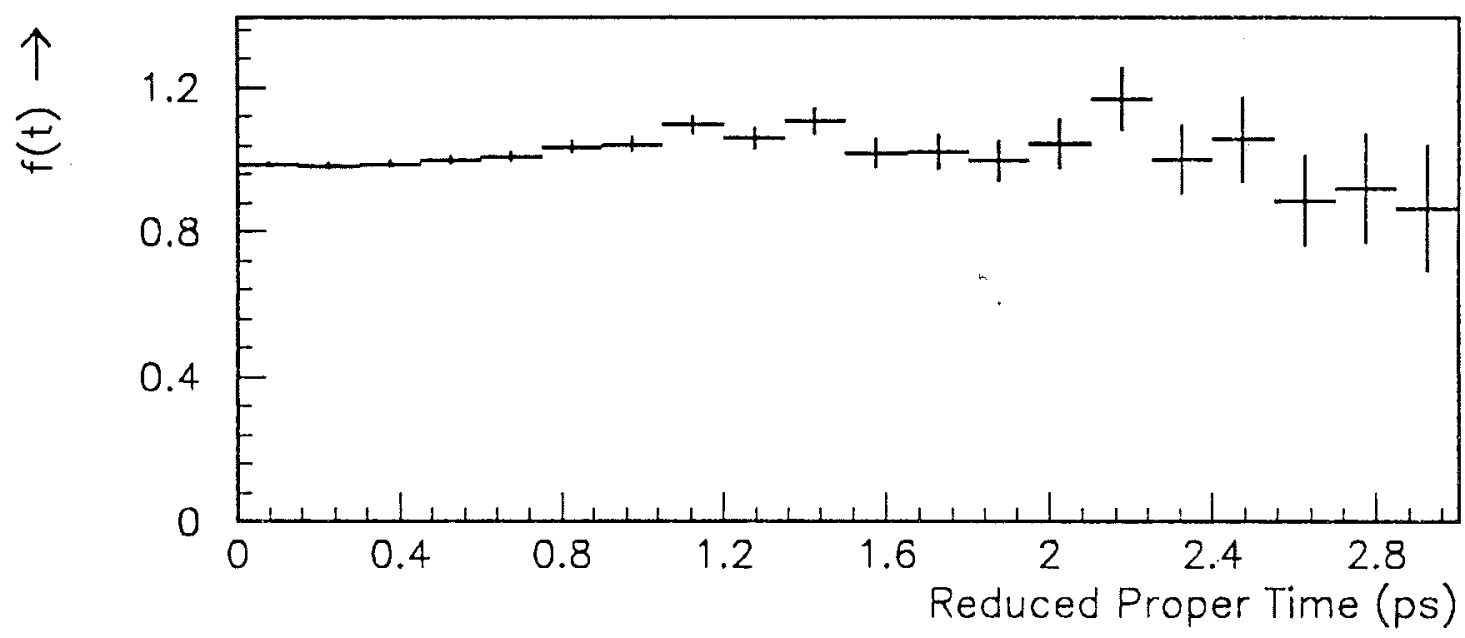

Figure 4.27. 'The correction factor $f\left(t^{\prime}\right)$ to the $D_{*}^{ \pm}$reduced proper time distribution as a function of reduced proper time for $\ell / \sigma_{\ell}>3$. 

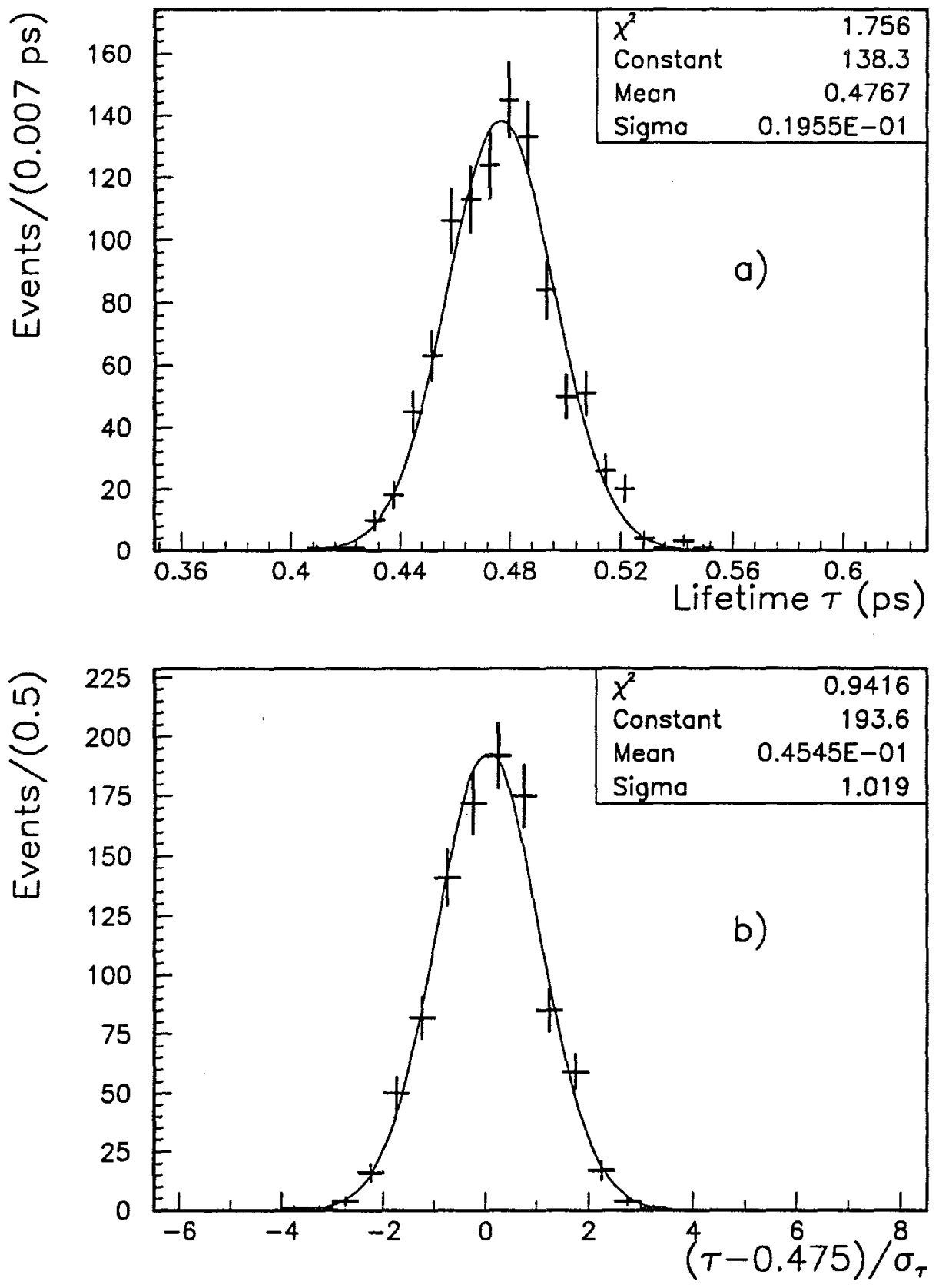

Figure 4.28. The results of fits to simulated "mini-experiments" for $D_{s}^{ \pm} \rightarrow \phi^{0} \pi^{ \pm}$sample: a) distribution of fitted lifetimes; $b$ ) deviation of the fitted lifetime from input value divided by its error. 


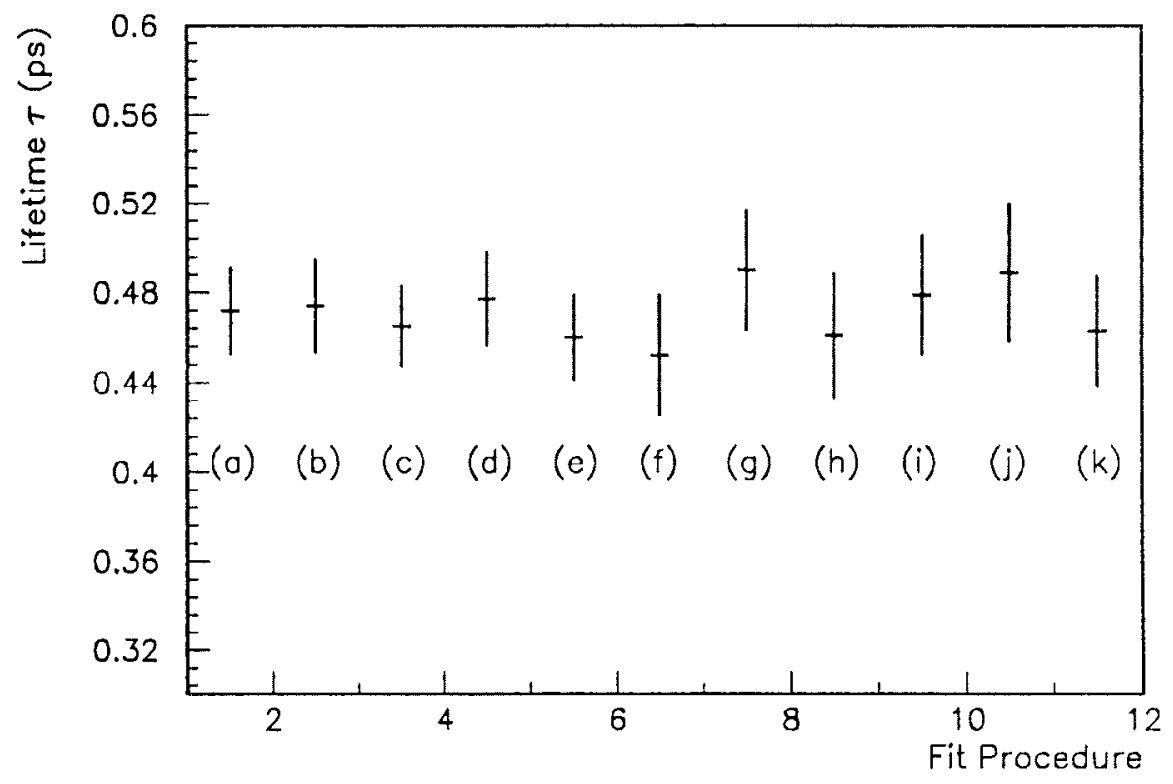

Figure 4.29. A comparison of measured $D_{s}$ lifetimes from different consistency checks and studies of systematic effects. The values are the results obtained: $a$ ) in this analysis; b) using side-band regions $1.788-1.808 \mathrm{GeV} / \mathrm{c}^{2}$ and $2.008-2.028 \mathrm{GeV} / \mathrm{c}^{2}$; c) using only side-band region $2.008-2.048 \mathrm{GeV} / \mathrm{c}^{2}$; d) eliminating events in the $D^{+}$reflection region; e) eliminating events in the $\Lambda_{c}$ reflection region; f) using only events with $D_{s}$ momentum $>95 \mathrm{GeV} / \mathrm{c} ; \mathrm{g}$ ) using only events with $D_{s}$ momentum $<95 \mathrm{GeV} / \mathrm{c} ; \mathrm{h}$ ) using $D_{s}^{+}$(particle) events only; i) using $D_{s}^{-}$(anti-particle) events only; j) using upstream primary vertices only, and $\mathbf{k}$ ) using downstream primary vertices only.

while the error calculated by the fitter proved to be accurate (see Fig. $4.28 \mathrm{~b}$ ). All quoted lifetime values have been corrected for this bias.

All the systematic studies showed that the variation of the fitted lifetime from model uncertainties were much smaller than the statistical error of the measurement. A comparison of measured lifetimes for the $D_{s}^{ \pm}$sample subject to several different constraints and partitions is shown in Fig. 4.29.

Table 4.7 summarizes the estimated systematic uncertainties. Combining all sources of systematic errors in quadrature, a systematic uncertainty of $0.007 \mathrm{ps}$ is ascribed to this measurement.

In summary the $D_{s}^{ \pm}$lifetime from 900 fully reconstructed $D_{s}^{ \pm} \rightarrow \phi^{0} \pi^{ \pm}$decays is 
Table 4.7. Systematic Uncertainties of the $D_{s}^{ \pm}$lifetime

\begin{tabular}{|c|c|}
\hline Source & Value $\left(\times 10^{-} 12\right)$ sec. \\
\hline$D^{ \pm}$Momentum & 0.0000 \\
Beam Profile & 0.0030 \\
Absorption & 0.0050 \\
Side Band & 0.0030 \\
$f\left(t^{\prime}\right)$ & 0.0026 \\
\hline Total & 0.0070 \\
\hline
\end{tabular}

measured to be

$$
\tau\left(D_{s}^{ \pm}\right)=0.475 \pm 0.020 \pm 0.007 \text { ps }
$$

where first error is statistical and second systematic. 


\section{CHAPTER 5}

\section{Summary and Conclusions}

This final chapter reviews the highlights of the E-687 experiment, and summarizes the performed measurements in this dissertation. The results are compared to other

high statistics measurements and the world average values for the $D^{0}, D^{ \pm}$and $D_{s}^{ \pm}$ lifetimes. The thesis is concluded with comments on these measurements and their possible implications.

The E-687 experiment was the successor to two generations of fixed target photoproduction experiments and as such benefited from the accumulated experience in detector design. The success of this experiment can be attributed to its excellent vertex measurement, particle identification and calorimetric capabilities, and a very dedicated crew ! It was in operation intermittently from June of 1987 to January 1992. The experiment used a high energy bremsstrahlung photon beam and a beryllium target. The average tagged photon momentum was $221 \mathrm{GeV} / \mathrm{c}$. During the $1990-92$ runs the experiment recorded $\approx 5 \times 10^{8}$ hadronic triggers and by the end of $1992 \mathrm{had} \approx 10^{5}$ reconstructed charm decays.

\subsection{Lifetime Measurements}

The measured lifetimes of the $D^{ \pm}, D^{0}$, and $D_{s}^{ \pm}$are summarized in Table 5.1. This table lists the obtained lifetimes with statistical and systematic uncertainties together with the sample sizes. 
Table 5.1. Summary of measured lifetimes

\begin{tabular}{|c|c|c|c|}
\hline Particle & Lifetime $\left(\times 10^{-} 12\right)$ sec. & Mode & No. of Events \\
\hline$D^{ \pm}$ & $1.042 \pm 0.015 \pm 0.011$ & $K^{\mp} \pi^{ \pm} \pi^{ \pm}$ & $\approx 11500$ \\
$D^{0}$ & $0.4200 \pm 0.0045 \pm 0.0030$ & $K^{\mp} \pi^{ \pm}$ & $\approx 4300$ \\
& & $K^{\mp} \pi^{ \pm} \pi^{\mp} \pi^{ \pm}$ & $\approx 13200$ \\
$D_{s}^{ \pm}$ & $0.475 \pm 0.020 \pm 0.007$ & $\phi^{0} \pi^{ \pm}$ & $\approx 900$ \\
\hline
\end{tabular}

The world average values [Pdg 92] for all three lifetimes are dominated by two previous measurements. These measurements were performed by E-691 Collaboration [Raa 88], and E-687 Collaboration [Cun 90b] [Cul 91b] from the data accumulated during the run in $1987-88$. Table 5.2 compares the values obtained in this thesis with the world averages and the two above mentioned measurements. Fig. 5.1 through Fig. 5.3 compare the lifetimes obtained in this thesis to all other lifetime measurements used by the particle data group to calculate the corresponding world average. The results are in agreement with the world average and the other two dominant measurements.

\subsection{Final Comments}

The sample of observed charm decays and the precision with which their lifetimes have been measured has dramatically improved over the last few years. This new sample from E-687 experiment will triple the world lifetime sample of $D^{ \pm}$and $D^{0}$ mesons and more than double the $D_{s}^{ \pm}$meson sample, hence significantly decreasing the uncertainties on these quantities. This measurement will tend to slightly lower the world average for the lifetime of the $D^{ \pm}$meson and increase the world average lifetime of the $D_{s}^{ \pm}$meson. From our measurements we obtain the following ratios:

$$
\frac{\tau\left(D^{ \pm}\right)}{\tau\left(D^{0}\right)}=2.49 \pm 0.04 \pm 0.03
$$




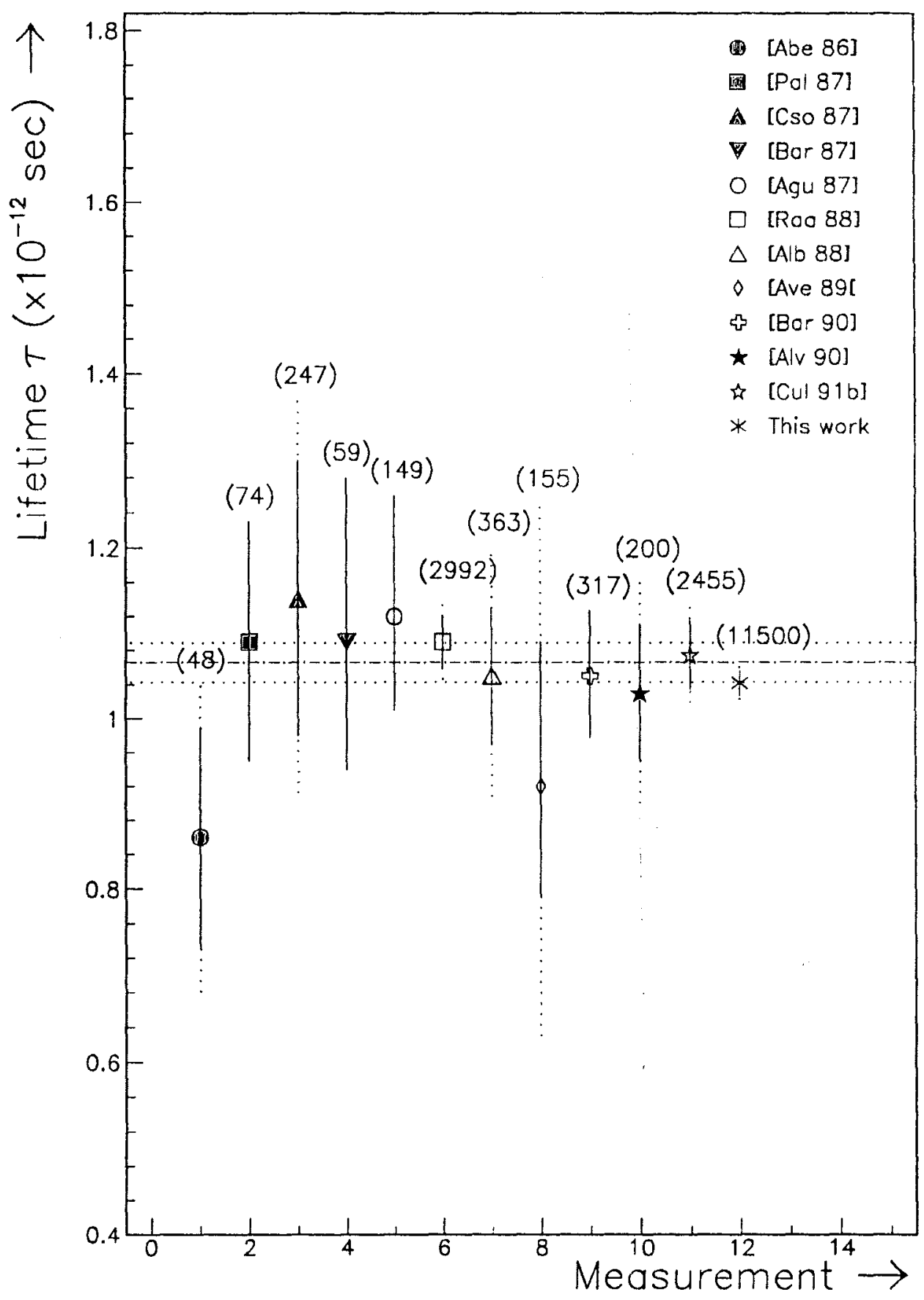

Figure 5.1. Comparison of the measured $D^{ \pm}$lifetime to other measurements used by the [Pdg 92] to calculate the previous worl average value shown as the horizontal dashed-dotted line. Vertical solid and dashed lines represent statistical and systematic errors respectively for a given measurement. Horizontal dotted line represents the error on the world average value. 


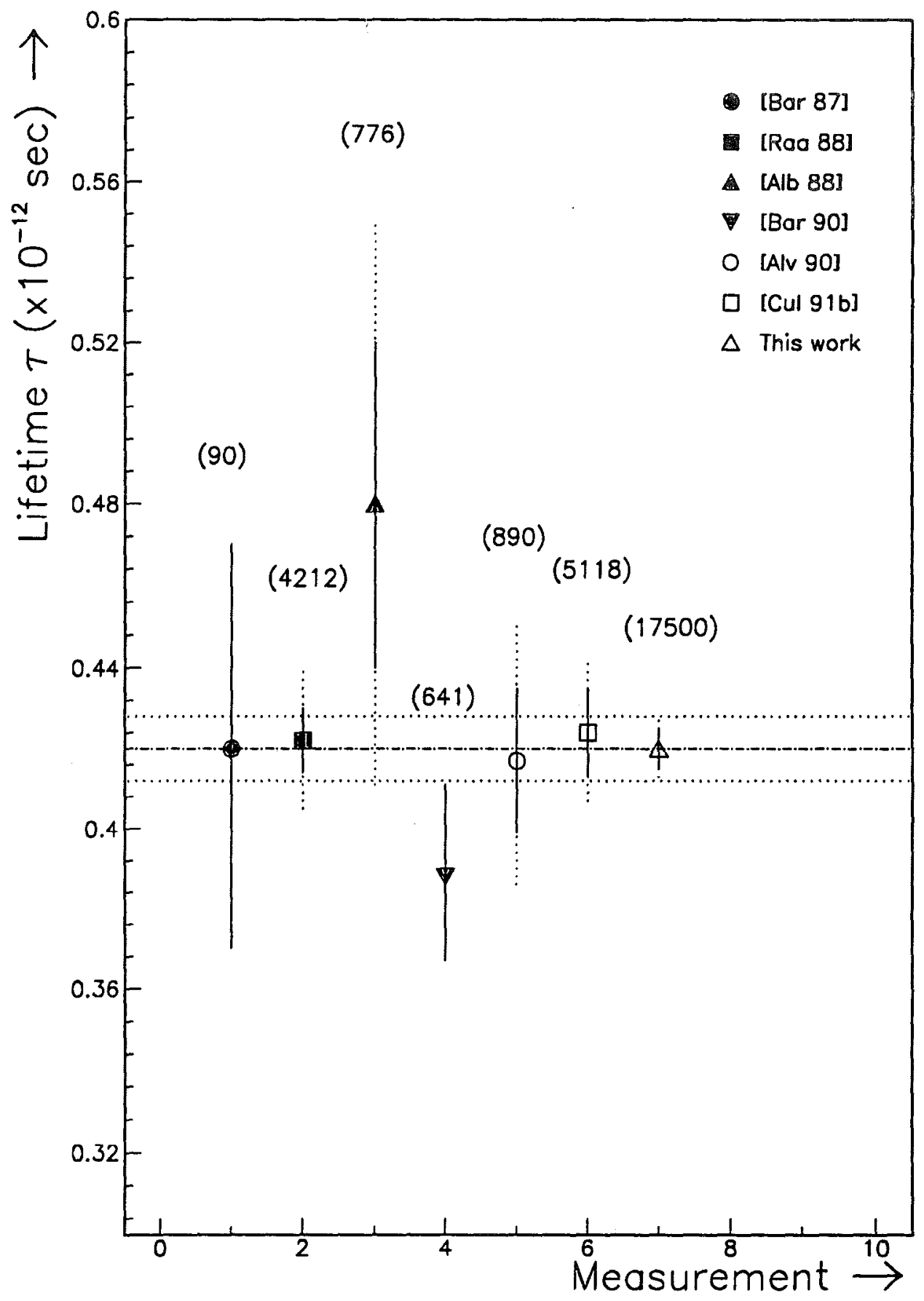

Figure 5.2. Comparison of the measured $D^{0}$ lifetime to other measurements used by the [Pdg 92] to calculate the previous worl average value shown as the horizontal dashed-dotted line. Vertical solid and dashed lines represent statistical and systematic errors respectively for a given measurement. Horizontal dotted line represents the error on the world average value. 


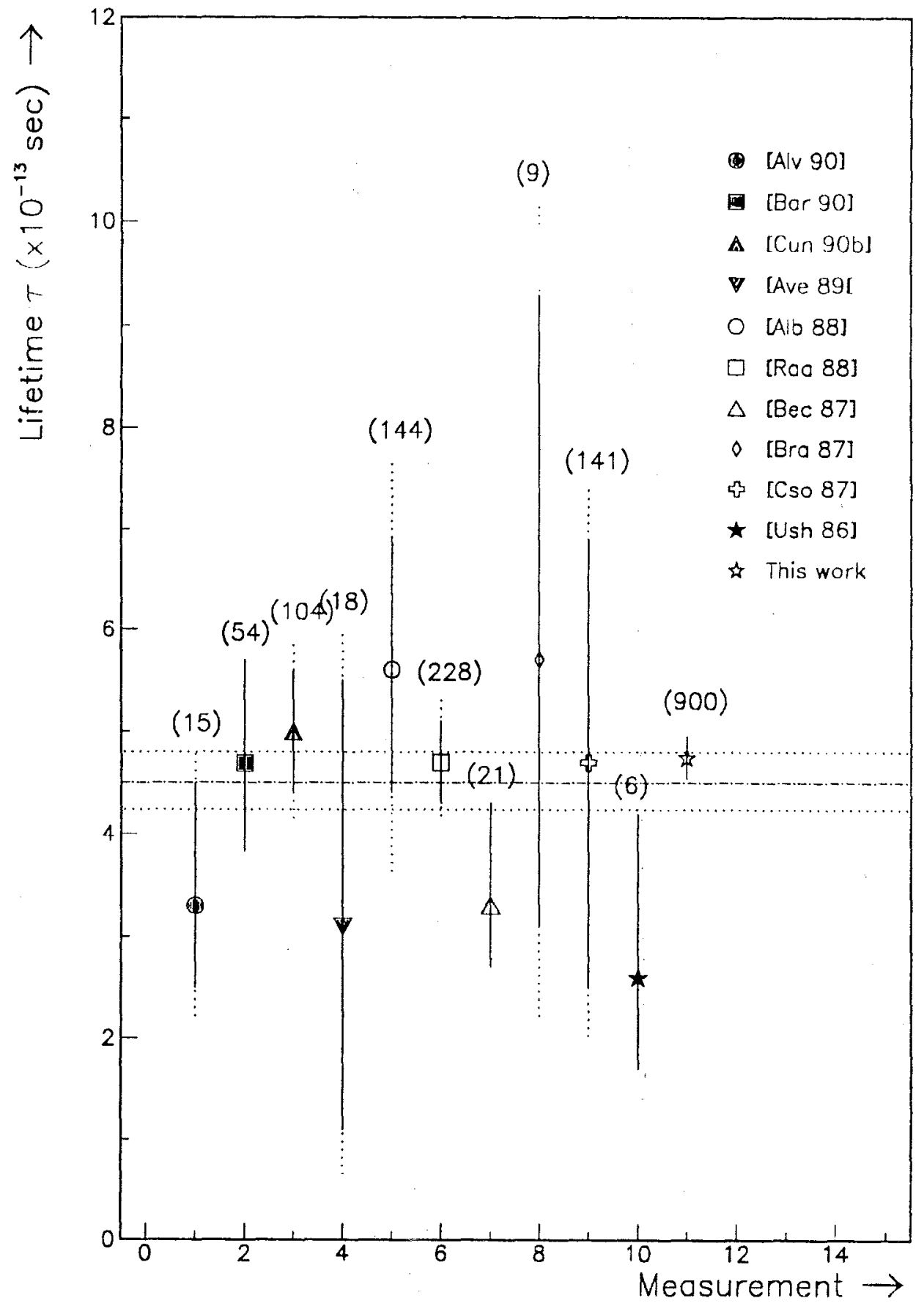

Figure 5.3. Comparison of the measured $D_{z}^{ \pm}$lifetime to other measurements used by the [Pdg 92] to calculate the previous worl average value shown as the horizontal dashed-dotted line. Vertical solid and dashed lines represent statistical and systematic errors respectively for a given measurement. Horizontal dotted line represents the error on the world average value. 
Table 5.2. Comparison of measured lifetimes

\begin{tabular}{|c|c|c|c|}
\hline Lifetime $\left(\times 10^{-} 12\right)$ sec. & No. of Events & Beam Type & Experiment \\
\hline \multicolumn{4}{|c|}{$D^{ \pm}$Lifetime } \\
\hline $1.066 \pm 0.023$ & World Average & & \\
\hline $1.075 \pm 0.040 \pm 0.018$ & 2455 & $\left.\gamma B e<\mathrm{E}_{\gamma}>\approx 220 \mathrm{GeV}\right)$ & $\mathbb{E}-687$ \\
\hline $1.090 \pm 0.030 \pm 0.025$ & 2992 & $\left.\gamma B e<\mathrm{E}_{\gamma}>\approx 145 \mathrm{GeV}\right)$ & E-691 \\
\hline $1.042 \pm 0.015 \pm 0.011$ & 11500 & $\left.\gamma B e<\mathrm{E}_{\gamma}>\approx 220 \mathrm{GeV}\right)$ & This Work \\
\hline \multicolumn{4}{|c|}{$\overline{D^{0} \text { Lifetime }}$} \\
\hline $0.420 \pm 0.008$ & World Average & & \\
\hline $0.424 \pm 0.011 \pm 0.007$ & 5118 & $\left.\gamma B e<\mathrm{E}_{\gamma}>\approx 220 \mathrm{GeV}\right)$ & E-687 \\
\hline $0.422 \pm 0.008 \pm 0.010$ & 4212 & $\left.\gamma B e<\mathrm{E}_{\gamma}>\approx 145 \mathrm{GeV}\right)$ & E-691 \\
\hline $0.4200 \pm 0.0045 \pm 0.0030$ & 17500 & $\left.\gamma B e<\mathrm{E}_{\gamma}>\approx 220 \mathrm{GeV}\right)$ & This Work \\
\hline \multicolumn{4}{|c|}{$D_{s}^{ \pm}$Lifetime } \\
\hline $0.450+0.030-0.026$ & World Average & & \\
\hline $0.500 \pm 0.060 \pm 0.030$ & 105 & $\gamma B e\left(<E_{\gamma}>\approx 220 \mathrm{GeV}\right)$ & E-687 \\
\hline $0.470 \pm 0.040 \pm 0.020$ & 228 & $\gamma B e\left(<\mathrm{E}_{\gamma}>\approx 145 \mathrm{GeV}\right)$ & E-691 \\
\hline $0.475 \pm 0.020 \pm 0.007$ & 900 & $\gamma B e\left(<\mathrm{E}_{\gamma}>\approx 220 \mathrm{GeV}\right)$ & This Work \\
\hline
\end{tabular}

and

$$
\frac{\tau\left(D_{s}^{ \pm}\right)}{\tau\left(D^{0}\right)}=1.13 \pm 0.05 \pm 0.02
$$

We conclude by noting that these measurements have significantly improved the precision with which the lifetimes of the $D^{ \pm}, D^{0}$ and $D_{s}^{ \pm}$are known. In fact the ratio of $D_{s}^{ \pm}$to $D^{0}$ lifetime has gained in significance from one to almost three sigma effect, but it is in the opposite direction from what one might expect and as such provides an exellent test for the theory [Big 92a] [Big 92b]. 


\section{BIBLIOGRAPHY}

[Abe 86] Abe et al., Phys. Rev. D 33, 1 (1986).

[Agu 87] Aguilar et al., Phys. Lett. B 193, 140 (1987).

[Alb 88] H.Albrecht et al., Phys. Lett. B 210, 267 (1988).

[Alv 90] M.P..Alvarez et al., Z.Phys. C 47, 539-545 (1990).

[Ave 89] D.Averill et al., Phys. Rev. D 39, 123 (1989).

[Bar 87] S.Barlag, et. al., Z.Phys. C 37, 17 (1987).

[Bar 90] S.Barlag, et. al., Z.Phys. C 46, 563-567 (1990).

[Bec 87] H.Becker, et. al., Phys. Lett. B 184, 277 (1987).

[Bel 86] G.Bellini et al., "The microstrip vertex detector for E687 experiment at Tevatron", Nucle. Instr. and Meth. in Phys. Res. A252, (1986) 366.

[Bel 92] G.Bellini et al., "A silicon active target for charm particle identification", Nucle. Instr. and Meth. in Phys. Res. A320, (1992) 439.

[Big 92a] I.I.Bigi and N.G.Uraltsev, Phys. Lett. B 280, 271-280 (1992).

[Big 92b] I.I.Bigi "A QCD manifesto on inclusive decays of beauty and charm', talk given at APS-DPF'92, November 1992. Phys. Lett. B 280, 271-280 (1992).

[Bil 84] N.Bilic,B.Guberina and J.Trampetic, Nucl. Phys. B2484, 261 (1984).

[Bra 87] W.Braunschweig, et. al., Z.Phys. C 35, 317-321 (1987).

[Cab 63] N.Cabibbo, Phys. Rev. Lett. 10, 531 (1963).

[Cha 84] ,L.L Chau and W.Y. Keung, Phys. Rev. Lett. 53, 1802 (1984). 
[Cso 87] S.E.Csorna, et. al., Phys. Lett. B 191, 318 (1987).

[Cul 91a] R.Culbertson,"Vertexing in the 90's", E687 internal memo, June 1991.

[Cul 91b] P.L. Frabetti, et. al., Phys. Lett. B 263, 584 (1991).

[Cun 90a] J.D.Cunningham,"A Measurement of the Lifetimes of $D_{s}^{+}$and $D^{+}$Mesons in High Energy Photoproduction", Ph.D. Thesis, University of Notre Dame (1990).

[Cun 90b] P.L. Frabetti, et. al., Phys. Lett. B 251, 639 (1990).

[Den 73] S.P.Denisov, et. al., Nucl. Phys. B 61, 62 (1973).

[Fra 92] P.L.Frabetti et al., "Description and performance of the Fermilab E687 spectrometer", Nucle. Instr. and Meth. in Phys. Res. A320, (1992) 519-547.

[Fra 93] P.L.Frabetti et al., "A wide band photon beam at the Fermilab Tevatron to study heavy flavours", to be submitted to Nucle. Instr. and Meth. in Phys. Res.

[Fro 79] A.G.Frodesen, O.Skjeggestad, and H.Tofte, "Probability and Statistics in Particle Physics", Univesitetsforlaget (1979).

[Gar 92] R.W.Gardner, "Lund Implementation in Rogue", E-687 internal memo (1992).

[Gub 79] B.Guberina,S.Nussinov,R.Peccei,R.Ruckl, Phys. Lett. 89B, 111 (1979).

[Inz 86] P.Inzani et al., "A fast ADC system for silicon microstrip readout", IEEE Transactions on Nuclear Science, Vol. NS-33, N.1, Feb. '86, pag. 911.

[Jam 89] F.James and M.Roos, "MINUIT Function Minimization and Error Analysis", CERN Computer Center Program Library, D506 (1989).

[Jar 90] G.Jarosse,"Skim a D Algortihm", E687 internal memo,1990

[Jdj 64] J.D.Jackson, Nuovo Cimento, Vol. XXXIV (1964).

[Jim 88] Jim Wiss, "Analytic Calculations Related to Micro Linking", E687 internal memo, August 1988.

[Jon 78] L.M.Jones and H.W.Wyld, Phys. Rev. D17, 759 (1978). 
[Kas 88] P.Kasper, et al., 'Wide Band to "Double Band", Upgrade, Fermilab, TM1552.

[Kob 73] M.Kobayashi and T.Maskawa, Prog. Theor. Phys. 49, 652 (1973).

[Lin 90] K.Lingel, "Photoproduction of $D^{ \pm}$mesons", Ph.D. Thesis, University of Illinois, (1990).

[Mor 88] L.Moroni and D.Pedrini, "All you always wanted to know about Microrico but were afraid to ask", E687 internal memo,November 1988.

[Mou 92] R.Mountain, "Photon and Hadron Inteactions in the Scintillating Fiber Target" Ph.D. Thesis, University of Notre Dame 1992.

[Pal 87] Palka et al., Z.Phys. C 35, 151 (1987).

[Pdg 92] Particle Data Group, Review of Particle Properties, Phys. Rev. D 45, Part 2 (1992).

[Pet 90] D.Petravick, et al., "The PAN-DA Data Acquisition System", Fermilab preprint, FERMILAB-Conf-89/136.

[Raa 88] J.R.Raab, et. al., Phys. Rev. D 37, 2391 (1988).

[Ren 90] P.Renton,'Electroweak Interactions', Cambridge University Press, (1990).

[Ros 80] S.P.Rosen, Phys. Rev. Lett. 44, 4 (1980).

[Ruck 83] R.Ruchl,"Weak Decays of Heavy Flavours", Habilitationsschrift submitted to the University of Munich.

[Ste 87] Sten Hansen, Fermilab Physics Department, drawing numbers 3687-EC240543, 3687-EC-240544.

[Ush 86] N.Ushida et al., Phys. Rev. Lett. 56, 1771 (1986). 
